JOURNAL OF THE

AMERICAN MATHEMATICAL SOCIETY

Volume 11, Number 4, October 1998, Pages 899-965

S $0894-0347(98) 00277-\mathrm{X}$

\title{
REGULARITY OF THE FREE BOUNDARY FOR THE POROUS MEDIUM EQUATION
}

\author{
P. DASKALOPOULOS AND R. HAMILTON
}

\section{INTRODUCTION}

We consider the Cauchy problem for the porous medium equation

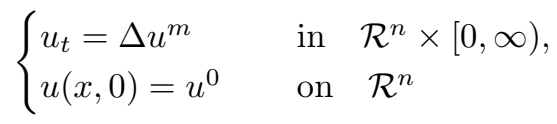

in the range of exponents $m>1$, with initial data $u^{0}$ nonnegative, integrable and compactly supported.

It is well known that this equation describes the evolution in time of various diffusion processes, in particular the flow of a gas through a porous medium; $u$ represents the density, while $f=m u^{m-1}$ represents the pressure of the gas. The function $f$ satisfies the equation

$$
f_{t}=f \Delta f+r(m)|D f|^{2}
$$

with $r(m)=1 /(m-1)$. When $u=0$, then $f=0$ and both of the above equations become degenerate. This degeneracy results in the interesting phenomenon of the finite speed of propagation: If the initial data $u^{0}$ is compactly supported in $\mathcal{R}^{n}$, the solution $u(\cdot, t)$ will remain compactly supported for all time $t$.

In this work we will show that, under rather general assumptions on the initial data, the free boundary

$$
\Gamma=\partial \overline{\operatorname{supp} u}
$$

is a smooth surface when $0<t<T$, for some $T>0$.

It is well known that if the initial data $u^{0}$ is nonnegative, integrable and compactly supported, then the Cauchy problem for the porous medium equation admits a unique solution on $\mathcal{R}^{n} \times(0, \infty)$ which has constant mass. However, since the equation becomes degenerate when $u=0$, the solution is not expected to be smooth; the optimal regularity for the density $u$ has been shown to be Hölder continuous and solutions are understood in the distributional sense. On the other hand, the physical interpretation of the equation indicates that, under ideal conditions, the free boundary should be a smooth surface and the pressure $f$ a smooth function up to the interface. However, this is not always the case: If support of the initial data $u^{0}$ is topologically complex, then advancing free-boundaries may hit each other after a short time, creating singularities.

Received by the editors January 19, 1998.

1991 Mathematics Subject Classification. Primary 35Jxx.

Key words and phrases. Porous medium equation, free-boundary, $C^{\infty}$-regularity.

(C) 1998 American Mathematical Society 
Caffarelli and Friedman ([CF1], [CF2]) showed that the interface can always be described by a Hölder continuous function $t=S(x), x \notin \operatorname{supp} u_{0}$, for any initial data. In the one-dimensional case much more is known: It has been shown in $[\mathrm{K}]$ and $[\mathrm{CF} 1]$ that if the support of the initial data is an interval, the free boundary consists of two Lipshitz continuous curves $x=\zeta_{i}(t)$, with $\zeta_{1}$ decreasing and $\zeta_{2}$ increasing. Moreover, there exist waiting times $t_{i}^{*}$ so that $\zeta_{i}(t)$ are constant for $t \leq t_{i}^{*}$ and each $\zeta_{i}$ is $C^{1}$ for $t>t_{i}^{*}$. However at $t=t_{i}^{*}, \zeta_{i}(t)$ may have a corner, so Lipshitz is the optimal regularity (see $[\mathrm{ACV}])$. Aronson and Vázquez $([\mathrm{AV}])$ and independently Höllig and Kreiss ([HK]) showed that for $t>t_{i}^{*}$ the curves $\zeta_{i}$ are smooth. Angenent ([A]) showed that for $t>t_{i}^{*}$ the curves $\zeta_{i}$ are real analytic.

In dimensions $n \geq 2$ the regularity of the free boundary poses a much harder question. Caffarelli, Vázquez and Wolanski ([CVW]) showed that, under the assumption that at time $t=0$ the pressure $f^{0} \in C^{1}\left(\overline{\operatorname{supp} f^{0}}\right)$ and $D f^{0} \neq 0$ along $\partial \overline{\operatorname{supp} f^{0}}$, a condition which ensures that the free boundary will start to move at each point at $t=0$, the interface can be described by a Lipshitz continuous function $t=S(x), x \notin \operatorname{supp} f^{0}$. Caffarelli and Wolanski ([CW]) improved this result, showing that, under the same hypotheses, the free boundary is a $C^{1, \alpha}$ surface. However, it is not shown in $[\mathrm{CW}]$ that the pressure $f$ is a $C^{1, \alpha}$ function up to the free boundary.

In this work, assuming that the initial pressure $f^{0}$ is strictly positive in the interior of a compact domain $\Omega$ in $\mathcal{R}^{2}$, with $f^{0}=0$ on $\partial \Omega \cup\left(\mathcal{R}^{n} \backslash \Omega\right)$, and denoting by $d$ the distance to the boundary of $\Omega$, we obtain the following result:

Theorem $\left(C^{\infty}\right.$-regularity of the boundary). If the functions $f^{0}, D f^{0}$ and $d D^{2} f^{0}$, restricted to the compact domain $\Omega$, extend continuously up to the boundary $\Omega$, with extensions which are Hölder continuous on $\Omega$ of class $C^{\alpha}(\Omega)$, for some $\alpha>0$, and $D f^{0} \neq 0$ along $\partial \Omega$, then there exists a number $T>0$ for which the initial value problem

$$
\begin{cases}f_{t}=f \Delta f+r|D f|^{2}, & (x, t) \in \mathcal{R}^{n} \times[0, T], \\ f(x, 0)=f^{0}, & x \in \mathcal{R}^{n},\end{cases}
$$

admits a solution $f$ which is smooth up to the interface $\Gamma$, when $0<t<T$. In particular, the free boundary $\Gamma$ is a smooth surface, when $0<t<T$.

The paper is divided into three parts: In Part 1 we study the model linear degenerate equation

$$
f_{t}=x\left(f_{x x}+f_{y y}\right)+\nu f_{x}+g
$$

with $\nu>0$ on the half-space $x \geq 0$, and no extra conditions on $f$ along the boundary $x=0$. The diffusion in this equation is governed by the Riemannian metric $d s^{2}=\left(d x^{2}+d y^{2}\right) / 2 x$. It is a simple observation that the equation admits solutions which behave like $x^{1-\nu}$ near the boundary $x=0$. However, once these solutions are ruled out, all other solutions are smooth. The basic idea in our approach is to establish Schauder type coercive estimates for the solutions $f$ of the model equation, which are scaled according to the new metric $d s$. In Part 2 we extend the results of Part 1 to a certain class of quasilinear degenerate evolution equations. The proof of the regularity of the free boundary is given in Part 3. Using a global change of coordinates, we transform the free boundary problem to a fixed boundary problem for a degenerate quasilinear equation, which can be solved, in appropriately defined Hölder spaces, using the results from Parts 1 and 2. 
Part I. The MOdel Linear DEgenerate Equation

I.1. The results. The first part of the paper will be devoted to the study of the model degenerate equation

$$
f_{t}=x\left(f_{x x}+f_{y y}\right)+\nu f_{x}+g
$$

with $\nu>0$ on the half-space $x \geq 0$, and no extra conditions on $f$ along the boundary $x=0$. The diffusion is governed by the Riemannian metric $d s$ where

$$
d s^{2}=\frac{d x^{2}+d y^{2}}{2 x} .
$$

We call this the cycloidal metric because its geodesics are cycloid curves. Its Laplace operator is

$$
\Delta_{s} f=2 x\left(f_{x x}+f_{y y}\right) .
$$

Our evolution equation can be written with respect to the new metric as

$$
f_{t}=\frac{1}{2} \Delta_{s} f+\nu f_{x}
$$

which is diffusion in the cycloidal metric with transport velocity $\nu$. The distance between two points $\left(\begin{array}{l}x_{1} \\ y_{1}\end{array}\right)$ and $\left(\begin{array}{l}x_{2} \\ y_{2}\end{array}\right)$ in this metric is a function

$$
s\left[\left(\begin{array}{l}
x_{1} \\
y_{1}
\end{array}\right),\left(\begin{array}{l}
x_{2} \\
y_{2}
\end{array}\right)\right]
$$

which is equivalent to the function

$$
\bar{s}\left[\left(\begin{array}{l}
x_{1} \\
y_{1}
\end{array}\right),\left(\begin{array}{l}
x_{2} \\
y_{2}
\end{array}\right)\right]=\frac{\left|x_{1}-x_{2}\right|+\left|y_{1}-y_{2}\right|}{\sqrt{x_{1}}+\sqrt{x_{2}}+\sqrt{\left|y_{1}-y_{2}\right|}}
$$

in the sense that

$$
s \leq C \bar{s} \quad \text { and } \quad \bar{s} \leq C s
$$

for some constant $C$. For the parabolic problem we use the parabolic distance

$$
s\left[\left(\begin{array}{l}
x_{1} \\
y_{1} \\
t_{1}
\end{array}\right),\left(\begin{array}{l}
x_{2} \\
y_{2} \\
t_{2}
\end{array}\right)\right]=s\left[\left(\begin{array}{l}
x_{1} \\
y_{1}
\end{array}\right),\left(\begin{array}{l}
x_{2} \\
y_{2}
\end{array}\right)\right]+\sqrt{\left|t_{1}-t_{2}\right|} .
$$

In terms of this distance we can define Hölder continuity. We say a continuous function $g$ on a compact subset $\mathcal{A}$ of the half-space $\{(x, y, t): x \geq 0\}$ is Hölder continuous with respect to the metric $s$ if for all points $P_{1}$ and $P_{2}$ in $\mathcal{A}$ we have

$$
\left|g\left(P_{1}\right)-g\left(P_{2}\right)\right| \leq C s\left[P_{1}, P_{2}\right]^{\alpha}
$$

and we define the Hölder semi-norm

$$
\|g\|_{H_{s}^{\alpha}(\mathcal{A})}=\sup _{P_{1} \neq P_{2}}\left|g\left(P_{1}\right)-g\left(P_{2}\right)\right| / s\left[P_{1}, P_{2}\right]^{\alpha} .
$$

We also define the norm

$$
\|g\|_{C_{s}^{\alpha}(\mathcal{A})}=\|g\|_{\mathcal{C}^{0}(\mathcal{A})}+\|g\|_{H_{s}^{\alpha}(\mathcal{A})}
$$

where as usual

$$
\|g\|_{C^{0}(\mathcal{A})}=\sup _{P}|g(P)| .
$$

With this norm the space $\mathcal{C}_{s}^{\alpha}(\mathcal{A})$ of Hölder continuous functions on $\mathcal{A}$ with respect to the metric $s$ is a Banach space (as usual). 
Now suppose the set $\mathcal{A}$ is the closure of its interior, and the function $f$ on $\mathcal{A}$ has continuous derivatives

$$
f_{t}, f_{x}, f_{y}, f_{x x}, f_{x y}, f_{y y}
$$

in the interior of $\mathcal{A}$, and that

$$
f_{t}, f_{x}, f_{y} \quad \text { and } \quad x f_{x x}, x f_{x y}, x f_{y y}
$$

extend continuously to the boundary, and the extensions are Hölder continuous on $\mathcal{A}$ of class $\mathcal{C}_{s}^{\alpha}(\mathcal{A})$ as before. Let $\mathcal{C}_{s}^{2+\alpha}(\mathcal{A})$ be the Banach space of all such functions with norm

$$
\begin{aligned}
\|f\|_{\mathcal{C}_{s}^{2+\alpha}(\mathcal{A})} & =\|f\|_{C_{s}^{\alpha}(\mathcal{A})}+\left\|f_{x}\right\|_{C_{s}^{\alpha}(\mathcal{A})}+\left\|f_{y}\right\|_{C_{s}^{\alpha}(\mathcal{A})}+\left\|f_{t}\right\|_{C_{s}^{\alpha}(\mathcal{A})} \\
& +\left\|x f_{x x}\right\|_{C_{s}^{\alpha}(\mathcal{A})}+\left\|x f_{x y}\right\|_{C_{s}^{\alpha}(\mathcal{A})}+\left\|x f_{y y}\right\|_{C_{s}^{\alpha}(\mathcal{A})}
\end{aligned}
$$

The operator $L_{0}$ defined by

$$
L_{0}=f_{t}-x\left(f_{x x}+f_{y y}\right)-\nu f_{x}
$$

defines a continuous linear map

$$
L_{0}: C_{s}^{2+\alpha}(\mathcal{A}) \rightarrow C_{s}^{\alpha}(\mathcal{A}) .
$$

We can extend these definitions to spaces of higher order derivatives. Let $k$ be a positive integer and let $\mathcal{A}$ be a subset of the half-space $x \geq 0$ as above. We denote by $C_{s}^{k, \alpha}(\mathcal{A})$ the space of all functions $g$ whose $k$-th order derivatives $D_{x}^{i} D_{y}^{j} D_{t}^{l} g$, $i+j+l=k$, exist and belong to the space $C_{s}^{\alpha}(\mathcal{A})$. Similarly, we denote by $C_{s}^{k, 2+\alpha}(\mathcal{A})$ the space of all functions $f$ on $\mathcal{A}$ whose $k$-th order derivatives $D_{x}^{i} D_{y}^{j} D_{t}^{l} f$, $i+j+l=k$, exist and belong to the space $C_{s}^{2+\alpha}(\mathcal{A})$, as defined above. Both spaces, equipped with the norms

$$
\|g\|_{C_{s}^{k, \alpha}(\mathcal{A})}=\sum_{i+j+l \leq k}\left\|D_{x}^{i} D_{y}^{j} D_{t}^{l} g\right\|_{C_{s}^{\alpha}(\mathcal{A})}
$$

and

$$
\|f\|_{C_{s}^{k, 2+\alpha}(\mathcal{A})}=\sum_{i+j+l \leq k}\left\|D_{x}^{i} D_{y}^{j} D_{t}^{l} f\right\|_{C_{s}^{2+\alpha}(\mathcal{A})}
$$

are Banach spaces. The last norm is equivalent to the norm

$$
\|f\|_{C_{s}^{k, 2+\alpha}(\mathcal{A})}=\sum_{i+j+l \leq k+1}\left\|D_{x}^{i} D_{y}^{j} D_{t}^{l} f\right\|_{C_{s}^{\alpha}(\mathcal{A})}+\sum_{\substack{i+j+l=k+2 \\ l \leq k}}\left\|x D_{x}^{i} D_{y}^{j} D_{t}^{l} f\right\|_{C_{s}^{\alpha}(\mathcal{A})} .
$$

We denote by $C_{s}^{0, \alpha}(\mathcal{A})$ and $C_{s}^{0,2+\alpha}(\mathcal{A})$ the spaces $C_{s}^{\alpha}(\mathcal{A})$ and $C_{s}^{2+\alpha}(\mathcal{A})$ respectively. The operator

$$
L_{0}: C_{s}^{k, 2+\alpha}(\mathcal{A}) \rightarrow C_{s}^{k, \alpha}(\mathcal{A})
$$

defines a continuous linear map.

Denoting by $\mathcal{S}_{0}$ the half-space $x \geq 0$ in $\mathcal{R}^{2}$, by $\mathcal{S}$ the space $\mathcal{S}=\mathcal{S}_{0} \times[0, \infty)$, and by $\mathcal{S}_{T}$ the space $\mathcal{S}_{0} \times[0, T]$, for $T>0$, we state now the main result of Part I:

I.1.1. Theorem (Existence and Uniqueness). Let $k$ be a nonnegative integer and let $\alpha$ be a number in $0<\alpha<1$. Assume that $g \in \mathcal{C}_{s}^{k, \alpha}(\mathcal{S})$ and $f^{0} \in \mathcal{C}_{s}^{k, 2+\alpha}\left(\mathcal{S}_{0}\right)$, 
with both $g$ and $f^{0}$ compactly suported in $\mathcal{S}$ and $\mathcal{S}_{0}$ respectively. Then, for any $\nu>0$ and $T>0$, the initial value problem

$$
\begin{cases}L_{0} f=g & \text { in } S_{T}, \\ f(\cdot, 0)=f^{0} & \text { on } S_{0}\end{cases}
$$

admits a unique solution $f \in \mathcal{C}_{s}^{k, 2+\alpha}\left(\mathcal{S}_{T}\right)$. Moreover

$$
\|f\|_{\mathcal{C}_{s}^{k, 2+\alpha}\left(\mathcal{S}_{T}\right)} \leq C(T)\left(\left\|f^{0}\right\|_{\mathcal{C}_{s}^{k, 2+\alpha}\left(\mathcal{S}_{0}\right)}+\|g\|_{\mathcal{C}_{s}^{k, \alpha}(\mathcal{S})}\right)
$$

for some constant $C(T)$ depending only $\alpha, k, \nu$ and $T$.

Theorem I.1.1 follows from the following two results:

I.1.2. Theorem (Existence and Uniqueness of Smooth Solutions). Assume that $g$ is a smooth function with compact support on $\mathcal{S}=\mathcal{S}_{0} \times[0, \infty)$, which vanishes at $t=0$. Then, for any $\nu>0$, there exists a unique smooth solution $f$ of the initial value problem

$$
\begin{cases}L_{0} f=g & \text { in } S, \\ f(\cdot, 0)=0 & \text { on } S_{0} .\end{cases}
$$

Moreover, for any $T>0$ there exists a constant $C(T)$ depending only on $\nu$ and $T$ so that

$$
\|f\|_{C^{0}\left(\mathcal{S}_{T}\right)} \leq C(T)\|g\|_{C^{0}(\mathcal{S})} .
$$

Define the box of side $r$ around a point $P=\left(\begin{array}{l}x_{0} \\ y_{0} \\ t_{0}\end{array}\right)$ to be

$$
\mathcal{B}_{r}(P)=\left\{\left(\begin{array}{l}
x \\
y \\
t
\end{array}\right): \begin{array}{c}
x \geq 0,\left|x-x_{0}\right| \leq r \\
t_{0}-r \leq t \leq t_{0} \mid \leq r
\end{array}\right\} .
$$

We let $\mathcal{B}_{r}$ be the box around the point $P=\left(\begin{array}{l}0 \\ 0 \\ 1\end{array}\right)$.

I.1.3. Theorem (Schauder Estimate). Let $k$ be a nonnegative integer and let $0<$ $\alpha<1$ and $\nu>0$. Then, for any $r<1$ there exists a constant $C$ depending on $k$, $\alpha, \nu$ and $r$ so that

$$
\|f\|_{C_{s}^{k, 2+\alpha}\left(\mathcal{B}_{r}\right)} \leq C\left(\|f\|_{C_{s}^{\circ}\left(\mathcal{B}_{1}\right)}+\left\|L_{0} f\right\|_{C_{s}^{k, \alpha}\left(\mathcal{B}_{1}\right)}\right)
$$

for all $C^{\infty}$ smooth functions $f$ on $\mathcal{B}_{1}$.

I.2. The metric. We begin by elaborating on the metric

$$
d s^{2}=\frac{d x^{2}+d y^{2}}{2 x} .
$$

The nonzero components of $g_{i j}$ where

$$
d s^{2}=g_{i j} d x^{i} d x^{j}
$$

are

$$
g_{x x}=g_{y y}=\frac{1}{2 x} .
$$

The nonzero components of the inverse $g^{i j}$ are

$$
g^{x x}=g^{y y}=2 x .
$$


The area element $a=\mu d x d y$ with

$$
\mu=\sqrt{\operatorname{det} g_{i j}}
$$

is given by

$$
\mu=\frac{1}{2 x}
$$

The Laplace operator in general is

$$
\Delta_{s} f=\frac{1}{\mu} \frac{\partial}{\partial x^{i}}\left(\mu g^{i j} \frac{\partial}{\partial x^{j}} f\right)
$$

and in our case this gives

$$
\Delta_{s} f=2 x\left(f_{x x}+f_{y y}\right) .
$$

The Christoffel symbols are given in general by

$$
\Gamma_{i j}^{\ell}=\frac{1}{2} g^{k \ell}\left(\frac{\partial}{\partial x^{i}} g_{j k}+\frac{\partial}{\partial x^{j}} g_{i k}-\frac{\partial}{\partial x^{k}} g_{i j}\right)
$$

and in our case the nonzero Christoffel symbols are

$$
\Gamma_{x x}^{x}=-\frac{1}{2 x}, \quad \Gamma_{y y}^{x}=+\frac{1}{2 x}, \quad \Gamma_{x y}^{y}=-\frac{1}{2 x} .
$$

The shortest distance between two points is a geodesic, which in general satisfies the equations

$$
\frac{d^{2} x^{k}}{d s^{2}}+\Gamma_{i j}^{k} \frac{d x^{i}}{d s} \frac{d x^{j}}{d s}=0
$$

where $s$ is the arc length; in our case we get the system

$$
\left\{\begin{array}{l}
\frac{d^{2} x}{d s^{2}}-\frac{1}{2 x}\left(\frac{d x}{d s}\right)^{2}+\frac{1}{2 x}\left(\frac{d y}{d s}\right)^{2}=0 \\
\frac{d^{2} y}{d s^{2}}-\frac{1}{x} \frac{d x}{d s} \frac{d y}{d s}=0 .
\end{array}\right.
$$

A curve parametrized by arc length has speed one, so

$$
\frac{1}{2 x}\left[\left(\frac{d x}{d s}\right)^{2}+\left(\frac{d y}{d s}\right)^{2}\right]=1 .
$$

We can solve for $d y / d s$ in terms of $d x / d s$ and substitute in the equation for $d^{2} x / d s^{2}$ to get an equation independent of $y$; then substituting $v=d x / d s$ reduces this to a first order equation which is separable after the substitution $v=x p$. This allows us to solve explicitly for the geodesics.

I.2.1. Proposition. The cycloid curve

$$
\left\{\begin{array}{c}
x=1-\cos s \\
y=s-\sin s
\end{array}\right.
$$

is a geodesic parametrized by arc length $s$ for the metric

$$
d s^{2}=\frac{d x^{2}+d y^{2}}{2 x}
$$

and all the other geodesics are obtained by translation $y \rightarrow y+b$ and dilation $x \rightarrow c x$, $y \rightarrow c y$, or are horizontal lines. 
Proof. The reader can verify that the cycloid solves the equations for a geodesic by substitution. Translations leave the metric fixed, while dilations expand it by a fixed factor, so the translates and dilates are also geodesics. Since there is one such curve through each point with $x>0$ in each direction, this is all of them. To see this, pick the point on the cycloid arch for $0<s<2 \pi$ with the desired slope, and then translate and dilate it till it reaches the desired point. This works because the slope is invariant under translation and dilation. The only exception is that the horizontal slope is attained only at the ends $s=0$ or $2 \pi$. This corresponds to horizontal lines, which are also solutions of the geodesic equations, and correspond to limits of dilations $x \rightarrow c x, y \rightarrow c y$ as $c \rightarrow \infty$.

Note that the boundary curve $x=0$ is at a finite distance from the interior. Along the horizontal lines $d y=0$ and

$$
d s=\frac{d x}{\sqrt{2 x}}
$$

so

$$
s=\sqrt{2 x}
$$

exactly; while along the catenoid

$$
x=1-\cos s=\frac{s^{2}}{2}-\frac{s^{4}}{24}+\ldots
$$

so

$$
s=\sqrt{2 x}\left(1+\frac{x}{24}+\ldots\right)
$$

and both are finite. By contrast along a vertical line $d x=0$ and

$$
d s=\frac{d y}{\sqrt{2 x}}
$$

so the length of a vertical segment from $\left(\begin{array}{l}x_{0} \\ y_{1}\end{array}\right)$ to $\left(\begin{array}{l}x_{0} \\ y_{2}\end{array}\right)$ goes to infinity as $x_{0} \rightarrow 0$. When $x_{0}$ is very small compared to $\left|y_{1}-y_{2}\right|$ the shortest path is along a catenoid which at first plunges fearlessly into the interior nearly horizontally at first until $x$ is comparable to $\left|y_{1}-y_{2}\right|$, then moves vertically up where vertical distances are much shorter, and finally returns toward the boundary nearly horizontally. This motivates our understanding of our Hölder norms; near the boundary the action is essentially always horizontal. Diffusion over a distance $s$ in a time $t$ takes place on scales where $s^{2} \approx t$. Near the boundary most of the diffusion is horizontal on scales where $x \approx s^{2}$ so $x \approx t$. Here the drift from the term $\nu f_{x}$ becomes comparable to the diffusion. We need $\nu>0$ so that the drift is toward the boundary; if we were in the case $\nu<0$ we would need a boundary condition.

In Section I.2 we will prove the existence and uniqueness of Theorem I.1.2. Sections I.4-I.9 will be devoted to the proof of the Schauder estimates in Theorem I.1.3. In Section I.10 we will prove an extension lemma for Hölder spaces with respect to the metric $s$. The proof of Theorem I.1.1 will be given in Section I.12.

I.3. Proof of Theorem I.1.2. Assume that $g$ is a smooth function with compact support on $\mathcal{S}=\mathcal{S}_{0} \times[0, \infty)$, with $\mathcal{S}_{0}$ denoting, as before, the half-space $x \geq 0$ in 
$\mathcal{R}^{2}$, which vanishes at $t=0$. We will show the existence of a smooth solution of the initial value problem

$$
\begin{cases}L_{0} f=g & \text { in } S \\ f(\cdot, 0)=0 & \text { on } S_{0}\end{cases}
$$

with

$$
L_{0} f=f_{t}-x\left(f_{x x}+f_{y y}\right)-\nu f_{x}
$$

such that

$$
\|f\|_{C^{0}(\mathcal{S})} \leq C\|g\|_{C^{0}(\mathcal{S})}
$$

for some absolute constant $C$. For this purpose, we convert the equation $L_{0} f=g$ to an ordinary differential equation in $x$ with a regular singular point at $x=0$, by applying the Fourier-Laplace transform in the variables $(y, t), t \geq 0$. Indeed, it is easy to see that $f$ is a solution of the equation

$$
f_{t}-x\left(f_{x x}+f_{y y}\right)-\nu f_{x}=g
$$

if and only if its Fourier-Laplace transform in $(y, t)$, defined as

$$
\widetilde{f}(x, \xi, \tau)=\int_{t=0}^{\infty} e^{-t \tau} d t \int_{y=-\infty}^{\infty} f(x, y, t) e^{-i y \xi} d y
$$

for $\xi$ a real number and $\tau$ a complex number with $\operatorname{Re}(\tau)>0$, satisfies the ordinary differential equation

$$
x \widetilde{f}_{x x}+\nu \widetilde{f}_{x}-\left(x \xi^{2}+\tau\right) \widetilde{f}=-\widetilde{g}
$$

with

$$
\widetilde{g}(x, \xi, \tau)=\int_{t=0}^{\infty} e^{-t \tau} d t \int_{y=-\infty}^{\infty} g(x, y, t) e^{-i y \xi} d y .
$$

Since $g$ is smooth and compactly supported in $S$, it is standard to show that the function $\widetilde{f}$ is well defined, smooth and decays rapidly as $|\xi| \rightarrow \infty$ and $|\tau| \rightarrow \infty$ with $\operatorname{Re}(\tau)>0$, while it is compactly supported in $x \in[0, \infty)$. The point $x=0$ is a regular singular point for the ordinary differential equation satisfied by $\widetilde{f}$. Hence, for all real $\xi$ and complex $\tau$ with $\operatorname{Re}(\tau)>0$, the equation has a unique smooth solution $\widetilde{f}(x, \xi, \tau)$ on the half-line $x \geq 0$. Moreover, the solution $\widetilde{f}$ will depend smoothly on the parameters $\xi$ and $\tau$, making $\widetilde{f}$ a smooth function with respect to all variables $(x, \xi, \tau), \operatorname{Re}(\tau)>0$. We will show that for every $x \geq 0$, the function $\widetilde{f}(x, \xi, \tau)$ decays rapidly as $|\xi| \rightarrow \infty,|\tau| \rightarrow+\infty, \operatorname{Re}(\tau)>0$, and hence its inverse Fourier-Laplace transform

$$
f(x, y, t)=\lim _{\epsilon \rightarrow 0} \int_{-i \infty+\epsilon}^{+i \infty+\epsilon} e^{t \tau} d \tau \int_{\xi=-\infty}^{+\infty} \widetilde{f}(x, \xi, \tau) e^{i y \xi} d \xi
$$

is well defined on $\mathcal{S}$, is smooth and is nothing but the desired solution of the equation $L_{0} f=g$.

Indeed, if we write $\widetilde{f}=p+i q, \widetilde{g}=h+i k$ and $x \xi^{2}+\tau=\rho+i \sigma$, the ordinary differential equation satisfied by $\widetilde{f}$ becomes equivalent to the system

$$
\left\{\begin{array}{l}
x p_{x x}+\nu p_{x}-\rho p+\sigma q=h \\
x q_{x x}+\nu q_{x}-\rho q-\sigma p=k
\end{array}\right.
$$


Then $F=\left(p^{2}+q^{2}\right) / 2$ satisfies the differential inequality

$$
x F_{x x}+\nu F_{x}-2 \rho F \geq p h+q k .
$$

By Young's inequality we have

$$
p h+q k \geq-\rho\left(\frac{p^{2}+q^{2}}{2}\right)-\frac{C}{\rho}\left(h^{2}+k^{2}\right) \geq-\rho F-\frac{C}{\rho} A(\xi, \tau)
$$

with $A(\xi, \tau)=\sup _{x>0}\left(h^{2}+k^{2}\right)(x, \xi, \tau)$. Hence, since $\rho=x \xi^{2}+\operatorname{Re}(\tau) \geq \operatorname{Re}(\tau)$ the function

$$
\widetilde{F}=F-\frac{C}{(\operatorname{Re}(\tau))^{2}} A
$$

satisfies the differential inequality

$$
x \widetilde{F}_{x x}+\nu \widetilde{F}_{x}-\operatorname{Re}(\tau) \widetilde{F} \geq 0 .
$$

Moreover $\widetilde{F}$ is smooth and bounded on $x \geq 0$, for all real $\xi$ and complex $\tau$ with $\operatorname{Re}(\tau)>0$. It follows from the maximum principle that $\widetilde{F} \leq 0$, for all $x \geq 0$ which gives us the bound

$$
|\widetilde{f}|(x, \xi, \tau) \leq \frac{C}{R e(\tau)} \sup _{x \geq 0}|\widetilde{g}|(x, \xi, \tau)
$$

with $C$ an absolute, positive constant. Since $\sup _{x \geq 0}|\widetilde{g}|(x, \xi, \tau)$ decays rapidly as $|\xi| \rightarrow \infty,|\tau| \rightarrow \infty$ with $\operatorname{Re}(\tau)>0$, it follows from this estimate that the function $f$ given by

$$
f(x, y, t)=\lim _{\epsilon \rightarrow 0} \int_{-i \infty+\epsilon}^{+i \infty+\epsilon} e^{t \tau} d \tau \int_{\xi=-\infty}^{+\infty} \tilde{f}(x, \xi, \tau) e^{i y \xi} d \xi
$$

is well defined and therefore a smooth solution of the equation $L_{0} f=g$. It is also easy to see that

$$
\|f\|_{C^{0}\left(\mathcal{S}_{T}\right)}<\infty
$$

for all $T>0$. In addition, for any positive integer $n$, we have

$$
D_{t}^{n} f(x, y, t)=\lim _{\epsilon \rightarrow 0} \int_{-i \infty+\epsilon}^{+i \infty+\epsilon} e^{t \tau} d \tau \int_{\xi=-\infty}^{+\infty} \tau^{n} \tilde{f}(x, \xi, \tau) e^{i y \xi} d \xi .
$$

Therefore, denoting by $\mathcal{L}$ the Laplace transform, we have

$$
\mathcal{L}\left(D_{t}^{n} f\right)(x, y, \tau)=\tau^{n} \mathcal{L}(f)(x, y, \tau)
$$

for all $\tau>0$. This immediately implies that

$$
D_{t}^{n} f(x, y, 0)=0
$$

for all positive integers $n$, making $f$ a smooth function on $\mathcal{S}$ with $f(\cdot, \cdot, 0)=0$. This answers the existence question. The uniqueness of bounded smooth solutions as well as the estimate $\|f\|_{C^{0}(\mathcal{S})} \leq C\|g\|_{C^{0}(\mathcal{S})}$ follows from the classical maximum principle, shown next.

I.3.1. Theorem (Maximum Principle). Assume that $g$ is a smooth function with compact support on $\mathcal{S}$ which vanishes at $t=0$ and that $f^{0}$ is a smooth compactly supported function on $\mathcal{S}_{0}$. Let $f$ be a smooth solution to the initial value problem

$$
\begin{cases}L_{0} f=g & \text { in } S \\ f(\cdot, 0)=f^{0} & \text { on } S_{0}\end{cases}
$$


such that

$$
\|f\|_{C^{0}\left(\mathcal{S}_{T}\right)}<\infty
$$

for all $T>0$, with $\mathcal{S}_{T}=\mathcal{S}_{0} \times[0, T]$. Then, for any $T>0$ there exists a constant $C(T)$ depending only on $\nu$ and $T$ so that

$$
\|f\|_{C^{0}\left(\mathcal{S}_{T}\right)} \leq C(T)\left(\left\|f^{0}\right\|_{C^{0}\left(\mathcal{S}_{0}\right)}+\|g\|_{C^{0}(\mathcal{S})}\right) .
$$

Proof. Assume first that $g \equiv 0$. Fix a number $T>0$ and for $\mu \in(0,1)$ define the barrier function

$$
h_{\mu}(x, y, t)=M+\mu(t+1)\left[2 \nu(T+1)^{2}+2(T+1) x+y^{2}\right]
$$

with

$$
M=\left\|f^{0}\right\|_{C^{0}\left(\mathcal{S}_{0}\right)}
$$

It is easy to see that

$$
\left(h_{\mu}\right)_{t}-x\left(\left(h_{\mu}\right)_{x x}+\left(h_{\mu}\right)_{y y}\right)-\left(\nu h_{\mu}\right)_{x} \geq 0 \quad \text { on } \quad S_{T} .
$$

For $R>0$, we denote by $\mathcal{B}_{R}$ the box $\{0 \leq x \leq R,|y| \leq R\}$. We wish to show that when $R$ is sufficiently large, then

$$
f \leq h_{\mu} \quad \text { in } \quad \mathcal{B}_{R} \times[0, T]
$$

To see that $f \leq h_{\mu}$ at the lateral boundary of the cylinder $\mathcal{B}_{R} \times[0, T]$ is straightforward. Wherein, since the function $w=f-h_{\mu}$ satisfies

$$
w_{t}-x\left(w_{x x}+w_{y y}\right)-\nu w_{x} \leq 0 \quad \text { on } \quad \mathcal{B}_{R} \times[0, T]
$$

it follows from the classical maximum principle that $f \leq h_{\mu}$ in $\mathcal{B}_{R} \times[0, T]$. Notice that, because we have $x\left(w_{x x}+w_{y y}\right)=0$ at $x=0$ and the transport velocity $\nu$ is positive, the maximum of $w$ cannot occur at $x=0$. Therefore no boundary condition needs to be imposed at $x=0$. Now letting $R \rightarrow \infty$ and $\mu \rightarrow 0$, we conclude that $f(x, y, t) \leq M$ on $\mathcal{S}_{T}$. By similar arguments $f(x, y, t) \geq-M$ on $\mathcal{S}_{T}$ and hence the desired estimate holds true.

In the case that $g$ is not zero, the estimate can be proven by similar comparison arguments. If the function $g$ vanishes for $t \geq T_{0}$, the constant $C=C(T)$ can be taken to be independent of $T$.

I.4. Barrier functions. We need to construct barrier functions to estimate the derivatives of a solution to the homogeneous equation in a neighborhood of a point on the boundary. We imitate a familiar construction in the interior. If

$$
\varphi=\frac{1}{t}+\frac{1}{1-x^{2}-y^{2}},
$$

then for suitable constants $C<\infty$ and $c>0$ we have the barrier inequality

$$
\frac{\partial \varphi}{\partial t}>\varphi_{x x}+\varphi_{y y}-C \varphi^{2}+c
$$

as the reader can easily check. Note $\varphi \rightarrow \infty$ if $t \rightarrow 0$ or $x^{2}+y^{2} \rightarrow 1$. This barrier has been used frequently to do interior estimates of diffusion-reaction equations for diffusion in the Euclidean metric. For diffusion in our cycloidal metric we need a similar barrier. 
I.4.1. Definition. We say $\varphi$ satisfies the cycloidal barrier inequality with transport velocity $\nu$ if there exist suitable constants $C<\infty$ and $c>0$ such that

$$
\varphi_{t}>x\left(\varphi_{x x}+\varphi_{y y}\right)+\nu \varphi_{x}-C x \varphi^{2}+c \varphi^{3 / 2}+c .
$$

I.4.2. Definition. The barrier set $\Phi_{\nu}^{\gamma}$ for a given $\gamma<1$ is the collection of all functions $\varphi>0$ which

(a) are defined and smooth in a relatively open subset $U$ of the box $B_{1}$ where $U$ contains the box $B_{\gamma}$, and

(b) satisfy the cycloidal barrier inequality with transport velocity $\nu$, for some suitable constants $C<\infty$ and $c>0$ which may depend on $\varphi$.

Our objective in this section is to prove the following result.

I.4.3. Theorem. There exists a function $\varphi$ in the barrier set $\Phi_{\nu}^{\gamma}$ for any $\nu>0$ and $\gamma<1$ which is proper, in the sense that the sublevel sets $\{\varphi \leq B\}$ are compact for each constant $B<\infty$.

We begin with the following observation.

I.4.4. Theorem. The barrier set $\Phi_{\nu}^{\gamma}$ is a cone, in the sense that

(a) if $\varphi_{1}$ and $\varphi_{2}$ belong to $\Phi_{\nu}^{\gamma}$, so does $\varphi_{1}+\varphi_{2}$, and

(b) if $\varphi$ belongs to $\Phi_{\nu}^{\gamma}$ and $\lambda>0$ is a constant, then $\lambda \varphi$ belongs to $\Phi_{\nu}^{\gamma}$ also.

Proof. If $\varphi_{1}$ is defined in $U_{1}$ and $\varphi_{2}$ in $U_{2}$, then $\varphi_{1}+\varphi_{2}$ is defined in $U_{1} \cap U_{2}$, which is still a relatively open neighborhood of $(0,0,1)$. The inequality follows by adjusting the constants $C$ and $c$ using

$$
\left(\varphi_{1}+\varphi_{2}\right)^{2} \geq \varphi_{1}^{2}+\varphi_{2}^{2} \quad \text { and } \quad\left(\varphi_{1}+\varphi_{2}\right)^{3 / 2} \leq \sqrt{2}\left(\varphi_{1}^{3 / 2}+\varphi_{2}^{3 / 2}\right) .
$$

Scaling $\varphi$ also scales $C$ and $c$.

This allows us to construct $\varphi$ as a sum of pieces which will restrict the domain of $\varphi$ respectively to $\{t>0\},\{|y|<1-b t\}$ with $b$ small, and $\{x<1\}$ by having $\varphi \rightarrow \infty$ as we approach the new boundary. This will make $\varphi$ proper.

Now we find explicit barriers; first at $t=0$.

I.4.5. Theorem. For any $\nu>0$ we can find $a>0$ so that the function

$$
\varphi=\frac{1}{t(x+a t)}
$$

is in the barrier cone $\Phi_{\nu}^{\gamma}$.

Proof. We need to choose $C<\infty$ and $c>0$ so that

$$
C x \varphi^{2}-\nu \varphi_{x}>x \varphi_{x x}+c \varphi^{3 / 2}-\varphi_{t}+c
$$

for our given $\nu>0$, where $\varphi_{y y}=0$. Differentiating, we find we need

$$
\frac{C x}{t^{2}(x+a t)^{2}}+\frac{\nu}{t(x+a t)^{2}}>\frac{2 x}{t(x+a t)^{3}}+\frac{c}{t^{3 / 2}(x+a t)^{3 / 2}}+\frac{x+2 a t}{t^{2}(x+a t)^{2}}+c .
$$

Multiplying by $t^{2}(x+a t)^{2}$, we find that we need

$$
C x+\nu t>\frac{2 x t}{x+a t}+c t^{1 / 2}(x+a t)^{1 / 2}+x+2 a t+c t^{2}(x+a t)^{2} .
$$

Now we use $0<t \leq 1$ and $0 \leq x<1$ and

$$
\frac{2 x t}{x+a t} \leq \frac{2}{a} x
$$


and

$$
c t^{1 / 2}(x+a t)^{1 / 2} \leq c[x+(a+1) t]
$$

and

$$
c t^{2}(x+a t)^{2} \leq 4 c t
$$

if $a \leq 1$ to find that we only need $a \leq 1$ and

$$
C x+\nu t>\left(1+c+\frac{2}{a}\right) x+[2 a+c(5+a)] t
$$

which happens for all $x>0$ and $t>0$ if $a \leq 1$ and

$$
C>1+c+\frac{2}{a} \quad \text { and } \quad \nu>2 a+c(5+a) .
$$

Given $\nu>0$, choose $a>0$ and $c>0$ small enough to satisfy the second inequality, and then choose $C$ to satisfy the first. This proves $\varphi \in \Phi_{\nu}^{\gamma}$ for small $a>0$.

I.4.6. Theorem. For any $b>0$, the function

$$
\varphi=\frac{1}{(y-b t)^{2}}
$$

satisfies the barrier inequality on $\{0<y-b t<2\}$.

Proof. We need to find $C<\infty$ and $c>0$ with

$$
\varphi_{t}>x\left(\varphi_{x x}+\varphi_{y y}\right)+\nu \varphi_{x}-C x \varphi^{2}+c \varphi^{3 / 2}+c .
$$

Since $\varphi_{x}=0$ and $\varphi_{x x}=0$, it suffices to make

$$
\varphi_{t}>c \varphi^{3 / 2}+c \quad \text { and } \quad C \varphi^{2}>\varphi_{y y} .
$$

Differentiating, we find we need

$$
\frac{2 b}{(y-b t)^{3}}>\frac{c}{(y-b t)^{3}}+c
$$

which happens if $0<y-b t<2$ and $c<2 b / 9$, and we need

$$
\frac{C}{(y-b t)^{4}}>\frac{6}{(y-b t)^{4}}
$$

which happens if $C>6$.

I.4.7. Corollary. For any $b>0$ the function

$$
\varphi=\frac{1}{(1+y-b t)^{2}}+\frac{1}{(1-y-b t)^{2}}
$$

on $\{|y|<1-b t\}$ also belongs to the barrier cone $\Phi_{\nu}^{\gamma}$ provided $\nu>0$ and $b<1-\gamma$.

Proof. Since the inequality defining the barrier cone is preserved under the translation $y \rightarrow 1+y$ and the flip $1+y \rightarrow 1-y$, the result follows. Note that in the set $\{|y|<1-b t\}$ we have

$$
0<1+y-b t<2 \quad \text { and } \quad 0<1-y-b t<2
$$

as required by the previous Theorem. Also note that the set $\{|y|<1-b t, 0 \leq t \leq 1\}$ contains $\{|y|<\gamma\}$ and hence $B_{\gamma}$ provided $b<1-\gamma$.

Finally we construct a barrier function at $x \rightarrow 1$. 
I.4.8. Theorem. For any $\nu>0$ and $\gamma<1$ the function

$$
\varphi=\frac{1+t}{\left(1-x^{2}\right)^{2}}
$$

lies in the barrier cone $\Phi_{\nu}^{\gamma}$.

Proof. Since $\varphi_{y y}=0$, we need to find constants $C<\infty$ and $c>0$ with

$$
\varphi_{t}+C x \varphi^{2}>x \varphi_{x x}+\nu \varphi_{x}+c \varphi^{3 / 2}+c .
$$

Now when $0 \leq x<1$ and $0 \leq t \leq 1$ we have

$$
\varphi_{t}=\frac{1}{\left(1-x^{2}\right)^{2}}
$$

and

$$
x \varphi^{2} \geq \frac{x}{\left(1-x^{2}\right)^{4}}
$$

and

$$
\varphi^{3 / 2} \leq \frac{4}{\left(1-x^{2}\right)^{3}}
$$

and

$$
\varphi_{x}=\frac{4 x(1+t)}{\left(1-x^{2}\right)^{3}} \leq \frac{8 x}{\left(1-x^{2}\right)^{3}}
$$

and

$$
\varphi_{x x}=\frac{\left(4+20 x^{2}\right)(1+t)}{\left(1-x^{2}\right)^{4}} \leq \frac{48}{\left(1-x^{2}\right)^{4}} .
$$

Hence we only need

$$
\frac{1}{\left(1-x^{2}\right)^{2}}+\frac{C x}{\left(1-x^{2}\right)^{4}}>\frac{48 x}{\left(1-x^{2}\right)^{4}}+\frac{8 \nu x}{\left(1-x^{2}\right)^{3}}+\frac{4 c}{\left(1-x^{2}\right)^{3}}+c .
$$

Multiplying by $\left(1-x^{2}\right)^{4}$, we need

$$
\left(1-x^{2}\right)^{2}+C x>48 x+8 \nu x\left(1-x^{2}\right)+4 c\left(1-x^{2}\right)+c\left(1-x^{2}\right)^{4} .
$$

Since $0 \leq x<1$, we have

$$
8 \nu x\left(1-x^{2}\right) \leq 8 \nu x
$$

so it suffices to take $C \geq 49+8 \nu$ so that

$$
C x \geq\left[48 x+8 \nu x\left(1-x^{2}\right)\right]+x
$$

and to take $c$ so small that

$$
\left(1-x^{2}\right)^{2}+x>4 c\left(1-x^{2}\right)+c\left(1-x^{2}\right)^{4}
$$

which must happen on $0 \leq x \leq 1$ for some $c>0$ since the interval is compact and

$$
\left(1-x^{2}\right)^{2}+x>0
$$

on the entire interval. In fact if $x \leq 1 / 2$

$$
\left(1-x^{2}\right)^{2} \geq 9 / 16 \geq 1 / 2
$$

so

$$
\left(1-x^{2}\right)^{2}+x \geq 1 / 2
$$

on $0 \leq x \leq 1$, while $1-x^{2} \leq 1$, so it suffices to take $c \leq 1 / 10$. 
At last we can construct our proper barrier function

$$
\varphi=\frac{1}{t(x+a t)}+\frac{1}{(1+y-b t)^{2}}+\frac{1}{(1-y-b t)^{2}}+\frac{1+t}{\left(1-x^{2}\right)^{2}}
$$

for suitably small $a$ and $b$ as demonstrated. It is clear that $\varphi$ is proper since $\varphi \rightarrow \infty$ if $t \rightarrow 0$ or $x \rightarrow 1$ or $|y| \rightarrow 1-b t$. Since $b$ can be as small as we like, the domain of $\varphi$ still includes the box $B_{\gamma}$. This proves our Theorem I.4.3.

I.5. Local derivative estimates. Now we prove estimates on the derivatives $f_{x}$ and $f_{y}$ of a smooth solution $f$ of the equation for cycloidal diffusion with transport velocity $\nu>0$

$$
f_{t}=x\left(f_{x x}+f_{y y}\right)+\nu f_{x}+g
$$

for a forcing term $g$. We assume $f$ is defined in the box $B_{1}$ and that in the box we have bounds on $f, g, g_{x}$ and $g_{y}$, but this time we also need to assume a bound on $g_{y y}$. To see why, consider the evolution of the derivatives $f_{x}$ and $f_{y}$, which is given by

$$
f_{x t}=x\left(f_{x x x}+f_{x y y}\right)+(\nu+1) f_{x x}+f_{y y}+g_{y}
$$

and

$$
f_{y t}=x\left(f_{y x x}+f_{y y y}\right)+\nu f_{y x}+g_{y} .
$$

Note that $f_{y}$ satisfies the equation for cycloidal diffusion with transport velocity $\nu$ and forcing term $g_{y}$; while $f_{x}$ satisfies the equation for cycloidal diffusion with the new transport velocity $\nu+1$ and the forcing term $f_{y y}+g_{y}$. We will estimate $f_{y}$ from $g_{y}$, and then applying this again we will estimate $f_{y y}$ from $g_{y y}$, and use the estimate on $f_{y y}$ as well as that on $g_{x}$ to estimate $f_{x}$.

Consider first the function

$$
Y=\left(A+f^{2}\right) f_{y}^{2}
$$

for a large constant $A \geq 7$ we can choose later. The evolution of $Y$ is

$$
Y_{t}=2\left(A+f^{2}\right) f_{y} f_{y t}+2 f f_{y}^{2} f_{t}
$$

which becomes

$$
\begin{aligned}
Y_{t}=2\left(A+f^{2}\right) f_{y}\left[x\left(f_{y x x}+f_{y y y}\right)+\nu f_{y x}+g_{y}\right] \\
+2 f f_{y}^{2}\left[x\left(f_{x x}+f_{y y}\right)+\nu f_{x}+g\right] .
\end{aligned}
$$

It is easy to compute that if $A \geq 7,|f| \leq 1$ and

$$
Y=\left(A+f^{2}\right) f_{y}^{2}
$$

then

$$
Y_{x x}+Y_{y y} \geq 2\left(A+f^{2}\right) f_{y}\left(f_{y x x}+f_{y y y}\right)+2 f f_{y}^{2}\left(f_{x x}+f_{y y}\right)+f_{y}^{4} .
$$

Also

$$
Y_{x}=2\left(A+f^{2}\right) f_{y} f_{y x}+2 f f_{y}^{2} f_{x} .
$$

This gives us the estimate

$$
Y_{t} \leq x\left(Y_{x x}+Y_{y y}\right)+\nu Y_{x}-x f_{y}^{4}+2\left(A+f^{2}\right) f_{y} g_{y}+2 f f_{y}^{2} g .
$$

Then as before we use $|g| \leq 1$ and $\left|g_{y}\right| \leq 1$ and $A+f^{2} \leq 2 A$ to write

$$
Y_{t} \leq x\left(Y_{x x}+Y_{y y}\right)+\nu Y_{x}-x f_{y}^{4}+4 A\left|f_{y}\right|+2 f_{y}^{2}
$$


which gives

$$
Y_{t} \leq x\left(Y_{x x}+Y_{y y}\right)+\nu Y_{x}-x f_{y}^{4}+\left|f_{y}\right|^{3}+K A^{3 / 2}
$$

for some constant $K$ independent of $A$. Now

$$
\frac{Y}{2 A} \leq f_{y}^{2}=\frac{Y}{A+f^{2}} \leq \frac{Y}{A}
$$

so

$$
Y_{t} \leq x\left(Y_{x x}+Y_{y y}\right)+(\nu+1) Y_{x}-x\left(\frac{Y}{2 A}\right)^{2}+\left(\frac{Y}{A}\right)^{3 / 2}+K A^{3 / 2} .
$$

Let $\widetilde{Y}=Y / B$. Then

$$
\tilde{Y}_{t} \leq x\left(\tilde{Y}_{x x}+\tilde{Y}_{y y}\right)+\nu \widetilde{Y}_{x}-\frac{B}{4 A^{2}} x \widetilde{Y}^{2}+\frac{B^{1 / 2}}{A^{3 / 2}} \widetilde{Y}^{3 / 2}+\frac{K A^{3 / 2}}{B} .
$$

We want to make $B / A^{2}$ large, and both $B^{1 / 2} / A^{3 / 2}$ and $A^{3 / 2} / B$ small. Take $B=$ $A^{5 / 2}$. Then

$$
\widetilde{Y}_{t} \leq x\left(\widetilde{Y}_{x x}+\widetilde{Y}_{y y}\right)+\nu \widetilde{Y}_{x}-\frac{A^{1 / 2}}{4} x \widetilde{Y}^{2}+\frac{1}{A^{1 / 4}} \widetilde{Y}^{3 / 2}+\frac{K}{A} .
$$

Given any $C<\infty$ and $c>0$, we can take $A$ so large that

$$
\frac{A^{1 / 2}}{4} \geq C \quad \text { and } \quad \frac{1}{A^{1 / 4}} \leq c \quad \text { and } \quad \frac{K}{A} \leq c
$$

and get

$$
\widetilde{Y}_{t} \leq x\left(\widetilde{Y}_{x x}+\widetilde{Y}_{y y}\right)+\nu \widetilde{Y}_{x}-C x \widetilde{Y}^{2}+c \widetilde{Y}^{3 / 2}+c .
$$

Now our proper barrier function $\varphi$ gives a bound on $\widetilde{Y}$, which in turn gives a bound on $f_{y}$ in the smaller box $B_{\gamma}$ for any $\gamma<1$. The bound $\widetilde{Y} \leq \varphi$ follows from the maximum principle. Notice that in applying the maximum principle at the boundary we need to have $\nu \geq 0$, because if $\widetilde{Y} \leq \varphi$ in the interior and $\widetilde{Y}=\varphi$ at a boundary point, then $\widetilde{Y}_{x} \leq \varphi_{x}$ at the boundary point, and we need this inequality to enter with the correct sign. However if we try to prove the theorem for $\nu=0$, we have trouble with the barrier function inequality for $\varphi$ at the boundary. This proves the following.

I.5.1. Theorem. If $f$ is smooth and satisfies the cycloidal diffusion equation with transport velocity $\nu>0$

$$
f_{t}=x\left(f_{x x}+f_{y y}\right)+\nu f_{x}+g
$$

with forcing term $g$, and if

$$
|f| \leq 1 \quad \text { and } \quad|g| \leq 1 \quad \text { and } \quad\left|g_{y}\right| \leq 1
$$

on the box $\mathcal{B}_{1}=\{0 \leq x \leq 1,-1 \leq y \leq 1,0 \leq t \leq 1\}$, then for any $\gamma<1$

$$
\left|f_{y}\right| \leq C
$$

on the box

$$
\mathcal{B}_{\gamma}=\{0 \leq x \leq \gamma,-\gamma \leq y \leq \gamma, 1-\gamma \leq t \leq 1\}
$$

for some constant $C$ depending on $\nu>0$ and $\gamma<1$ but independent of $f$ and $g$. 
By dilation we get the following:

I.5.2. Corollary. If $f$ is smooth and satisfies the cycloidal diffusion equation with transport velocity $\nu>0$

$$
f_{t}=x\left(f_{x x}+f_{y y}\right)+\nu f_{x}+g
$$

with forcing term $g$, and if

$$
|f| \leq B \quad \text { and } \quad|g| \leq B \quad \text { and } \quad\left|g_{y}\right| \leq B / r
$$

on the box $\mathcal{B}_{r}$, then

$$
\left|f_{y}\right| \leq C B / r
$$

on the box $\mathcal{B}_{\gamma r}$ for any $\gamma<1$.

Since $f_{y}$ satisfies the cycloidal diffusion equation with transport velocity $\nu>0$

$$
f_{y t}=x\left(f_{y x x}+f_{y y y}\right)+\nu f_{y x}+g_{y}
$$

and forcing term $g_{y}$, we can apply this result again to get a bound on $f_{y y}$.

I.5.3. Corollary. If in addition

$$
\left|g_{y y}\right| \leq B / r^{2}
$$

on the box $\mathcal{B}_{r / 2}$, then

$$
\left|f_{y y}\right| \leq C B / r^{2}
$$

on the box $\mathcal{B}_{r / 4}$.

At last we are prepared to tackle the estimate for $f_{x}$. To simplify the discussion we will work on $\mathcal{B}_{1}$ and scale later. Suppose then that

$$
f_{t}=x\left(f_{x x}+f_{y y}\right)+\nu f_{x}+g
$$

on $\mathcal{B}_{1}$ and that we have bounds

$$
|f| \leq 1 \quad \text { and } \quad\left|f_{y y}\right| \leq 1 \quad \text { and } \quad|g| \leq 1 \quad \text { and } \quad\left|g_{x}\right| \leq 1
$$

on $\mathcal{B}_{1}$. Consider the quantity

$$
X=\left(A+f^{2}\right) f_{x}^{2}
$$

where $A$ is a large constant we can choose later. The evolution of $X$ is

$$
X_{t}=2\left(A+f^{2}\right) f_{x} f_{x t}+2 f f_{x}^{2} f_{t}
$$

and since

$$
f_{x t}=x\left(f_{x x x}+f_{x y y}\right)+(\nu+1) f_{x x}+f_{y y}+g_{x}
$$

we find

$$
\begin{aligned}
X_{t} & =2\left(A+f^{2}\right) f_{x}\left[x\left(f_{x x x}+f_{x y y}\right)+(\nu+1) f_{x x}+f_{y y}+g_{x}\right] \\
& +2 f f_{x}^{2}\left[x\left(f_{x x}+f_{y y}\right)+\nu f_{x}+g\right] .
\end{aligned}
$$

On the other hand, by direct computation if $A \geq 7$ and $|f| \leq 1$ we still have

$$
X_{x x}+X_{y y} \geq 2\left(A+f^{2}\right) f_{x}\left(f_{x x x}+f_{x y y}\right)+2 f f_{x}^{2}\left(f_{x x}+f_{y y}\right)+f_{x}^{4} .
$$

Also

$$
X_{x}=2\left(A+f^{2}\right) f_{x} f_{x x}+2 f f_{x}^{3} .
$$


Combining these gives

$$
\begin{aligned}
X_{t} \leq x\left(X_{x x}+X_{y y}\right) & +(\nu+1) X_{x}-x f_{x}^{4}-2 f f_{x}^{3} \\
& +2\left(A+f^{2}\right) f_{x}\left(f_{y y}+g_{x}\right)+2 f f_{x}^{2} g .
\end{aligned}
$$

When $A \geq 7$ and $|f| \leq 1$ we have $A+f^{2} \leq 2 A$, and we also assume $|g| \leq 1$ and $\left|g_{y}\right| \leq 1$ and $\left|f_{y y}\right| \leq 1$. This gives

$$
X_{t} \leq x\left(X_{x x}+X_{y y}\right)+(\nu+1) X_{x}-x f_{x}^{4}+2\left|f_{x}\right|^{3}+4 A\left|f_{x}\right|+2 f_{x}^{2}
$$

and so with some constant $K$ independent of $A$ we have

$$
X_{t} \leq x\left(X_{x x}+X_{y y}\right)+(\nu+1) X_{x}-x f_{x}^{4}+3\left|f_{x}\right|^{3}+K A^{3 / 2} .
$$

Now

so

$$
\frac{X}{2 A} \leq f_{x}^{2}=\frac{X}{A+f^{2}} \leq \frac{X}{A}
$$

$$
X_{t} \leq x\left(X_{x x}+X_{y y}\right)+(\nu+1) X_{x}-x\left(\frac{X}{2 A}\right)^{2}+3\left(\frac{X}{A}\right)^{3 / 2}+K A^{3 / 2} .
$$

Let $\widetilde{X}=X / B$. Then

$$
\widetilde{X}_{t} \leq x\left(\widetilde{X}_{x x}+\widetilde{X}_{y y}\right)+(\nu+1) \widetilde{X}_{x}-\frac{B}{4 A^{2}} x \widetilde{X}^{2}+\frac{3 B^{1 / 2}}{A^{3 / 2}} \widetilde{X}^{3 / 2}+\frac{K A^{3 / 2}}{B} .
$$

We want to make $B / A^{2}$ large, and both $B^{1 / 2} / A^{3 / 2}$ and $A^{3 / 2} / B$ small. Take $B=$ $A^{5 / 2}$. Then

$$
\widetilde{X}_{t} \leq x\left(\widetilde{X}_{x x}+\widetilde{X}_{y y}\right)+(\nu+1) \widetilde{X}_{x}-\frac{A^{1 / 2}}{4} x \widetilde{X}^{2}+\frac{3}{A^{1 / 4}} \widetilde{X}^{3 / 2}+\frac{K}{A} .
$$

Now given any $C<\infty$ and $c>0$, we can take $A$ so large that

$$
\frac{A^{1 / 2}}{4} \geq C \quad \text { and } \quad \frac{3}{A^{1 / 4}} \leq c \quad \text { and } \quad \frac{K}{A} \leq c
$$

and get

$$
\tilde{X}_{t} \leq x\left(\tilde{X}_{x x}+\tilde{X}_{y y}\right)+(\nu+1) \tilde{X}_{x}-C x \widetilde{X}^{2}+c \widetilde{X}^{3 / 2}+c .
$$

Now the maximum principle shows that $\widetilde{X} \leq \varphi$ for our barrier function $\varphi$. Since $\nu>0$ we also have $\nu+1>0$; therefore if the maximum occurs at the boundary, the term from $\widetilde{X}_{x} \leq \varphi_{x}$ comes in with the right sign. This establishes the following result.

I.5.4. Theorem. Suppose $f$ is smooth and satisfies the cycloidal diffusion equation with transport velocity $\nu>0$

$$
f_{t}=x\left(f_{x x}+f_{y y}\right)+\nu f_{x}+g
$$

with forcing term $g$ on the box

$$
\mathcal{B}_{1}=\{0 \leq x \leq 1,-1 \leq y \leq 1,0 \leq t \leq 1\} .
$$

If

$$
|f| \leq 1 \quad \text { and } \quad\left|f_{y y}\right| \leq 1 \quad \text { and } \quad|g| \leq 1 \quad \text { and } \quad\left|g_{x}\right| \leq 1
$$

on $\mathcal{B}_{1}$, then

$$
\left|f_{x}\right| \leq C
$$


on the box

$$
\mathcal{B}_{\gamma}=\{0 \leq x \leq \gamma,-\gamma \leq y \leq \gamma, 1-\gamma \leq t \leq 1\}
$$

with a constant $C$ which may depend on $\nu>0$ and $\gamma<1$ but is independent of $f$ and $g$.

By dilation we get the following.

I.5.5. Corollary. If $f$ and $g$ satisfy the equation on the box $\mathcal{B}_{r}$ and

$$
|f| \leq B \quad \text { and } \quad\left|f_{y y}\right| \leq B / r^{2} \quad \text { and } \quad|g| \leq B \quad \text { and } \quad\left|g_{x}\right| \leq B / r
$$

on $\mathcal{B}_{r}$, then

$$
\left|f_{x}\right| \leq C B / r
$$

on the box $\mathcal{B}_{\gamma r}$.

I.5.6. Corollary. If $f$ and $g$ satisfy the equation on the box $\mathcal{B}_{r}$ and if

$$
\begin{aligned}
& |f| \leq B \quad \text { and } \quad|g| \leq B \quad \text { and } \quad\left|g_{x}\right| \leq B / r \\
& \text { and } \quad\left|g_{y}\right| \leq B / r \quad \text { and } \quad\left|g_{y y}\right| \leq B / r^{2}
\end{aligned}
$$

on the box $\mathcal{B}_{r}$, then for any $\theta<1$

$$
\left|f_{x}\right| \leq C B / r \quad \text { and } \quad\left|f_{y}\right| \leq C B / r
$$

on the box $\mathcal{B}_{\theta r}$.

Proof. The bound on $f_{y}$ on $\mathcal{B}_{\gamma r}$ follows first from the equation for $f$ and the $y$ derivative estimate, which uses bounds on $g$ and $g_{y}$ on $\mathcal{B}_{r}$. The bound on $f_{y y}$ on $\mathcal{B}_{\gamma^{2} r}$ follows next from the equation for $f_{y}$ and the $y$-derivative estimate, which has $g_{y}$ as forcing term and hence uses bounds on $g_{y y}$ on $\mathcal{B}_{\gamma r}$ also. Now the bound on $f_{x}$ on $\mathcal{B}_{\gamma^{3} r}$ follows from the equation for $f$ and the $x$-derivative estimate, which uses the bound on $f_{y y}$ on $\mathcal{B}_{\gamma^{2} r}$ found above and the bound on $g_{x}$ on $\mathcal{B}_{\gamma^{2} r}$. Take $\gamma=\theta^{1 / 3}$ so $\theta=\gamma^{3}$. Continuing in this manner we can bound any derivative of $f$ we wish in any smaller box, using only bounds on $f$, and bounds on $g$ and the derivatives of $g$. If $f$ solves the homogeneous equation, we don't need to keep track.

I.5.7. Corollary. If $f$ is smooth and solves the homogeneous cycloidal diffusion equation with transport velocity $\nu>0$

$$
f_{t}=x\left(f_{x x}+f_{y y}\right)+\nu f_{x}
$$

then for any $i$ and $j$ and any $\gamma<1$ we can find a constant $C$ depending on $i$ and $j$ and $\nu>0$ and $\gamma<1$, but not on $f$ or on $r$, so that if $|f| \leq B$ on the box $\mathcal{B}_{r}$, then

$$
\left|D_{x}^{i} D_{y}^{j} f\right| \leq C B / r^{i+j}
$$

on the box $\mathcal{B}_{\gamma r}$.

Proof. Note that even if $f$ satisfies the homogeneous equation, then so does $f_{y}$ but $f_{x}$ does not. Thus even to prove the result for the homogeneous equation we need to consider the inhomogeneous case $g \neq 0$ and bound derivatives of $f$ in terms of derivatives of $g$. Since

$$
f_{x t}=x\left(f_{x x x}+f_{x y y}\right)+(\nu+1) f_{x x}+\left(f_{y y}+g_{x}\right)
$$

and

$$
f_{y t}=x\left(f_{y x x}+f_{y y y}\right)+\nu f_{y}+g_{y}
$$


we bound $D_{x}^{i} D_{y}^{j} f$ first by induction on the number $j$ of $y$-derivatives for fixed $i$, and then by induction on the number $i$ of $x$-derivatives.

I.6. Polynomial approximation. In order to make Schauder estimates we use a technique of approximation by a polynomial developed in [S1], [S2], [C], [W1] and [W2]. We will first prove a polynomial approximation theorem for the cycloidal diffusion operator with transport velocity $\nu>0$

$$
L_{0} f=f_{t}-x\left(f_{x x}+f_{y y}\right)-\nu f_{x}
$$

at points on the boundary. The differences will be that now time scales like space instead of like space squared, and we only take a polynomial of degree one in space and time instead of degree two in space, because at the boundary the diffusion goes to zero. We now let $\|f\|_{r}$ be the maximum of $f$ on the box $\mathcal{B}_{r}$.

I.6.1. Cycloidal Polynomial Approximation Theorem. There exists a constant $C$ with the following property. For every smooth function $f$ on the box $\mathcal{B}_{s}$ we can find a polynomial $p$ of degree one in space and time so that for every $r \leq s$

$$
\|f-p\|_{r} \leq C\left[\left(\frac{r}{s}\right)^{2}\|f\|_{s}+s\left\|L_{0} f\right\|_{s}\right] .
$$

Proof. By scaling it suffices to prove the result for $s=1$. Suppose then that $f$ is smooth on $\mathcal{B}_{1}$, and we shall show

$$
\|f-p\|_{r} \leq C\left[r^{2}\|f\|_{1}+\left\|L_{0} f\right\|_{1}\right] .
$$

Again choose a bump function $\psi$ on the set

$$
\mathcal{S}=\{0 \leq x<\infty,-\infty<y<\infty,-\infty<t \leq 1\}
$$

so that $\psi=0$ outside $\mathcal{B}_{1}$ and $\psi=1$ on $\mathcal{B}_{1 / 2}$. Let $L_{0} f=g$, and choose $h$ to be the unique bounded smooth solution on $\mathcal{S}$ of the equation

$$
L_{0} h=\psi g \text {. }
$$

Let $k=f-h$, and let $p$ be the Taylor polynomial of $k$

$$
p\left(\begin{array}{l}
x \\
y \\
t
\end{array}\right)=k\left(\begin{array}{l}
0 \\
0 \\
1
\end{array}\right)+k_{x}\left(\begin{array}{l}
0 \\
0 \\
1
\end{array}\right) x+k_{y}\left(\begin{array}{l}
0 \\
0 \\
1
\end{array}\right) y+k_{t}\left(\begin{array}{l}
0 \\
0 \\
1
\end{array}\right)(t-1)
$$

of degree 1 in space and time at the point $\left(\begin{array}{l}0 \\ 0 \\ 1\end{array}\right)$. For any point $\left(\begin{array}{l}x \\ y \\ t\end{array}\right)$ in $\mathcal{B}_{r}$ the remainder $k-p$ is given by terms involving derivatives of $k$ at another point in $\mathcal{B}_{r}$ of higher order times the corresponding monomial.

Now $k$ satisfies the homogeneous equation on $\mathcal{B}_{1 / 2}$, since

$$
L_{0} k=L_{0} f-L_{0} h=g-\psi g
$$

and $\psi=1$ on $\mathcal{B}_{1 / 2}$. Therefore we can bound any derivative of $k$ on $\mathcal{B}_{1 / 4}$ by a constant times the bound $\|k\|_{1}$ on $k$. The derivatives of $k$ at the point $\left(\begin{array}{l}0 \\ 0 \\ 1\end{array}\right)$ give a bound on the Taylor polynomial $p$,

$$
\|p\|_{1} \leq C\|k\|_{1} .
$$

To bound the remainder $k-p$ on $\mathcal{B}_{r}$, first suppose $r \leq 1 / 4$ so we have bounds on the derivatives of $k$ on $\mathcal{B}_{r}$. The terms in the remainder formula are monomials like $x^{2}$ and $x y$ and $x(t-1)$ and $(t-1)^{2}$ of higher degree than one in space and time. 
Since $0 \leq x \leq r$ and $|y| \leq r$ and $0 \leq 1-t \leq r$ on $\mathcal{B}_{r}$, all these monomials are bounded by $r^{2}$. This gives the remainder estimate

$$
\|k-p\|_{r} \leq C r^{2}\|k\|_{1}
$$

when $r \leq 1 / 4$. Of course if $r \geq 1 / 4$ it is much easier, $r^{2}$ doesn't matter and

$$
\|k-p\|_{r} \leq\|k-p\|_{1} \leq\|k\|_{1}+\|p\|_{1}
$$

and we already have $\|p\|_{1} \leq C\|k\|_{1}$.

We can estimate $\|h\|_{1}$ from the equation

$$
L_{0} h=h_{t}-x\left(h_{x x}+h_{y y}\right)-\nu h_{x}=\psi g
$$

using the maximum principle, since the maximum of $|\psi g|$ everywhere is no more than that of $g$ on $B_{1}$, namely $\|g\|_{1}$. Since $h=0$ for $t \leq 0$ because $\psi=0$ for $t \leq 0$, and the maximum of $h$ grows at most at a rate $\|g\|_{1}$ for $0 \leq t \leq 1$, we get

$$
\|h\|_{1} \leq\|g\|_{1} .
$$

Since $k=f-h$

$$
\|k\|_{1} \leq\|f\|_{1}+\|h\|_{1} \leq\|f\|_{1}+\|g\|_{1} .
$$

Now

$$
f-p=(f-k)+(k-p)=h+(k-p)
$$

so

$$
\|f-p\|_{r} \leq\|h\|_{r}+\|k-p\|_{r}
$$

and

$$
\|h\|_{r} \leq\|h\|_{1} \leq\|g\|_{1}
$$

and

$$
\|k-p\|_{r} \leq C r^{2}\|k\|_{1} \leq C r^{2}\left(\|f\|_{1}+\|g\|_{1}\right)
$$

which makes

$$
\|f-p\|_{r} \leq C\left(r^{2}\|f\|_{1}+\|g\|_{1}\right)
$$

which is the estimate we desire since $g=L_{0} f$.

I.7. Taylor remainder estimates. Now we derive Schauder estimates along the lines of Caffarelli, Safonov and Wang. Let

$$
L_{0} f=f_{t}-x\left(f_{x x}+f_{y y}\right)-\nu f_{x}
$$

be our operator this time, and let $\|f\|_{r}$ be the supremum of $f$ over the box $\mathcal{B}_{r}$.

I.7.1. Cycloidal Schauder Estimate. For each $\nu>0$ and each $\alpha$ in $0<\alpha<1$ there exists a constant $\mathcal{S}$ with the following property. If $f$ is a smooth function on the box $\mathcal{B}_{1}$ whose Taylor polynomial at $\left(\begin{array}{l}0 \\ 0 \\ 1\end{array}\right)$ of degree 1 in space and time is zero, then

$$
\sup _{0<r \leq 1} \frac{\|f\|_{r}}{r^{1+\alpha}} \leq \mathcal{S}\left(\|f\|_{1}+\sup _{0<r \leq 1} \frac{\left\|L_{0} f\right\|_{r}}{r^{\alpha}}\right)
$$


Proof. Since $f$ is smooth and its Taylor polynomial of degree 1 vanishes,

$$
\lim _{r \rightarrow 0} \frac{\|f\|_{r}}{r^{1+\alpha}}=0 ;
$$

likewise since the constant value of $L_{0} f$ at $\left(\begin{array}{l}0 \\ 0 \\ 1\end{array}\right)$ is zero,

$$
\lim _{r \rightarrow 0} \frac{\left\|L_{0} f\right\|_{r}}{r^{\alpha}}=0
$$

also. Thus both suprema are attained, although probably not at the same $r$. Suppose now that we let $r$ be the exact value of $u$ where

$$
\sup _{0<u \leq 1} \frac{\|f\|_{u}}{u^{1+\alpha}}=\frac{\|f\|_{r}}{r^{1+\alpha}}
$$

is attained.

First note that if we know $r \geq c$ for some constant $c>0$, we can take $S=1 / c^{1+\alpha}$ since $\|f\|_{r} \leq\|f\|_{1}$. Thus there is no problem unless $r$ is very small. In this case we shall choose $q$ much smaller than $r$ and $s$ much larger, of course with $s \leq 1$ which is why we need $r$ small. We shall estimate $S$ by making $q / r$ and $r / s$ both small enough (by amounts to be determined later).

Let $S$ be the best constant that works in the estimate, so that if (for ease)

$$
Q=\|f\|_{1}+\sup _{0<u \leq 1} \frac{\left\|L_{0} f\right\|_{u}}{u^{\alpha}}
$$

then

$$
\frac{\|f\|_{r}}{r^{1+\alpha}}=S Q
$$

We shall estimate $S$ from this.

By the cycloidal polynomial approximation theorem choose a polynomial $p$ of degree 1 in space and time so that for all $q \leq s$ we have

$$
\|f-p\|_{q} \leq C\left[\left(\frac{q}{s}\right)^{2}\|f\|_{s}+s\left\|L_{0} f\right\|_{s}\right]
$$

and since in particular $r \leq s$ we also have

$$
\|f-p\|_{r} \leq C\left[\left(\frac{r}{s}\right)^{2}\|f\|_{s}+s\left\|L_{0} f\right\|_{s}\right] .
$$

Since $p$ is a polynomial of degree 1

$$
\|p\|_{r} \leq C\left(\frac{r}{q}\right)\|p\|_{q}
$$

because we can estimate the coefficients of $p$ by $\|p\|_{q} / q$, and we can estimate $\|p\|_{r}$ by the coefficients times $r$. We also use

$$
\|f\|_{r} \leq\|f-p\|_{r}+\|p\|_{r}
$$

and

$$
\|p\|_{q} \leq\|f-p\|_{q}+\|f\|_{q}
$$

to get

$$
\|f\|_{r} \leq\|f-p\|_{r}+C\left(\frac{r}{q}\right)\left[\|f-p\|_{q}+\|f\|_{q}\right] .
$$


Then our approximation estimates on $f-p$ give

$$
\|f\|_{r} \leq C\left[\frac{r}{q}\|f\|_{q}+\left(\frac{r}{s}\right)^{2}\|f\|_{s}+\frac{r s}{q}\left\|L_{0} f\right\|_{s}\right] .
$$

From the definition of $S$

$$
\|f\|_{r}=S r^{1+\alpha} Q
$$

while

$$
\|f\|_{q} \leq S q^{1+\alpha} Q
$$

and

$$
\|f\|_{s} \leq S s^{1+\alpha} Q
$$

and from the definition of $Q$

$$
\left\|L_{0} f\right\|_{s} \leq s^{\alpha} Q
$$

Plug all this in and divide by $r^{1+\alpha} Q$ to get

$$
S \leq C\left[\left(\frac{q}{r}\right)^{\alpha}+\left(\frac{r}{s}\right)^{1-\alpha}\right] S+C \frac{s^{1+\alpha}}{q r^{\alpha}} .
$$

If $q / r$ and $r / s$ are small enough compared to the constant $C$, we get

$$
C\left[\left(\frac{q}{r}\right)^{\alpha}+\left(\frac{r}{s}\right)^{1-\alpha}\right] \leq \frac{1}{2}
$$

and this gives a bound on $S$ as desired.

Next we show how to remove the restriction on the Taylor polynomial vanishing, so that we get an estimate for a general function on the Taylor remainder. For a smooth function $f$ on the box $\mathcal{B}_{1}$ we let $T_{1} f$ denote the Taylor polynomial of $f$ of degree 1 in both space and time at the point $\left(\begin{array}{l}0 \\ 0 \\ 1\end{array}\right)$,

$$
T_{1} f\left(\begin{array}{l}
x \\
y \\
t
\end{array}\right)=f\left(\begin{array}{l}
0 \\
0 \\
1
\end{array}\right)+f_{x}\left(\begin{array}{l}
0 \\
0 \\
1
\end{array}\right) x+f_{y}\left(\begin{array}{l}
0 \\
0 \\
1
\end{array}\right) y+f_{t}\left(\begin{array}{l}
0 \\
0 \\
1
\end{array}\right)(t-1)
$$

and we let

$$
R_{1} f=f-T_{1} f
$$

denote the remainder. Likewise for a smooth function $g$ on $\mathcal{B}_{1}$ we let $T_{0} g$ denote the Taylor polynomial of degree 0 in both space and time at the point $\left(\begin{array}{l}0 \\ 0 \\ 1\end{array}\right)$,

$$
T_{0} g\left(\begin{array}{l}
x \\
y \\
t
\end{array}\right)=g\left(\begin{array}{l}
0 \\
0 \\
1
\end{array}\right)
$$

(so that $T_{0} g$ is a constant function), and we let

$$
R_{0} g=g-T_{0} g
$$

denote the remainder.

I.7.2. Corollary. For any smooth function $f$ on the box $B_{1}$

$$
\sup _{0<r \leq 1} \frac{\left\|R_{1} f\right\|_{r}}{r^{1+\alpha}} \leq S\left(\left\|R_{1} f\right\|_{1}+\sup _{0<r \leq 1} \frac{\left\|R_{0} L_{0} f\right\|_{r}}{r^{\alpha}}\right) .
$$


Proof. Apply Theorem I.7.1 to the remainder function $R_{1} f$, whose Taylor polynomial $T_{1} R_{1} f=0$.

Recall that now for cycloidal diffusion

$$
\|f\|_{r}=\|f\|_{C^{0}\left(\mathcal{B}_{r}\right)}=\sup _{P \in \mathcal{B}_{r}}|f(P)|
$$

and

$$
\|f\|_{C_{s}^{\beta}\left(\mathcal{B}_{r}\right)}=\|f\|_{C^{0}\left(\mathcal{B}_{r}\right)}+\sup _{P_{1}, P_{2} \in \mathcal{B}_{r}} \frac{\left|f\left(P_{1}\right)-f\left(P_{2}\right)\right|}{s\left[P_{1}, P_{2}\right]^{\beta}}
$$

with

$$
s\left[\left(\begin{array}{l}
x_{1} \\
y_{1} \\
t_{1}
\end{array}\right),\left(\begin{array}{l}
x_{2} \\
y_{2} \\
t_{2}
\end{array}\right)\right]=s\left[\left(\begin{array}{l}
x_{1} \\
y_{1}
\end{array}\right),\left(\begin{array}{l}
x_{2} \\
y_{2}
\end{array}\right)\right]+\sqrt{\left|t_{1}-t_{2}\right|}
$$

and

$$
c \frac{\left|x_{1}-x_{2}\right|+\left|y_{1}-y_{2}\right|}{\sqrt{x_{1}}+\sqrt{x_{2}}+\sqrt{\left|y_{1}-y_{2}\right|}} \leq s\left[\left(\begin{array}{l}
x_{1} \\
y_{1}
\end{array}\right),\left(\begin{array}{l}
x_{2} \\
y_{2}
\end{array}\right)\right] \leq C \frac{\left|x_{1}-x_{2}\right|+\left|y_{1}-y_{2}\right|}{\sqrt{x_{1}}+\sqrt{x_{2}}+\sqrt{\left|y_{1}-y_{2}\right|}}
$$

for constants $c>0$ and $C<\infty$. When one of the points is $P=\left(\begin{array}{l}0 \\ 0 \\ 1\end{array}\right)$, we have the simpler comparison

$$
c(\sqrt{|x|}+\sqrt{|y|}+\sqrt{|t-1|}) \leq s\left[\left(\begin{array}{l}
x \\
y \\
t
\end{array}\right),\left(\begin{array}{l}
0 \\
0 \\
1
\end{array}\right)\right] \leq C(\sqrt{|x|}+\sqrt{|y|}+\sqrt{|t-1|}),
$$

so for points $\left(\begin{array}{l}x \\ y \\ t\end{array}\right)$ in $\mathcal{B}_{r}$

$$
s\left[\left(\begin{array}{l}
x \\
y \\
t
\end{array}\right),\left(\begin{array}{l}
0 \\
0 \\
1
\end{array}\right)\right] \leq C \sqrt{r}
$$

for constants $c>0$ and $C<\infty$. Since $(\sqrt{r})^{2 \alpha}=r^{\alpha}$, our estimates in terms of $r^{\alpha}$ produce Hölder estimates of exponent $\beta=2 \alpha$. For example, for all smooth $g$ on $\mathcal{B}_{1}$

$$
\sup _{0<r \leq 1} \frac{\left\|R_{0} g\right\|_{r}}{r^{\alpha}} \leq C\|g\|_{C_{s}^{\beta}\left(\mathcal{B}_{1}\right)} .
$$

Now we can bound the Taylor polynomial $T_{1} f$, and hence the derivatives of $f$ at the point $\left(\begin{array}{l}0 \\ 0 \\ 1\end{array}\right)$ of degree 1 in both space and time.

I.7.3. Theorem. For every smooth function $f$ on the box $\mathcal{B}_{1}$ and every $\beta$ in $0<$ $\beta<1$

$$
\left\|T_{1} f\right\|_{C^{0}\left(\mathcal{B}_{1}\right)} \leq C\left(\|f\|_{C^{0}\left(\mathcal{B}_{1}\right)}+\left\|L_{0} f\right\|_{C_{s}^{\beta}\left(\mathcal{B}_{1}\right)}\right) .
$$

Proof. From Corollary I.7.2, for all $r$ in $0<r \leq 1$ and with $\alpha=\beta / 2$

$$
\left\|R_{1} f\right\|_{r} \leq S r^{1+\alpha}\left(\left\|R_{1} f\right\|_{1}+\left\|L_{0} f\right\|_{C_{s}^{\beta}\left(\mathcal{B}_{1}\right)}\right) .
$$

Since $T_{1} f$ is a polynomial of degree 1 in both space and time

$$
\left\|T_{1} f\right\|_{1} \leq \frac{C}{r}\left\|T_{1} f\right\|_{r}
$$


for all $r>0$. Then

$$
\left\|T_{1} f\right\|_{r} \leq\|f\|_{r}+\left\|R_{1} f\right\|_{r}
$$

and $\|f\|_{r} \leq\|f\|_{1}$, and

$$
\left\|R_{1} f\right\|_{1} \leq\|f\|_{1}+\left\|T_{1} f\right\|_{1} .
$$

Combining these gives

$$
\left\|T_{1} f\right\|_{1} \leq C r^{\alpha}\left\|T_{1} f\right\|_{1}+\frac{C}{r}\|f\|_{1}+C r^{\alpha}\left\|L_{0} f\right\|_{C_{s}^{\beta}\left(\mathcal{B}_{1}\right)} .
$$

Choose $r$ so small that $C r^{\alpha}<1 / 2$, and the bound on $\left\|T_{1} f\right\|_{C^{0}\left(\mathcal{B}_{1}\right)}=\left\|T_{1} f\right\|_{1}$ follows.

I.7.4. Corollary. If $i+j+k \leq 1$, then

$$
\left|D_{x}^{i} D_{y}^{j} D_{t}^{k} f\left(\begin{array}{l}
0 \\
0 \\
1
\end{array}\right)\right| \leq C\left(\|f\|_{C^{0}\left(\mathcal{B}_{1}\right)}+\left\|L_{0} f\right\|_{C_{s}^{\beta}\left(\mathcal{B}_{1}\right)}\right) .
$$

I.7.5. Corollary. We also have

$$
\sup _{0<r \leq 1} \frac{\left\|R_{1} f\right\|_{r}}{r^{1+\alpha}} \leq C\left(\|f\|_{C^{0}\left(\mathcal{B}_{1}\right)}+\left\|L_{0} f\right\|_{C_{s}^{\beta}\left(\mathcal{B}_{1}\right)}\right)
$$

for all smooth $f$ on $B_{1}$, with $0<\beta=2 \alpha<1$.

Proof. We use the bound in Corollary I.7.4 and

$$
\left\|R_{1} f\right\|_{1} \leq\|f\|_{1}+\left\|T_{1} f\right\|_{1}
$$

and the bound in Theorem I.7.3 on $\left\|T_{1} f\right\|_{1}$.

Next we show that we can replace the norm $\left\|L_{0} f\right\|_{C_{s}^{\beta}\left(\mathcal{B}_{1}\right)}$ in Theorem I.7.3 and Corollary I.7.4 by the seminorm $\left\|L_{0} f\right\|_{H_{s}^{\beta}\left(\mathcal{B}_{1}\right)}$.

I.7.6. Theorem. For every smooth function $f$ on the box $\mathcal{B}_{1}$ and every $\alpha$ in $0<$ $\alpha<1$

$$
\left\|T_{1} f\right\|_{C^{0}\left(\mathcal{B}_{1}\right)} \leq C\left(\|f\|_{C^{0}\left(\mathcal{B}_{1}\right)}+\left\|L_{0} f\right\|_{H_{s}^{\beta}\left(\mathcal{B}_{1}\right)}\right) .
$$

Proof. From Corollary I.7.2 since

$$
\sup _{0<r \leq 1} \frac{\left\|R_{0} L_{0} f\right\|_{r}}{r^{\alpha}} \leq C\left\|L_{0} f\right\|_{H_{s}^{\beta}\left(\mathcal{B}_{1}\right)}
$$

we have

$$
\left\|R_{1} f\right\|_{C^{0}\left(\mathcal{B}_{r}\right)} \leq C r^{1+\alpha}\left(\left\|R_{1} f\right\|_{C^{0}\left(\mathcal{B}_{1}\right)}+\left\|L_{0} f\right\|_{H_{s}^{\beta}\left(B_{1}\right)}\right) .
$$

Since $T_{1} f$ is a polynomial of degree 1 in both space and time

$$
\left\|T_{1} f\right\|_{C^{0}\left(\mathcal{B}_{1}\right)} \leq \frac{C}{r}\left\|T_{1} f\right\|_{C^{0}\left(\mathcal{B}_{r}\right)}
$$

for all $r>0$. Then

$$
\left\|T_{1} f\right\|_{C^{0}\left(\mathcal{B}_{r}\right)} \leq\|f\|_{C^{0}\left(\mathcal{B}_{r}\right)}+\left\|R_{1} f\right\|_{C^{0}\left(\mathcal{B}_{r}\right)}
$$

and

$$
\|f\|_{C^{0}\left(\mathcal{B}_{r}\right)} \leq\|f\|_{C^{0}\left(\mathcal{B}_{1}\right)}
$$


and

$$
\left\|R_{1} f\right\|_{C^{0}\left(\mathcal{B}_{1}\right)} \leq\|f\|_{C^{0}\left(\mathcal{B}_{1}\right)}+\left\|T_{1} f\right\|_{C^{0}\left(\mathcal{B}_{1}\right)} .
$$

Combining these gives

$$
\left\|T_{1} f\right\|_{C^{0}\left(\mathcal{B}_{1}\right)} \leq C r^{\alpha}\left\|T_{1} f\right\|_{C^{0}\left(\mathcal{B}_{1}\right)}+\frac{C}{r}\|f\|_{C^{0}\left(\mathcal{B}_{1}\right)}+C r^{\alpha}\left\|L_{0} f\right\|_{H_{s}^{\beta}\left(\mathcal{B}_{1}\right)} .
$$

Choose $r$ so small that $C r^{\alpha}<1 / 2$, and the bound on $T_{1} f$ follows.

I.7.7. Corollary. If $i+j+k \leq 1$, then

$$
\left|D_{x}^{i} D_{y}^{j} D_{t}^{k} f\left(\begin{array}{l}
0 \\
0 \\
1
\end{array}\right)\right| \leq C\left(\|f\|_{C^{0}\left(\mathcal{B}_{1}\right)}+\left\|L_{0} f\right\|_{H_{s}^{\beta}\left(\mathcal{B}_{1}\right)}\right) .
$$

I.7.8. Corollary. We also have

$$
\left\|R_{1} f\right\|_{C^{0}\left(\mathcal{B}_{r}\right)} \leq C r^{1+\alpha}\left(\|f\|_{C^{0}\left(\mathcal{B}_{1}\right)}+\left\|L_{0} f\right\|_{H_{s}^{\beta}\left(\mathcal{B}_{1}\right)}\right)
$$

for all $r$ in $0 \leq r \leq 1$.

Proof. We use the bound in Corollary I.7.2 and

$$
\left\|R_{1} f\right\|_{C^{0}\left(\mathcal{B}_{1}\right)} \leq\|f\|_{C^{0}\left(\mathcal{B}_{1}\right)}+\left\|T_{1} f\right\|_{C^{0}\left(\mathcal{B}_{1}\right)}
$$

and the bound on $T_{1} f$ from Theorem I.7.6.

I.8. Schauder estimates in the interior. Given a point $P=\left(\begin{array}{l}x_{0} \\ y_{0} \\ t_{0}\end{array}\right)$ we define the parabolic cylinder $\mathcal{C}_{r}(P)$ of radius $r$ around $P$ to be the set

$$
\mathcal{C}_{r}(P)=\left\{\left(\begin{array}{l}
x \\
y \\
t
\end{array}\right): \begin{array}{c}
\left|x-x_{0}\right|^{2}+\left|y-y_{0}\right|^{2} \leq r^{2} \\
t_{0}-r^{2} \leq t \leq t_{0}
\end{array}\right\} .
$$

Then

$$
\mathcal{C}_{r}=\mathcal{C}_{r}\left(\begin{array}{l}
0 \\
0 \\
1
\end{array}\right)
$$

If $P \in \mathcal{C}_{1 / 2}$, then $\mathcal{C}_{1 / 2}(P) \subseteq \mathcal{C}_{1}$. If

$$
I=(i, j, k)
$$

is a multi-index, we let

$$
D^{I} f=D_{x}^{i} D_{y}^{j} D_{t}^{k} f .
$$

We review the classical Schauder estimates for the heat operator

$$
H f=f_{t}-\left(f_{x x}+f_{y y}\right) .
$$

I.8.1. Theorem. For any $r<1$ there exists a constant $C<\infty$ depending on $r$ with the following property. If $f$ is any smooth function on the cylinder $\mathcal{C}_{1}$, then

$$
\|f\|_{C^{2+\alpha}\left(\mathcal{C}_{r}\right)} \leq C\left(\|f\|_{C^{0}\left(\mathcal{C}_{1}\right)}+\|H f\|_{C^{\alpha}\left(\mathcal{C}_{1}\right)}\right) .
$$


We first prove interior Schauder estimates for the cycloidal diffusion operator with transport velocity $\nu$

$$
L_{0} f=f_{t}-x\left(f_{x x}+f_{y y}\right)-\nu f_{x}
$$

in a small cylinder around the interior point $Q=\{x=1, y=0, t=1\}$. Since

$$
H f=f_{t}-\left(f_{x x}+f_{y y}\right)
$$

we have

$$
H f-L_{0} f=(x-1)\left(f_{x x}+f_{y y}\right)+\nu f_{x} .
$$

On the cylinder $\mathcal{C}_{\lambda}(Q)$ with $\lambda<1$

$$
\left\|H f-L_{0} f\right\|_{C^{\alpha}\left(\mathcal{C}_{\lambda}(Q)\right)} \leq C \lambda\|f\|_{C^{2+\alpha}\left(\mathcal{C}_{\lambda}(Q)\right)}+C\|f\|_{C^{1+\alpha}\left(\mathcal{C}_{\lambda}(Q)\right)}
$$

where $C$ depends on $\nu$ but not $\lambda$. We have

$$
\|f\|_{C^{2+\alpha}\left(\mathcal{C}_{1 / 4}(Q)\right)} \leq C\left(\|f\|_{C^{0}\left(\mathcal{C}_{1}(Q)\right)}+\|H f\|_{C^{\alpha}\left(\mathcal{C}_{1}(Q)\right)}\right)
$$

by translating Theorem I.8.1, with a constant independent of $\lambda$. If $f$ has support in $\mathcal{C}_{\lambda}$, so $f$ is defined for all $x$ and $y$ and $t \leq 1$ and vanishes if $|x-1| \geq \lambda$ or $|y| \geq \lambda$ or $t \leq 1-\lambda^{2}$, then for all $\mu \geq \lambda$ any norm of $f$ on $\mathcal{C}_{\mu}$ is the same as its norm on $\mathcal{C}_{\lambda}$. Thus

$$
\|f\|_{C^{2+\alpha}\left(\mathcal{C}_{\lambda}(Q)\right)} \leq C\left(\|f\|_{C^{0}\left(\mathcal{C}_{\lambda}(Q)\right)}+\|H f\|_{C^{\alpha}\left(\mathcal{C}_{\lambda}(Q)\right)}\right)
$$

with a constant independent of $\lambda$. We can estimate

$$
\|H f\|_{C^{\alpha}\left(\mathcal{C}_{\lambda}(Q)\right)} \leq\left\|L_{0} f\right\|_{C^{\alpha}\left(\mathcal{C}_{\lambda}(Q)\right)}+\left\|H f-L_{0} f\right\|_{C^{\alpha}\left(\mathcal{C}_{\lambda}(Q)\right)} .
$$

This gives

$$
\|f\|_{C^{2+\alpha}\left(\mathcal{C}_{\lambda}(Q)\right)} \leq C \lambda\|f\|_{C^{2+\alpha}\left(\mathcal{C}_{\lambda}(Q)\right)}+C\|f\|_{C^{1+\alpha}\left(\mathcal{C}_{\lambda}(Q)\right)}+C\left\|L_{0} f\right\|_{C^{\alpha}\left(\mathcal{C}_{\lambda}(Q)\right)} .
$$

Choose $\lambda$ so small that $C \lambda \leq 1 / 2$; then we get the following result:

I.8.2. Theorem. There exists a number $\lambda>0$ and a constant $C$ with the following property. For every function $f$ with support in the cylinder $\mathcal{C}_{\lambda}(Q)$ with $Q=\left(\begin{array}{l}1 \\ 0 \\ 1\end{array}\right)$ we have

$$
\|f\|_{C^{2+\alpha}\left(\mathcal{C}_{\lambda}(Q)\right)} \leq C\left(\|f\|_{C^{1+\alpha}\left(\mathcal{C}_{\lambda}(Q)\right)}+\left\|L_{0} f\right\|_{C^{\alpha}\left(\mathcal{C}_{\lambda}(Q)\right)}\right) .
$$

We can improve the lower order norm.

I.8.3. Corollary. We also have

$$
\|f\|_{C^{2+\alpha}\left(\mathcal{C}_{\lambda}(Q)\right)} \leq C\left(\|f\|_{C^{0}\left(\mathcal{C}_{\lambda}(Q)\right)}+\left\|L_{0} f\right\|_{C^{\alpha}\left(\mathcal{C}_{\lambda}(Q)\right)}\right) .
$$

Proof. For any $\varepsilon>0$ we can find a constant $C_{\varepsilon}$ so that if $f$ has support in $\mathcal{C}_{\lambda}(Q)$,

$$
\|f\|_{C^{1+\alpha}\left(\mathcal{C}_{\lambda}(Q)\right)} \leq \varepsilon\|f\|_{C^{2+\alpha}\left(\mathcal{C}_{\lambda}(Q)\right)}+C_{\varepsilon}\|f\|_{C^{0}(\mathcal{C} \lambda(Q))}
$$

by interpolation theory. If $C \varepsilon \leq 1 / 2$, we can get the desired bound.

We also observe that it suffices to use the semi-norm $H^{\alpha}$ on $L_{0} f$.

I.8.4. Corollary. We also have

$$
\|f\|_{C^{2+\alpha}\left(\mathcal{C}_{\lambda}(Q)\right)} \leq C\left(\|f\|_{C^{0}\left(\mathcal{C}_{\lambda}(Q)\right)}+\left\|L_{0} f\right\|_{H^{\alpha}\left(\mathcal{C}_{\lambda}(Q)\right)}\right) .
$$

Proof. Since $g=L_{0} f$ also has support in $\mathcal{C}_{\lambda}(Q)$

$$
\|g\|_{C^{0}\left(\mathcal{C}_{\lambda}(Q)\right)} \leq C\|g\|_{H^{\alpha}\left(\mathcal{C}_{\lambda}(Q)\right)}
$$

comparing the point where $|g|$ is a maximum to a point where $g=0$. 
We can also drop the restriction that $f$ has support in $\mathcal{C}_{\lambda}(Q)$.

I.8.5. Theorem. There exists $\lambda>0$ such that for any $\mu<\lambda$ and any smooth function $f$ on $\mathcal{C}_{\lambda}(Q)$ without restriction on its support

$$
\|f\|_{C^{2+\alpha}\left(\mathcal{C}_{\mu}(Q)\right)} \leq C\left(\|f\|_{C^{0}\left(\mathcal{C}_{\lambda}(Q)\right)}+\left\|L_{0} f\right\|_{H^{\alpha}\left(\mathcal{C}_{\lambda}(Q)\right)}\right) .
$$

Proof. Choose $\nu, \rho, \sigma$ and $\theta$ with

$$
\mu<\nu<\rho<\sigma<\lambda<\theta
$$

and choose bump functions $\varphi$ and $\psi$ so that

$$
\left\{\begin{array}{lll}
\varphi=1 & \text { on } & \mathcal{C}_{\nu} \\
\varphi=0 & \text { off } & \mathcal{C}_{\rho}
\end{array}\right.
$$

and

$$
\left\{\begin{array}{lll}
\psi=1 & \text { on } & \mathcal{C}_{\sigma}, \\
\psi=0 & \text { off } & \mathcal{C}_{\lambda} .
\end{array}\right.
$$

Solve for the unique bounded function $h$ with

$$
L_{0} h=\varphi L_{0} f .
$$

By the maximum principle

$$
\|h\|_{C^{0}\left(\mathcal{C}_{\theta}(Q)\right)} \leq C\left\|\varphi L_{0} f\right\|_{C^{0}\left(C_{\rho}(Q)\right)} \leq C\left\|L_{0} f\right\|_{H^{\alpha}\left(\mathcal{C}_{\rho}(Q)\right)}
$$

because $\varphi L_{0} f$ has compact support in $\mathcal{C}_{\rho}(Q)$. Since $L_{0} h=0$ off of $\mathcal{C}_{\rho}$, the interior derivative estimates on solutions of the homogeneous equation give

$$
\begin{aligned}
\|h\|_{C^{1+\alpha}\left(\mathcal{C}_{\lambda}(Q)-\mathcal{C}_{\sigma}(Q)\right)} & \leq\|h\|_{C^{2}\left(\mathcal{C}_{\lambda}(Q)-\mathcal{C}_{\sigma}(Q)\right)} \\
& \leq\|h\|_{C^{0}\left(\mathcal{C}_{\theta}(Q)-\mathcal{C}_{\rho}(Q)\right)} \leq C\left\|L_{0} f\right\|_{H^{\alpha}\left(\mathcal{C}_{\rho}(Q)\right)}
\end{aligned}
$$

since we can cover $\mathcal{C}_{\lambda}(Q)-\mathcal{C}_{\sigma}(Q)$ with a finite number of balls in slightly larger balls in $\mathcal{C}_{\theta}(Q)-\mathcal{C}_{\rho}(Q)$. Since the commutator $\left[L_{0}, \psi\right]$ has support in $\mathcal{C}_{\lambda}(Q)-\mathcal{C}_{\sigma}(Q)$ and degree 1 in space and zero in time

$$
\left\|\left[L_{0}, \psi\right] h\right\|_{C^{\alpha}\left(\mathcal{C}_{\lambda}(Q)\right)} \leq C\|h\|_{C^{1+\alpha}\left(\mathcal{C}_{\lambda}(Q)-\mathcal{C}_{\sigma}(Q)\right)} \leq C\left\|L_{0} f\right\|_{H^{\alpha}\left(\mathcal{C}_{\rho}(Q)\right)} .
$$

Now

$$
L_{0} \psi h=\psi L_{0} h+\left[L_{0}, \psi\right] h
$$

and $\psi \varphi=\varphi$ so

$$
L_{0} \psi h=\varphi L_{0} f+\left[L_{0}, \psi\right] h .
$$

Since $\psi h$ has compact support in $\mathcal{C}_{\lambda}(Q)$

$$
\|\psi h\|_{C^{2+\alpha}\left(\mathcal{C}_{\lambda}(Q)\right)} \leq C\left(\|\psi h\|_{\mathcal{C}^{0}\left(\mathcal{C}_{\lambda}(Q)\right)}+\left\|L_{0} \psi h\right\|_{H^{\alpha}\left(\mathcal{C}_{\lambda}(Q)\right)}\right)
$$

by Corollary I.8.4. Therefore, since

$$
\left\|L_{0} \psi h\right\|_{H^{\alpha}\left(\mathcal{C}_{\lambda}(Q)\right)} \leq\left\|\varphi L_{0} f\right\|_{H^{\alpha}\left(\mathcal{C}_{\lambda}(Q)\right)}+\left\|\left[L_{0}, \psi\right] h\right\|_{H^{\alpha}\left(\mathcal{C}_{\lambda}(Q)\right)} \leq C\left\|L_{0} f\right\|_{H^{\alpha}\left(\mathcal{C}_{\rho}(Q)\right)}
$$

and

$$
\|\psi h\|_{C^{0}\left(\mathcal{C}_{\lambda}(Q)\right)} \leq\|h\|_{C^{0}\left(\mathcal{C}_{\lambda}(Q)\right)} \leq C\left\|L_{0} f\right\|_{H^{\alpha}\left(\mathcal{C}_{\rho}(Q)\right)},
$$

we get

$$
\|h\|_{C^{2+\alpha}\left(\mathcal{C}_{\mu}(Q)\right)} \leq\|\psi h\|_{C^{2+\alpha}\left(\mathcal{C}_{\lambda}(Q)\right)} \leq C\left\|L_{0} f\right\|_{H^{\alpha}\left(\mathcal{C}_{\rho}(Q)\right)} .
$$


Next let $k=f-h$; then

$$
L_{0} k=L_{0} f-L_{0} h=(1-\varphi) L_{0} f
$$

so $L k=0$ on $\mathcal{C}_{\nu}$. By the interior derivative estimates on solutions of the homogeneous equation

$$
\|k\|_{C^{2+\alpha}\left(\mathcal{C}_{\mu}(Q)\right)} \leq C\|k\|_{C^{3}\left(\mathcal{C}_{\mu}(Q)\right)} \leq C\|k\|_{C^{0}\left(\mathcal{C}_{\nu}(Q)\right)} \leq C\|k\|_{C^{0}\left(\mathcal{C}_{\lambda}(Q)\right)} .
$$

Now $k=f-h$ so

$$
\|k\|_{C^{0}\left(\mathcal{C}_{\lambda}(Q)\right)} \leq\|f\|_{C^{0}\left(\mathcal{C}_{\lambda}(Q)\right)}+\|h\|_{C^{0}\left(\mathcal{C}_{\lambda}(Q)\right)}
$$

and once again

$$
\|h\|_{C^{0}\left(\mathcal{C}_{\lambda}(Q)\right)} \leq C\left\|L_{0} f\right\|_{H^{\alpha}\left(\mathcal{C}_{\rho}(Q)\right)}
$$

so we get

$$
\|k\|_{C^{2+\alpha}\left(\mathcal{C}_{\mu}(Q)\right)} \leq C\left(\|f\|_{C^{0}\left(\mathcal{C}_{\lambda}(Q)\right)}+\left\|L_{0} f\right\|_{H^{\alpha}\left(\mathcal{C}_{\rho}(Q)\right)}\right) .
$$

Now $f=h+k$ so

$$
\begin{aligned}
\|f\|_{C^{2+\alpha}\left(\mathcal{C}_{\mu}(Q)\right)} & \leq\|h\|_{C^{2+\alpha}\left(\mathcal{C}_{\mu}(Q)\right)}+\|k\|_{C^{2+\alpha}\left(\mathcal{C}_{\mu}(Q)\right)} \\
& \leq C\left(\|f\|_{C^{0}\left(\mathcal{C}_{\lambda}(Q)\right)}+\left\|L_{0} f\right\|_{H^{\alpha}\left(\mathcal{C}_{\rho}(Q)\right)}\right)
\end{aligned}
$$

as desired.

On the cylinder $\mathcal{C}_{\lambda}(Q)$ the metric

$$
d s^{2}=\frac{d x^{2}+d y^{2}}{2 x}
$$

is equivalent to the Euclidean metric since $|x-1| \leq \lambda$ and $\lambda$ is small. This gives the following restatement, replacing $\alpha$ by $\beta$ and $H^{\alpha}$ by $H_{s}^{\beta}$ in Theorem I.8.5.

I.8.6. Corollary. We also have

$$
\|f\|_{C_{s}^{2+\beta}\left(\mathcal{C}_{\mu}(Q)\right)} \leq C\left(\|f\|_{C^{0}\left(\mathcal{C}_{\lambda}(Q)\right)}+\left\|L_{0} f\right\|_{H_{s}^{\beta}\left(\mathcal{C}_{\lambda}(Q)\right)}\right) .
$$

For dilation purposes we introduce the semi-norm $H_{s}^{2+\beta}$ on a set $\mathcal{A}$ :

$$
\begin{aligned}
\|f\|_{H_{s}^{2+\beta}(\mathcal{A})}=\left\|x f_{x x}\right\|_{H_{s}^{\beta}(\mathcal{A})} & +\left\|x f_{x y}\right\|_{H_{s}^{\beta}(\mathcal{A})}+\left\|x f_{y y}\right\|_{H_{s}^{\beta}(\mathcal{A})} \\
& +\left\|f_{x}\right\|_{H_{s}^{\beta}(\mathcal{A})}+\left\|f_{y}\right\|_{H_{s}^{\beta}(\mathcal{A})}+\left\|f_{t}\right\|_{H_{s}^{\beta}(\mathcal{A})} .
\end{aligned}
$$

Clearly $H_{s}^{2+\beta}$ is weaker than $C_{s}^{2+\beta}$, so

$$
\|f\|_{H_{s}^{2+\beta}\left(\mathcal{C}_{\mu}(Q)\right)} \leq C\left(\|f\|_{C^{0}\left(\mathcal{C}_{\lambda}(Q)\right)}+\left\|L_{0} f\right\|_{H_{s}^{\beta}\left(\mathcal{C}_{\mu}(Q)\right)}\right) .
$$

Each of these norms behaves well under dilation. If we dilate space and time by a constant factor $r$, then $L_{0} f$ dilates by $r$, the $C^{0}$ norm is unchanged, the $H_{s}^{\beta}$ norm dilates by $s^{\beta}=r^{\alpha}$ with $\alpha=\beta / 2$, and the $H_{s}^{2+\beta}$ norm dilates by $r s^{\beta}=r^{1+\alpha}$.

Let $Q_{r}$ be the point

$$
Q_{r}=\{x=r, y=0, t=1\}
$$

and let

$$
\mathcal{A}_{\lambda r}\left(Q_{r}\right)=\left\{\begin{array}{c}
(x-r)^{2}+y^{2} \leq \lambda^{2} r^{2} \\
1-\lambda^{2} r \leq t \leq 1
\end{array}\right\}
$$


be the cylinder obtained by scaling the cylinder

$$
\mathcal{C}_{\lambda}(Q)=\left\{\begin{array}{c}
(x-1)^{2}+y^{2} \leq \lambda^{2} \\
1-\lambda^{2} \leq t \leq 1
\end{array}\right\}
$$

by $x \rightarrow r x, y \rightarrow r y,(1-t) \rightarrow r(1-t)$.

I.8.7. Corollary. There exists a $\lambda>0$ such that for every $\mu<\lambda$ and every $\beta$ in $0<\beta<1$ we can find a constant $C$ with the following property. For every $r>0$ and every smooth function $f$ on the cylinder $\mathcal{A}_{\lambda r}\left(Q_{r}\right)$

$$
\|f\|_{H_{s}^{2+\beta}\left(\mathcal{A}_{\mu r}\left(Q_{r}\right)\right)} \leq C\left(\frac{1}{r^{1+\alpha}}\|f\|_{C^{0}\left(\mathcal{A}_{\lambda r}\left(Q_{r}\right)\right)}+\left\|L_{0} f\right\|_{H_{s}^{\beta}\left(\mathcal{A}_{\lambda r}\left(Q_{r}\right)\right)}\right)
$$

with $\alpha=\beta / 2$.

From this Schauder estimate we can work backwards to get a Taylor remainder estimate. Let $T_{2,1}^{Q_{r}} f$ denote the Taylor polynomial of $f$ of degree 2 in space and 1 in time at the point $Q_{r}$, and let

$$
R_{2,1}^{Q_{r}} f=f-T_{2,1}^{Q_{r}} f
$$

be the Taylor remainder at $Q_{r}$. By the remainder formula we can express $R_{2,1}^{Q_{r}} f$ in terms of the differences of derivatives $f_{x x}, f_{x y}, f_{y y}, f_{t}$ between $Q_{r}$ and the near by points, so that, as we see by dilating from $\mathcal{A}_{\mu}(Q)$,

$$
\left\|R_{2,1}^{Q_{r}} f\right\|_{C^{0}\left(\mathcal{A}_{\mu r}(Q)\right)} \leq C r^{1+\alpha}\|f\|_{H_{s}^{2+\beta}\left(\mathcal{A}_{\mu r}\left(Q_{r}\right)\right)} .
$$

Combining this with the previous estimate gives this corollary.

I.8.8. Corollary. We also have (with $\alpha=\beta / 2$ )

$$
\left\|R_{2,1}^{Q_{r}} f\right\|_{C^{0}\left(\mathcal{A}_{\mu r}\left(Q_{r}\right)\right)} \leq C\left(\|f\|_{C^{0}\left(\mathcal{A}_{\lambda r}\left(Q_{r}\right)\right)}+r^{1+\alpha}\left\|L_{0} f\right\|_{H_{s}^{\beta}\left(\mathcal{A}_{\lambda r}\left(Q_{r}\right)\right)}\right) .
$$

I.9. Schauder estimates near the boundary. We can obtain Schauder estimates near the boundary comparing the second derivatives $f_{x x}, f_{x y}, f_{y y}$ at a point $P=\left(\begin{array}{l}0 \\ 0 \\ 1\end{array}\right)$ on the boundary with the second derivatives at a point $Q_{r}=\left(\begin{array}{l}r \\ 0 \\ 1\end{array}\right)$ near the boundary, by comparing the Taylor remainder estimates near $P$ and near $Q_{r}$. Let $T_{1}^{P} f$ denote the Taylor polynomial of $f$ at $P$ of degree 1 in both space and time, and let $T_{2,1}^{Q_{r}} f$ denote the Taylor polynomial of $f$ at $Q_{r}$ of degree 2 in space and 1 in time, and consider the remainders

$$
R_{1}^{P} f=f-T_{1}^{P} f \quad \text { and } \quad R_{2,1}^{Q_{r}} f=f-T_{2,1}^{Q_{r}} f .
$$

For $\lambda$ small the cylinder $\mathcal{A}_{\lambda r}\left(Q_{r}\right)$ is entirely contained in the box $\mathcal{B}_{2 r}(P)$. Our remainder estimate at the boundary gives

$$
\left\|R_{1}^{P} f\right\|_{C^{0}\left(\mathcal{B}_{2 r}(P)\right)} \leq C r^{1+\alpha}\left(\|f\|_{C^{0}\left(\mathcal{B}_{1}(P)\right)}+\left\|L_{0} f\right\|_{C_{s}^{\beta}\left(\mathcal{B}_{1}(P)\right)}\right)
$$

when $0<\beta<1$ and $\alpha=\beta / 2$ and $r \leq 1 / 2$. Let $\bar{f}=R_{1}^{P} f$, and apply the interior remainder estimate to $\bar{f}$ in Corollary I.8.8 to get

$$
\left\|R_{2,1}^{Q_{r} \bar{f}}\right\|_{C^{0}\left(\mathcal{A}_{\mu r}\left(Q_{r}\right)\right)} \leq C\left(\|\bar{f}\|_{C^{0}\left(\mathcal{A}_{\lambda r}\left(Q_{r}\right)\right)}+r^{1+\alpha}\left\|L_{0} \bar{f}\right\|_{H_{s}^{\beta}\left(\mathcal{A}_{\lambda r}\left(Q_{r}\right)\right)}\right) .
$$

Now any polynomial of degree $k$ is its own Taylor polynomial of degree $k$ at every point, so

$$
T_{2,1}^{Q_{r}} T_{1}^{P} f=T_{1}^{P} f
$$


and hence

$$
R_{2,1}^{Q_{r}} \bar{f}=R_{2,1}^{Q_{r}} R_{1}^{P} f=R_{2,1}^{Q_{r}} f .
$$

Since $\mathcal{A}_{\lambda r}\left(Q_{r}\right) \subseteq \mathcal{B}_{2 r}(P)$ we can estimate the $C^{0}$ norm of $\bar{f}$ by the boundary remainder estimate given before. Since $\bar{f}$ differs from $f$ by a polynomial of degree 1 in both space and time, $L_{0} \bar{f}$ differs from $L_{0} f$ by only a constant; and this constant is annihilated by the semi-norm $H^{\alpha}$. Thus

$$
\left\|L_{0} \bar{f}\right\|_{H_{s}^{\beta}\left(\mathcal{A}_{\lambda r}\left(Q_{r}\right)\right)}=\left\|L_{0} f\right\|_{H_{s}^{\beta}\left(\mathcal{A}_{\lambda r}\left(Q_{r}\right)\right)} .
$$

Combining these estimates gives

$$
\left\|R_{2,1}^{Q_{r}} f\right\|_{C^{0}\left(\mathcal{A}_{\mu r}\left(Q_{r}\right)\right)} \leq C r^{1+\alpha}\left(\|f\|_{C^{0}\left(\mathcal{B}_{1}(P)\right)}+\left\|L_{0} f\right\|_{C_{s}^{\beta}\left(\mathcal{B}_{1}(P)\right)}\right) .
$$

Now note that

$$
T_{2,1}^{Q_{r}} f-T_{1}^{P} f=R_{1}^{P} f-R_{2,1}^{Q_{r}} f
$$

and

$$
\left\|R_{1}^{P} f-R_{2,1}^{Q_{r}} f\right\|_{C^{0}\left(\mathcal{A}_{\mu r}\left(Q_{r}\right)\right)} \leq\left\|R_{1}^{P} f\right\|_{C^{0}\left(\mathcal{B}_{2 r}(P)\right)}+\left\|R_{2,1}^{Q_{r}} f\right\|_{C^{0}\left(\mathcal{A}_{\mu r}\left(Q_{r}\right)\right)}
$$

so

$$
\left\|T_{2,1}^{Q_{r}} f-T_{1}^{P} f\right\|_{C^{0}\left(\mathcal{A}_{\mu r}\left(Q_{r}\right)\right)} \leq C r^{1+\alpha}\left(\|f\|_{C^{0}\left(\mathcal{B}_{1}(P)\right)}+\left\|L_{0} f\right\|_{C_{s}^{\beta}\left(\mathcal{B}_{1}(P)\right)}\right) .
$$

Now it is possible to bound the coefficients of a polynomial from its supremum over a cylinder of a certain size, and hence to bound the derivatives of the polynomial. Consider the polynomial

$$
p=T_{2,1}^{Q_{r}} f-T_{1}^{P} f
$$

which is bounded on the cylinder $\mathcal{A}_{\mu r}\left(Q_{r}\right)$, and consider for example its second derivative $f_{x x}$ with respect to $x$ twice, which is a constant. We have the estimate

$$
\left|p_{x x}\right| \leq \frac{C}{r^{2}}\|p\|_{C^{0}\left(\mathcal{A}_{\mu r}\left(Q_{r}\right)\right)} .
$$

But

$$
\left(T_{2,1}^{Q_{r}} f\right)_{x x}=f_{x x}\left(Q_{r}\right)
$$

and

$$
\left(T_{1}^{P} f\right)_{x x}=0
$$

so

$$
p_{x x}=f_{x x}\left(Q_{r}\right) .
$$

This gives an estimate on $f_{x x}\left(Q_{r}\right)$, and the same applies to $f_{x y}\left(Q_{r}\right)$ and $f_{y y}\left(Q_{r}\right)$. This gives the following result.

I.9.1. Theorem. For any $\nu>0$ and any $\beta$ in $0<\beta<1$ we can find a constant $C$ with the following property. If $f$ is smooth in the box $\mathcal{B}_{1}$ and if $0<r \leq 1 / 2$ and if

$$
Q_{r}=\{x=r, y=0, t=1\}
$$

and $\alpha=\beta / 2$, then

$$
\left|f_{x x}\left(Q_{r}\right)\right|+\left|f_{x y}\left(Q_{r}\right)\right|+\left|f_{y y}\left(Q_{r}\right)\right| \leq C r^{\alpha-1}\left(\|f\|_{C^{0}\left(\mathcal{B}_{1}\right)}+\left\|L_{0} f\right\|_{C_{s}^{\beta}\left(\mathcal{B}_{1}\right)}\right) .
$$


By scaling space and time around the point $P=\{x=0, y=0, t=1\}=Q_{0}$ and translating in $y$ and $t$, we can get a similar estimate at any point in the box $\mathcal{B}_{1 / 4}$, since we only need to shrink $\mathcal{B}_{1}$ by a factor of at most $1 / 2$, and then $r \leq 1 / 2$ turns into $x \leq 1 / 4$. When we note that

$$
s\left[\left(\begin{array}{l}
x \\
y \\
t
\end{array}\right),\left(\begin{array}{l}
0 \\
y \\
t
\end{array}\right)\right]=\sqrt{2 x}
$$

we get the following.

I.9.2. Corollary. We also have

$$
\left|f_{x x}\right|+\left|f_{x y}\right|+\left|f_{y y}\right| \leq C s\left[\left(\begin{array}{l}
x \\
y \\
t
\end{array}\right),\left(\begin{array}{l}
0 \\
y \\
t
\end{array}\right)\right]^{\beta-2}\left(\|f\|_{C^{0}\left(\mathcal{B}_{1}\right)}+\left\|L_{0} f\right\|_{C_{s}^{\beta}\left(\mathcal{B}_{1}\right)}\right)
$$

for all $\left(\begin{array}{l}x \\ y \\ t\end{array}\right)$ in $\mathcal{B}_{1 / 4}$

I.9.3. Corollary. We also have

$$
\left|x f_{x x}\right|+\left|x f_{x y}\right|+\left|x f_{y y}\right| \leq C s\left[\left(\begin{array}{l}
x \\
y \\
t
\end{array}\right),\left(\begin{array}{l}
0 \\
y \\
t
\end{array}\right)\right]^{\beta}\left(\|f\|_{C^{0}\left(\mathcal{B}_{1}\right)}+\left\|L_{0} f\right\|_{C_{s}^{\beta}\left(\mathcal{B}_{1}\right)}\right) .
$$

Proof. Because $2 x=s^{2}$. This is a Hölder estimate on $x f_{x x}, x f_{x y}, x f_{y y}$ for shifts in $x$ only, since they are 0 at $x=0$.

I.9.4. Corollary. We also have

$$
\begin{aligned}
\left|f_{x}\left(\begin{array}{l}
x \\
y \\
t
\end{array}\right)-f_{x}\left(\begin{array}{l}
0 \\
y \\
t
\end{array}\right)\right| & +\left|f_{y}\left(\begin{array}{l}
x \\
y \\
t
\end{array}\right)-f_{y}\left(\begin{array}{l}
0 \\
y \\
t
\end{array}\right)\right|+\left|f_{t}\left(\begin{array}{l}
x \\
y \\
t
\end{array}\right)-f_{t}\left(\begin{array}{l}
0 \\
y \\
t
\end{array}\right)\right| \\
& \leq C s\left[\left(\begin{array}{l}
x \\
y \\
t
\end{array}\right),\left(\begin{array}{l}
0 \\
y \\
t
\end{array}\right)\right]^{\beta}\left(\|f\|_{C^{0}\left(\mathcal{B}_{1}\right)}+\left\|L_{0} f\right\|_{C_{s}^{\beta}\left(\mathcal{B}_{1}\right)}\right) .
\end{aligned}
$$

Proof. We integrate

$$
f_{x}\left(\begin{array}{l}
x \\
y \\
t
\end{array}\right)-f_{x}\left(\begin{array}{l}
0 \\
y \\
t
\end{array}\right)=\int_{u=0}^{x} f_{x x}\left(\begin{array}{l}
u \\
y \\
t
\end{array}\right) d u
$$

and use

$$
\left|f_{x x}\left(\begin{array}{l}
u \\
y \\
t
\end{array}\right)\right| \leq C u^{\alpha-1}\left(\|f\|_{C^{0}\left(\mathcal{B}_{1}\right)}+\left\|L_{0} f\right\|_{C_{s}^{\beta}\left(\mathcal{B}_{1}\right)}\right)
$$

and

$$
\int_{u=0}^{x} u^{\alpha-1} d u=\frac{x^{\alpha}}{\alpha}
$$

to get the bound on $f_{x}$. The bound on $f_{y}$ follows by integrating $f_{x y}$ the same way. The bound on $f_{t}$ follows from the equation

$$
f_{t}=x\left(f_{x x}+f_{y y}\right)+\nu f_{x}+g
$$


where $g=L_{0} f$, and the Hölder bound on $g$ which is given. These are Hölder bounds on $f_{x}, f_{y}$ and $f_{t}$ for shifts in $x$ only.

We can also make Hölder estimates in the interior using the bound on $\bar{f}=R_{1}^{P} f$. As in the previous argument on the remainder formula we get

$$
\|\bar{f}\|_{H_{s}^{2+\beta}\left(\mathcal{A}_{\mu r}\left(Q_{r}\right)\right)} \leq C\left(\frac{1}{r^{1+\alpha}}\|\bar{f}\|_{C^{0}\left(\mathcal{A}_{\lambda r}\left(Q_{r}\right)\right)}+\left\|L_{0} \bar{f}\right\|_{H_{s}^{\beta}\left(\mathcal{A}_{\lambda r}\left(Q_{r}\right)\right)}\right)
$$

and $\mathcal{A}_{\lambda r}\left(Q_{r}\right) \subseteq \mathcal{B}_{2 r}(P)$ and

$$
\|\bar{f}\|_{C^{0}\left(\mathcal{B}_{2 r}(P)\right)} \leq C r^{1+\alpha}\left(\|f\|_{C^{0}\left(\mathcal{B}_{1}(P)\right)}+\left\|L_{0} f\right\|_{C_{s}^{\beta}\left(\mathcal{B}_{1}(P)\right)}\right)
$$

and

$$
\left\|L_{0} \bar{f}\right\|_{H_{s}^{\beta}\left(\mathcal{A}_{\lambda r}\left(Q_{r}\right)\right)} \leq C\left\|L_{0} f\right\|_{C_{s}^{\beta}\left(\mathcal{B}_{1}(P)\right)}
$$

We also have

$$
\|\bar{f}\|_{H_{s}^{2+\beta}}=\|f\|_{H_{s}^{2+\beta}}
$$

since the $H_{s}^{2+\beta}$ semi-norm measures the $H_{s}^{\beta}$ norm of first or second derivatives only, and first derivatives of the first order polynomial

$$
T_{1}^{P} f=f-\bar{f}
$$

are constant, and another difference or derivative gives zero. Combining these proves the following result.

I.9.5. Theorem. Under the hypotheses of Theorem I.9.1, we also have

$$
\|f\|_{H_{s}^{2+\beta}\left(\mathcal{A}_{\mu r}\left(Q_{r}\right)\right)} \leq C\left(\|f\|_{C^{0}\left(\mathcal{B}_{1}(P)\right)}+\left\|L_{0} f\right\|_{C_{s}^{\beta}\left(\mathcal{B}_{1}(P)\right)}\right) .
$$

I.9.6. Theorem. For all $\left(\begin{array}{l}x \\ y \\ t\end{array}\right)$ in $\mathcal{A}_{\mu r}\left(Q_{r}\right)$ we have

$$
\begin{aligned}
\left|f_{x}\left(\begin{array}{l}
x \\
y \\
t
\end{array}\right)-f_{x}\left(\begin{array}{l}
r \\
0 \\
1
\end{array}\right)\right| & +\left|f_{y}\left(\begin{array}{l}
x \\
y \\
t
\end{array}\right)-f_{y}\left(\begin{array}{l}
r \\
0 \\
1
\end{array}\right)\right|+\left|f_{t}\left(\begin{array}{l}
x \\
y \\
t
\end{array}\right)-f_{t}\left(\begin{array}{l}
r \\
0 \\
1
\end{array}\right)\right| \\
& +\left|x f_{x x}\left(\begin{array}{l}
x \\
y \\
t
\end{array}\right)-r f_{x x}\left(\begin{array}{l}
r \\
0 \\
1
\end{array}\right)\right|+\left|x f_{x y}\left(\begin{array}{l}
x \\
y \\
t
\end{array}\right)-r f_{x y}\left(\begin{array}{l}
r \\
0 \\
1
\end{array}\right)\right| \\
& +\left|x f_{y y}\left(\begin{array}{l}
x \\
y \\
t
\end{array}\right)-r f_{y y}\left(\begin{array}{l}
r \\
0 \\
1
\end{array}\right)\right| \\
& \leq C s\left[\left(\begin{array}{l}
x \\
y \\
t
\end{array}\right),\left(\begin{array}{l}
r \\
0 \\
1
\end{array}\right)\right]^{\beta}\left(\|f\|_{C^{0}\left(\mathcal{B}_{1}\right)}+\left\|L_{0} f\right\|_{C_{s}^{\beta}\left(\mathcal{B}_{1}\right)}\right) .
\end{aligned}
$$

I.9.7. Corollary. For any $\nu>0$ and any $\beta$ in $0<\beta<1$ we can find $\varepsilon>0$ and a constant $C<\infty$ with the following property. If $f$ is a smooth function on $\mathcal{B}_{1}$ and if

$$
P_{1}=\left(\begin{array}{l}
x_{1} \\
y_{1} \\
t_{1}
\end{array}\right) \quad \text { and } \quad P_{2}=\left(\begin{array}{l}
x_{2} \\
y_{2} \\
t_{2}
\end{array}\right)
$$


are any two points in $\mathcal{B}_{1 / 4}$ and if

$$
\left|x_{1}-x_{2}\right|+\left|y_{1}-y_{2}\right|+\left|t_{1}-t_{2}\right| \leq \varepsilon\left(x_{1}+x_{2}\right),
$$

then

$$
\begin{aligned}
\left|x f_{x x}\left(P_{1}\right)-x f_{x x}\left(P_{2}\right)\right| & +\left|x f_{x y}\left(P_{1}\right)-x f_{x y}\left(P_{2}\right)\right|+\left|x f_{y y}\left(P_{1}\right)-x f_{y y}\left(P_{2}\right)\right| \\
& \leq C s\left(P_{1}, P_{2}\right)^{\beta}\left(\|f\|_{C^{0}\left(B_{1}\right)}+\left\|L_{0} f\right\|_{C_{s}^{\beta}\left(B_{1}\right)}\right) .
\end{aligned}
$$

Proof. Assume $t_{2} \geq t_{1}$ (or else switch). Translate $y$ and $t$ so that $y_{2}=0$ and $t_{2}=1$, and then dilate $x, y, t$ by a factor $1 /\left(1-t_{2}\right) \leq 2$ so that the new function is still defined on $B_{1}$. Now $P_{2}=\left(\begin{array}{l}r \\ 0 \\ 1\end{array}\right)$ for some $r \leq 1 / 2$, and if $\varepsilon>0$ is small enough the new point $P_{1}$ will lie in $\mathcal{A}_{\mu r}\left(Q_{r}\right)$ when $P_{2}=Q_{r}$ now. We can then apply the previous result, and the constant will have changed by a bounded factor.

I.9.8. Theorem. For any $\nu>0$ and any $\beta$ in $0<\beta<1$ we can find $\delta>0$ and a constant $C<\infty$ such that if $P_{1}$ and $P_{2}$ are any two points in the box $\mathcal{B}_{\delta}$ and if $f$ is any smooth function on the box $\mathcal{B}_{1}$, then

$$
\begin{aligned}
\left|f_{x}\left(P_{1}\right)-f_{x}\left(P_{2}\right)\right| & +\left|f_{y}\left(P_{1}\right)-f_{y}\left(P_{2}\right)\right|+\left|f_{t}\left(P_{1}\right)-f_{t}\left(P_{2}\right)\right| \\
& +\left|x f_{x x}\left(P_{1}\right)-x f_{x x}\left(P_{2}\right)\right|+\left|x f_{x y}\left(P_{1}\right)-x f_{x y}\left(P_{2}\right)\right| \\
& +\left|x f_{y y}\left(P_{1}\right)-x f_{y y}\left(P_{2}\right)\right| \\
& \leq C s\left(P_{1}, P_{2}\right)^{\beta}\left(\|f\|_{C^{0}\left(\mathcal{B}_{1}\right)}+\left\|L_{0} f\right\|_{C_{s}^{\beta}\left(\mathcal{B}_{1}\right)}\right) .
\end{aligned}
$$

Proof. By the previous result we are done with $\delta \leq 1 / 4$ unless

$$
\left|x_{1}-x_{2}\right|+\left|y_{1}-y_{2}\right|+\left|t_{1}-t_{2}\right| \geq \varepsilon\left(x_{1}+x_{2}\right)
$$

for some $\varepsilon>0$. In this case the metric distance

$$
s\left(P_{1}, P_{2}\right) \approx \sqrt{\left|x_{1}-x_{2}\right|}+\sqrt{\left|y_{1}-y_{2}\right|}+\sqrt{\left|t_{1}-t_{2}\right|}
$$

in the sense of being bounded above and below by a constant times the expression. Choose

$$
r=B s\left(P_{1}, P_{2}\right)^{2}
$$

where $B$ is a large constant so that

$$
\left|y_{1}-y_{2}\right|+\left|t_{1}-t_{2}\right| \leq \varepsilon r
$$

and where $\delta$ is so small that $r \leq 1 / 2$ for all $P_{1}$ and $P_{2}$ in $\mathcal{B}_{\delta}$ for this $B$. Let

$$
P_{3}=\left(\begin{array}{c}
0 \\
y_{1} \\
t_{1}
\end{array}\right), \quad P_{4}=\left(\begin{array}{c}
0 \\
y_{2} \\
t_{2}
\end{array}\right), \quad P_{5}=\left(\begin{array}{c}
r \\
y_{1} \\
t_{1}
\end{array}\right), \quad P_{6}=\left(\begin{array}{c}
r \\
y_{2} \\
t_{2}
\end{array}\right) \text {. }
$$

Our strategy is to compare a function, say $f_{x}$ or $x f_{x x}$, at $P_{1}$ with its value at $P_{3}$, and that with its value at $P_{5}$; likewise its value at $P_{2}$ with its value at $P_{4}$, and that with its value at $P_{6}$; and finally the value at $P_{5}$ with the value at $P_{6}$. We can bound all these distances in terms of $r$

$$
s\left(P_{1}, P_{3}\right)+s\left(P_{3}, P_{5}\right)+s\left(P_{2}, P_{4}\right)+s\left(P_{4}, P_{6}\right)+s\left(P_{5}, P_{6}\right) \leq C \sqrt{r}
$$

and $\sqrt{r} \leq B s\left(P_{1}, P_{2}\right)$. The comparisons of the function between $P_{1}$ and $P_{3}$ or $P_{3}$ and $P_{5}$ or $P_{2}$ and $P_{4}$ or $P_{4}$ and $P_{6}$ now follow from Corollary I.9.3, while that between $P_{5}$ and $P_{6}$ follows from Corollary I.9.7. Note the path $P_{3} P_{1} P_{5} P_{6} P_{4} P_{2}$ 
somewhat resembles the cycloid, which is a geodesic in our metric. This gave rise to this idea.

Now it is possible to bound the $C_{s}^{2+\beta}$ norm of $f$ using the $H_{s}^{2+\beta}$ norm and the $C^{0}$ norm. For example, suppose we wish to bound the $C^{0}$ norm of $f_{x}$. Let its maximum $M$ over $\mathcal{B}_{\delta}$ occur at a point $\left(\begin{array}{c}x_{0} \\ y_{0} \\ t_{0}\end{array}\right)$ where

$$
\left|f_{x}\left(\begin{array}{c}
x_{0} \\
y_{0} \\
t_{0}
\end{array}\right)\right|=M \text {. }
$$

We know for all $\left(\begin{array}{l}x \\ y \\ t\end{array}\right)$ in $\mathcal{B}_{\delta}$ that

$$
\left|f_{x}\left(\begin{array}{l}
x \\
y \\
t
\end{array}\right)-f_{x}\left(\begin{array}{l}
x_{0} \\
y_{0} \\
t_{0}
\end{array}\right)\right| \leq C\left(\|f\|_{C^{0}\left(\mathcal{B}_{1}\right)}+\left\|L_{0} f\right\|_{C_{s}^{\beta}\left(\mathcal{B}_{1}\right)}\right) .
$$

Unless

$$
M \leq 2 C\left(\|f\|_{C^{0}\left(\mathcal{B}_{1}\right)}+\left\|L_{0} f\right\|_{C_{s}^{\beta}\left(\mathcal{B}_{1}\right)}\right)
$$

we have

$$
\left|f_{x}\left(\begin{array}{l}
x \\
y \\
t
\end{array}\right)\right| \geq M / 2
$$

on all of $\mathcal{B}_{\delta}$. Integrating in $x$ makes

$$
\left|f\left(\begin{array}{c}
x \\
y_{0} \\
t_{0}
\end{array}\right)-f\left(\begin{array}{c}
x_{0} \\
y_{0} \\
t_{0}
\end{array}\right)\right| \geq \frac{M}{2}\left|x-x_{0}\right|
$$

and since $\left|x-x_{0}\right| \geq \delta / 2$ for some $x$ we get a contradiction if $f$ is bounded and $M$ is too big. The other bounds follow in the same way. For example, the bound on $x f_{x x}$ follows from

$$
x f_{x x}=\left(x f_{x}-f\right)_{x} .
$$

If $x f_{x x}$ is too large at one point, then $x f_{x}-f$ has too large a variation.

I.9.9. Corollary. We also have

$$
\|f\|_{C_{s}^{2+\beta}\left(\mathcal{B}_{\delta}\right)} \leq C\left(\|f\|_{C^{0}\left(\mathcal{B}_{1}\right)}+\left\|L_{0} f\right\|_{C_{s}^{\beta}\left(\mathcal{B}_{1}\right)}\right) .
$$

I.10. Main Schauder estimate. Combining the results in the previous sections we can prove now our main Schauder estimate.

I.10.1. Theorem (Schauder Estimate). For any $\nu>0$, any $\alpha$ in $0<\alpha<1$ and any $r<1$ there is a constant $C$ so that

$$
\|f\|_{C_{s}^{2+\alpha}\left(\mathcal{B}_{r}\right)} \leq C\left(\|f\|_{C_{s}^{\circ}\left(\mathcal{B}_{1}\right)}+\left\|L_{0} f\right\|_{C_{s}^{\alpha}\left(\mathcal{B}_{1}\right)}\right)
$$

for all $C^{\infty}$ smooth functions $f$ on $\mathcal{B}_{1}$.

Proof. The result follows directly; since for any $r<1$ we can cover a neighborhood of the part of the box $\mathcal{B}_{r}$ along the boundary $\{x=0\}$ with little boxes that translate and dilate to $\mathcal{B}_{\delta}$ as before. Having the estimate in a neighborhood of the boundary reduces it to an interior problem, which we can handle in the same manner. 
The next Corollary can be shown via standard rescaling.

I.10.2. Corollary. For any $\nu>0$, any $\alpha$ in $0<\alpha<1$ and any $0<r<\rho<1$ there is a constant $C$ so that

$$
\|f\|_{C_{s}^{2+\alpha}\left(\mathcal{B}_{r}\right)} \leq C\left(\|f\|_{C_{s}^{\circ}\left(\mathcal{B}_{\rho}\right)}+\left\|L_{0} f\right\|_{C_{s}^{\alpha}\left(\mathcal{B}_{\rho}\right)}\right)
$$

for all $C^{\infty}$ smooth functions $f$ on $\mathcal{B}_{1}$.

We will now give the proof of Theorem I.1.3.

Proof of Theorem I.1.3. Assume first that $k=1$. Let $f$ be a smooth function in the box $\mathcal{B}_{1}$ and set $L_{0} f=g$. The derivatives $f_{t}, f_{x}$ and $f_{y}$ satisfy the equations

$$
\begin{aligned}
& \left(f_{t}\right)_{t}-x\left[\left(f_{t}\right)_{x x}+\left(f_{t}\right)_{y y}\right]-\nu\left(f_{t}\right)_{x}=g_{t}, \\
& \left(f_{y}\right)_{t}-x\left[\left(f_{y}\right)_{x x}+\left(f_{y}\right)_{y y}\right]-\nu\left(f_{y}\right)_{x}=g_{y}, \\
& \left(f_{x}\right)_{t}-x\left[\left(f_{x}\right)_{x x}+\left(f_{x}\right)_{y y}\right]-(\nu+1)\left(f_{x}\right)_{x}=g_{x}+f_{y y}
\end{aligned}
$$

in the box $\mathcal{B}_{1}$. For any $r<1$, set $\rho_{1}=(1+2 r) / 3$ and $\rho_{2}=(2+r) / 3$. Then $0<r<\rho_{1}<\rho_{2}<1$. From the estimates in Theorem I.10.1 and Corollary I.10.2 we have

$$
\|f\|_{C_{s}^{2+\alpha}\left(\mathcal{B}_{\rho_{2}}\right)} \leq C\left(\|f\|_{C_{s}^{\circ}\left(\mathcal{B}_{1}\right)}+\|g\|_{C_{s}^{\alpha}\left(\mathcal{B}_{1}\right)}\right)
$$

and

$$
\left\|f_{t}\right\|_{C_{s}^{2+\alpha}\left(\mathcal{B}_{\rho_{1}}\right)}+\left\|f_{y}\right\|_{C_{s}^{2+\alpha}\left(\mathcal{B}_{\rho_{1}}\right)} \leq C\left(\|D f\|_{C_{s}^{\circ}\left(\mathcal{B}_{\rho_{2}}\right)}+\|D g\|_{C_{s}^{\alpha}\left(\mathcal{B}_{\rho_{2}}\right)}\right)
$$

and

$$
\left\|f_{x}\right\|_{C_{s}^{2+\alpha}\left(\mathcal{B}_{r}\right)} \leq C\left(\|D f\|_{C_{s}^{\circ}\left(\mathcal{B}_{\rho_{1}}\right)}+\|D g\|_{C_{s}^{\alpha}\left(\mathcal{B}_{\rho_{1}}\right)}+\left\|f_{y y}\right\|_{C_{s}^{\alpha}\left(\mathcal{B}_{\rho_{1}}\right)}\right) .
$$

Combining the estimates above we conclude

$$
\|f\|_{C_{s}^{1,2+\alpha}\left(\mathcal{B}_{r}\right)} \leq C\left(\|f\|_{C_{s}^{\circ}\left(\mathcal{B}_{1}\right)}+\|g\|_{C_{s}^{1, \alpha}\left(\mathcal{B}_{1}\right)}\right)
$$

which is the desired estimate for $k=1$. The same proof, with a bit more involved notation, generalizes for all $k \geq 1$. The constant $C$ in this case depends on the integer $k$.

I.11. Smoothing operators and an Extension Lemma. The proof of Theorem I.1.1 will follow from Theorems I.1.2 and I.1.3 via a regularizing argument which will involve appropriate smoothing operators with respect to the metric $s$. We begin by defining these operators.

Let $P=\left(\begin{array}{l}x \\ y\end{array}\right)$ be a point on the half-space $x \geq 0$ and $Q=\left(\begin{array}{l}u \\ v\end{array}\right)$ any point in the unit box

$$
\mathcal{B}_{1}=\{|u|<1,|v| \leq 1\}
$$

For $\epsilon>0$ we define the point $M_{\epsilon}(P ; Q)$ as follows. Starting from the point $\left(\begin{array}{c}x+2 \epsilon \\ y\end{array}\right)$ we first move by a distance $\sqrt{\epsilon}|u|$ (in the $s$ metric) in the direction parallel to the $x$-axis and to the right or left of $\left(\begin{array}{c}x+2 \epsilon \\ y\end{array}\right)$ if $u>0$ or $u<0$ respectively. Then, starting from the new point we move by a distance $\sqrt{\epsilon}|v|$ (again in the $s$ metric) in the direction of the $y$-axis and above or below of the new point if $v>0$ or $v<0$ respectively. Thus if the point $M_{\epsilon}(P ; Q)$ has coordinates

$$
M_{\epsilon}(P ; Q)=\left(\begin{array}{l}
\xi \\
\zeta
\end{array}\right)
$$


we have

$$
s\left[\left(\begin{array}{c}
x+2 \epsilon \\
y
\end{array}\right),\left(\begin{array}{l}
\xi \\
y
\end{array}\right)\right]=\sqrt{\epsilon}|u|, \quad s\left[\left(\begin{array}{l}
\xi \\
y
\end{array}\right),\left(\begin{array}{l}
\xi \\
\zeta
\end{array}\right)\right]=\sqrt{\epsilon}|v|
$$

where

$$
\xi \geq x+2 \epsilon \quad \text { iff } \quad u \geq 0 \quad \text { and } \quad \zeta \geq y \quad \text { iff } \quad v \geq 0 .
$$

Since for $d y=0, d s=d x /(\sqrt{2 x})$ and for $d x=0, d s=d y /(\sqrt{2 x})$, we compute

$$
\left\{\begin{array}{l}
\sqrt{2 \xi}-\sqrt{2(x+2 \epsilon)}=\sqrt{\epsilon} u \\
\zeta-y=\sqrt{2 \xi} \sqrt{\epsilon} v
\end{array}\right.
$$

Let $\varphi$ be a standard smooth, nonnegative bump function, supported in the box $\mathcal{B}_{1}$, with

$$
\int \varphi(u, v) d u d v=1
$$

and let $h=h\left(\begin{array}{l}x \\ y\end{array}\right)$ be a function defined on the half-space $S_{0}$ where $x \geq 0$. We define the spatial regularizations $h_{\epsilon}$ of $h$ by

$$
h_{\epsilon}(P)=\int \varphi\left(\begin{array}{c}
u \\
v
\end{array}\right) h\left(M_{\epsilon}\left(P ;\left(\begin{array}{l}
u \\
v
\end{array}\right)\right)\right) d u d v=\int \varphi\left(M_{\epsilon}^{-1}\left(P ;\left(\begin{array}{l}
\xi \\
\zeta
\end{array}\right)\right)\right) h\left(\begin{array}{l}
\xi \\
\zeta
\end{array}\right) \frac{d \xi}{2 \epsilon \xi} d \zeta
$$

for $P=\left(\begin{array}{l}x \\ y\end{array}\right) \in S_{0}$. We begin with the following useful Lemma.

I.11.2. Lemma. For any two points $P=\left(\begin{array}{l}x \\ y\end{array}\right)$ and $P^{\prime}=\left(\begin{array}{l}x^{\prime} \\ y^{\prime}\end{array}\right)$ in $\mathcal{S}_{0}$ and any point $Q=\left(\begin{array}{l}u \\ v\end{array}\right)$ in $B_{1}$, we have

$$
s\left[M_{\epsilon}(P ; Q), M_{\epsilon}\left(P^{\prime} ; Q\right)\right] \leq C s\left[P, P^{\prime}\right]
$$

and

$$
s\left[M_{\epsilon}(P ; Q), P\right] \leq 4 \sqrt{\epsilon}
$$

with the constant $C$ independent of $\epsilon$.

Proof. Let $M_{\epsilon}(P ; Q)=\left(\begin{array}{l}\xi \\ \zeta\end{array}\right)$ and $M_{\epsilon}\left(P^{\prime} ; Q\right)=\left(\begin{array}{l}\xi^{\prime} \\ \zeta^{\prime}\end{array}\right)$. We can assume that $x^{\prime} \geq x$. If $x=x^{\prime}$, then by (I.11.1) $\xi=\xi^{\prime}$ and $\zeta-y=\zeta^{\prime}-y^{\prime}$. Therefore, since on $|u| \leq 1$ we have

$$
\sqrt{2 \xi} \geq \sqrt{2(x+2 \epsilon)}-\sqrt{\epsilon} \geq \sqrt{x}
$$

and for $d \xi=0, d s=d \zeta / \sqrt{2 \xi}$, we estimate

$$
s\left[\left(\begin{array}{c}
\xi \\
\zeta
\end{array}\right) ;\left(\begin{array}{c}
\xi^{\prime} \\
\zeta^{\prime}
\end{array}\right)\right] \leq \frac{1}{\sqrt{2 \xi}}\left|\zeta-\zeta^{\prime}\right| \leq \frac{1}{\sqrt{x}}\left|y-y^{\prime}\right|=\sqrt{2} s\left[\left(\begin{array}{c}
x \\
y
\end{array}\right) ;\left(\begin{array}{l}
x^{\prime} \\
y^{\prime}
\end{array}\right)\right] .
$$

On the other hand, if $y=y^{\prime}$, then, by (I.11.1), we have

$$
\begin{aligned}
\left|\zeta-\zeta^{\prime}\right| & =\sqrt{\epsilon} v\left|\sqrt{2 \xi}-\sqrt{2 \xi^{\prime}}\right|=\sqrt{\epsilon} v\left|\sqrt{2(x+2 \epsilon)}-\sqrt{2\left(x^{\prime}+2 \epsilon\right)}\right| \\
& \leq \sqrt{\epsilon} v\left|\sqrt{2 x}-\sqrt{2 x^{\prime}}\right|
\end{aligned}
$$

since the function $\psi(\epsilon)=\sqrt{2(x+2 \epsilon)}-\sqrt{2\left(x^{\prime}+2 \epsilon\right)}$ is decreasing in $\epsilon$. Thus, since

$$
s\left[\left(\begin{array}{c}
\xi \\
\zeta
\end{array}\right) ;\left(\begin{array}{c}
\xi^{\prime} \\
\zeta^{\prime}
\end{array}\right)\right] \leq C \frac{\left|\xi-\xi^{\prime}\right|+\left|\zeta-\zeta^{\prime}\right|}{\sqrt{\xi}+\sqrt{\xi^{\prime}}+\sqrt{\left|\zeta-\zeta^{\prime}\right|}} \leq C\left(\left|\sqrt{\xi}-\sqrt{\xi^{\prime}}\right|+\frac{\left|\zeta-\zeta^{\prime}\right|}{\sqrt{\xi^{\prime}}}\right)
$$


we estimate

$$
s\left[\left(\begin{array}{c}
\xi \\
\zeta
\end{array}\right) ;\left(\begin{array}{c}
\xi^{\prime} \\
\zeta^{\prime}
\end{array}\right)\right] \leq C\left|\sqrt{2 x}-\sqrt{2 x^{\prime}}\right|\left(1+\frac{\sqrt{\epsilon} v}{\sqrt{2 \xi^{\prime}}}\right) \leq C s\left[\left(\begin{array}{l}
x \\
y
\end{array}\right) ;\left(\begin{array}{l}
x^{\prime} \\
y^{\prime}
\end{array}\right)\right]
$$

since $\sqrt{\xi^{\prime}} \geq \sqrt{\epsilon}$. Since

$$
s\left[\left(\begin{array}{l}
x \\
y
\end{array}\right),\left(\begin{array}{l}
x^{\prime} \\
y^{\prime}
\end{array}\right)\right] \leq s\left[\left(\begin{array}{l}
x \\
y
\end{array}\right),\left(\begin{array}{c}
x^{\prime} \\
y
\end{array}\right)\right]+s\left[\left(\begin{array}{c}
x^{\prime} \\
y
\end{array}\right),\left(\begin{array}{l}
x^{\prime} \\
y^{\prime}
\end{array}\right)\right] \leq c s\left[\left(\begin{array}{l}
x \\
y
\end{array}\right),\left(\begin{array}{l}
x^{\prime} \\
y^{\prime}
\end{array}\right)\right]
$$

for some $c>0$, the first estimate follows. As for the second estimate, we compute

$$
\begin{aligned}
s\left[M_{\epsilon}(P ; Q), P\right] & \leq s\left[M_{\epsilon}(P ; Q),\left(\begin{array}{c}
x+2 \epsilon \\
y
\end{array}\right)\right]+s\left[\left(\begin{array}{c}
x+2 \epsilon \\
y
\end{array}\right) ; P\right] \\
& \leq 2 \sqrt{\epsilon}+\sqrt{2(x+2 \epsilon)}-\sqrt{2 x} \leq 4 \sqrt{\epsilon}
\end{aligned}
$$

because by definition $s\left[M_{\epsilon}(P ; Q),\left(\begin{array}{c}x+2 \epsilon \\ y\end{array}\right)\right]=2 \sqrt{\epsilon}$ and $\psi(x)=\sqrt{2(x+2 \epsilon)}-\sqrt{2 x}$ is decreasing in $x$.

We can now give our regularization result in the metric $s$.

I.11.3. Theorem. If $h \in \mathcal{C}_{s}^{\alpha}\left(\mathcal{S}_{0}\right)$, then $h_{\epsilon}$ is smooth on $\mathcal{S}_{0}$,

$$
\left\|h_{\epsilon}\right\|_{\mathcal{C}_{s}^{\alpha}\left(\mathcal{S}_{0}\right)} \leq C\|h\|_{\mathcal{C}_{s}^{\alpha}\left(\mathcal{S}_{0}\right)}
$$

and for all points $\left(\begin{array}{l}x \\ y\end{array}\right)$ in $S_{0}$

$$
\left|h_{\epsilon}\left(\begin{array}{l}
x \\
y
\end{array}\right)-h\left(\begin{array}{l}
x \\
y
\end{array}\right)\right| \leq C \epsilon^{\alpha / 2}\|h\|_{\mathcal{C}_{s}^{\alpha}\left(\mathcal{S}_{0}\right)} .
$$

Therefore $h_{\epsilon} \rightarrow h$, uniformly on $S_{0}$.

Proof. We first notice that the point $M_{\epsilon}(P ; Q)=\left(\begin{array}{l}\xi \\ \zeta\end{array}\right)$, with $P \in S_{0}$ and $Q \in B_{1}$ has $\sqrt{2 \xi} \geq \sqrt{\epsilon}$. Hence, each function $h_{\epsilon}$ is smooth on $\mathcal{S}_{0}$. It is clear that $\left\|h_{\epsilon}\right\|_{\mathcal{C}_{0}} \leq\|h\|_{\mathcal{C}_{0}}$. Also, by the previous lemma, for $P, P^{\prime} \in S_{0}$ we have

$$
\left|h_{\epsilon}(P)-h_{\epsilon}\left(P^{\prime}\right)\right| \leq \int \varphi(Q)\left|h\left(M_{\epsilon}(P ; Q)\right)-h\left(M_{\epsilon}\left(P^{\prime} ; Q\right)\right)\right| \leq C\|h\|_{\mathcal{C}_{s}^{\alpha}\left(\mathcal{S}_{0}\right)} s\left[P ; P^{\prime}\right]^{\alpha}
$$

since $\int \varphi=1$. Thus, $\left\|h_{\epsilon}\right\|_{\mathcal{C}_{s}^{\alpha}\left(\mathcal{S}_{0}\right)} \leq C\|h\|_{\mathcal{C}_{s}^{\alpha}\left(\mathcal{S}_{0}\right)}$, for some constant $C$ independent of $\epsilon$. Moreover, for any $P \in S_{0}$ we have

$$
\left|h_{\epsilon}(P)-h(P)\right| \leq \int \varphi(Q)\left|h\left(M_{\epsilon}(P ; Q)\right)-h(P)\right| \leq\|h\|_{\mathcal{C}_{s}^{\alpha}\left(\mathcal{S}_{0}\right)} s\left[M_{\epsilon}(P ; Q), P\right]^{\alpha}
$$

with $s\left[M_{\epsilon}(P ; Q), P\right] \leq 4 \sqrt{\epsilon}$, as we proved in Lemma I.11.2. Therefore $h_{\epsilon} \rightarrow h$, uniformly on $S_{0}$.

We continue with an Extension Lemma on the new Hölder spaces. Such a result is standard for regular Hölder spaces. We denote, as usual by $\mathcal{S}$ the space $\mathcal{S}_{0} \times[0, \infty)$.

I.11.4. Theorem. Assume that $g \in \mathcal{C}_{s}^{\alpha}(\mathcal{S})$ and $f^{0} \in \mathcal{C}_{s}^{2+\alpha}\left(\mathcal{S}_{0}\right)$, for some number $\alpha$ in $0<\alpha<1$. Then, there exists a function $h \in \mathcal{C}_{s}^{2+\alpha}(\mathcal{S})$ such that

$$
h\left(\begin{array}{l}
x \\
y \\
0
\end{array}\right)=f^{0}\left(\begin{array}{l}
x \\
y
\end{array}\right) \quad \text { and } \quad \frac{\partial h}{\partial t}\left(\begin{array}{l}
x \\
y \\
0
\end{array}\right)=g\left(\begin{array}{l}
x \\
y \\
0
\end{array}\right)
$$

and

$$
\|h\|_{\mathcal{C}_{s}^{2+\alpha}(\mathcal{S})} \leq C\left(\left\|f^{0}\right\|_{\mathcal{C}_{s}^{2+\alpha}\left(\mathcal{S}_{0}\right)}+\|g\|_{\mathcal{C}_{s}^{\alpha}(\mathcal{S})}\right)
$$

for some constant $C$ depending only on $\alpha$. 
Proof. The result can be easily reduced to the case that $f^{0} \equiv 0$ in $\mathcal{S}_{0}$. We will show that in this case the function

$$
h\left(\begin{array}{l}
x \\
y \\
t
\end{array}\right)=\int_{0}^{t} d \tau \int \varphi\left(\begin{array}{l}
u \\
v
\end{array}\right) g\left(M_{\tau}\left(\left(\begin{array}{l}
x \\
y
\end{array}\right) ;\left(\begin{array}{l}
u \\
v
\end{array}\right)\right)\right) d u d v
$$

defined on $\mathcal{S}$ has the desired properties. It is easy to show that

$$
h\left(\begin{array}{l}
x \\
y \\
0
\end{array}\right)=0 \quad \text { and } \quad \frac{\partial h}{\partial t}\left(\begin{array}{l}
x \\
y \\
0
\end{array}\right)=g\left(\begin{array}{l}
x \\
y \\
0
\end{array}\right)
$$

since $g$ is a continuous function. Hence, it remains to show that $h$ belongs to the space $\mathcal{C}_{s}^{2+\alpha}(\mathcal{S})$ and satisfies

$$
\|h\|_{\mathcal{C}_{s}^{2+\alpha}(\mathcal{S})} \leq C\|g\|_{\mathcal{C}_{s}^{\alpha}(\mathcal{S})} .
$$

The proof of such an estimate is standard for Hölder spaces in the Euclidean metric. The calculations in our Hölder spaces are a bit more involved but similar. We will only show that $x h_{x x}$ belongs in the space $\mathcal{C}_{s}^{\alpha}(\mathcal{S})$ and satisfies the estimate

$$
\left\|x h_{x x}\right\|_{\mathcal{C}_{s}^{\alpha}(\mathcal{S})} \leq C\|g\|_{\mathcal{C}_{s}^{\alpha}(\mathcal{S})} \text {. }
$$

All the other estimates can be derived in a similar manner.

We first estimate the $L^{\infty}$ norm of $x h_{x x}$. Changing variables we can write $h$ as

$$
h\left(\begin{array}{l}
x \\
y \\
t
\end{array}\right)=\int_{0}^{t} d \tau \int \varphi\left(M_{\tau}^{-1}\left(\left(\begin{array}{l}
x \\
y
\end{array}\right) ;\left(\begin{array}{l}
\xi \\
\zeta
\end{array}\right)\right)\right) g\left(\begin{array}{l}
\xi \\
\zeta
\end{array}\right) \frac{d \xi}{2 \tau \xi} d \zeta .
$$

If we set

$$
M_{\tau}^{-1}\left(\left(\begin{array}{l}
x \\
y
\end{array}\right) ;\left(\begin{array}{l}
\xi \\
\zeta
\end{array}\right)\right)=\left(\begin{array}{l}
u \\
v
\end{array}\right)
$$

then by (I.11.1) we have

$$
u=\frac{\sqrt{2 \xi}-\sqrt{2(x+2 \tau)}}{\sqrt{\tau}} \quad \text { and } \quad v=\frac{\zeta-y}{\sqrt{2 \xi} \sqrt{\tau}}
$$

and therefore find that

$$
x h_{x x}=\int_{0}^{t} d \tau \int\left[\frac{x}{2 \tau(x+2 \tau)} \varphi_{u u}+\frac{x}{2 \sqrt{2 \tau}(x+2 \tau)^{3 / 2}} \varphi_{u}\right] g\left(\begin{array}{l}
\xi \\
\zeta
\end{array}\right) \frac{d \xi}{2 \tau \xi} d \zeta .
$$

On the other hand, since the function $\varphi$ is compactly supported in $B_{1}$, integration by parts implies that

$$
\int \varphi_{u}\left(M_{\tau}^{-1}\right) \frac{d \xi}{2 \tau \xi} d \zeta=\int \varphi_{u u}\left(M_{\tau}^{-1}\right) \frac{d \xi}{2 \tau \xi} d \zeta=0 .
$$

Hence we can express $x h_{x x}$ as

$$
x h_{x x}=I_{1}+I_{2},
$$

with

$$
I_{1}=\int_{0}^{t} d \tau \int \frac{x}{2 \tau(x+2 \tau)} \varphi_{u u}\left(M_{\tau}^{-1}\right)\left[g\left(\begin{array}{c}
\xi \\
\zeta
\end{array}\right)-g\left(\begin{array}{c}
x+2 \tau \\
y
\end{array}\right)\right] \frac{d \xi}{2 \tau \xi} d \zeta
$$

and

$$
I_{2}=\int_{0}^{t} d \tau \int \frac{x}{2 \sqrt{2 \tau}(x+2 \tau)^{3 / 2}} \varphi_{u}\left(M_{\tau}^{-1}\right)\left[g\left(\begin{array}{c}
\xi \\
\zeta
\end{array}\right)-g\left(\begin{array}{c}
x+2 \tau \\
y
\end{array}\right)\right] \frac{d \xi}{2 \tau \xi} d \zeta .
$$

But on the support of $\varphi_{u}\left(M_{\tau}^{-1}(P)\right)$ we have

$$
\left|g\left(\begin{array}{c}
\xi \\
\zeta
\end{array}\right)-g\left(\begin{array}{c}
x+2 \tau \\
y
\end{array}\right)\right| \leq\|g\|_{C_{s}^{\alpha}} s\left[\left(\begin{array}{c}
\xi \\
\zeta
\end{array}\right),\left(\begin{array}{c}
x+2 \tau \\
y
\end{array}\right)\right]^{\alpha} \leq C\|g\|_{C_{s}^{\alpha}} \tau^{\alpha / 2} .
$$


Thus, since $x /(x+2 \tau) \leq 1$, we can estimate

$$
\left|x h_{x x}\right| \leq C|| g\left\|_{C_{s}^{\alpha}} \int_{0}^{t} \frac{\tau^{\alpha / 2}}{\tau} d \tau \int\left(\left|\varphi_{u}\right|+\left|\varphi_{u u}\right|\right)\left(M_{\tau}^{-1}\right) \frac{d \xi}{2 \tau \xi} d \zeta \leq C t^{\alpha / 2}\right\| g \|_{C_{s}^{\alpha}(\mathcal{S})} .
$$

We continue by estimating the $\mathcal{C}_{s}^{\alpha}$ seminorm of $x h_{x x}$. Let $P=\left(\begin{array}{l}x \\ y \\ t\end{array}\right)$ and $P^{\prime}=\left(\begin{array}{l}x^{\prime} \\ y^{\prime} \\ t^{\prime}\end{array}\right)$ be any two points in $\mathcal{S}$. We will present the computation in the case that $y=y^{\prime}$ and $t=t^{\prime}$. All the other cases are similar. We write

$$
x h_{x x}(P)-x^{\prime} h_{x x}\left(P^{\prime}\right)=I+J,
$$

with

$$
I=\int_{0}^{t} \frac{d \tau}{2 \tau} \int\left[\frac{x}{x+2 \tau} \varphi_{u u}(\widetilde{P})-\frac{x^{\prime}}{x^{\prime}+2 \tau} \varphi_{u u}\left(\widetilde{P}^{\prime}\right)\right] g\left(\begin{array}{l}
\xi \\
\zeta
\end{array}\right) \frac{d \xi}{2 \tau \xi} d \zeta
$$

and

$$
J=\int_{0}^{t} \frac{d \tau}{2 \sqrt{2 \tau}} \int\left[\frac{x}{(x+2 \tau)^{3 / 2}} \varphi_{u}(\widetilde{P})-\frac{x^{\prime}}{\left(x^{\prime}+2 \tau\right)^{3 / 2}} \varphi_{u}\left(\widetilde{P}^{\prime}\right)\right] g\left(\begin{array}{l}
\xi \\
\zeta
\end{array}\right) \frac{d \xi}{2 \tau \xi} d \zeta
$$

where $\widetilde{P}=M_{\tau}^{-1}(P)$ and $\widetilde{P}^{\prime}=M_{\tau}^{-1}\left(P^{\prime}\right)$. We will estimate $I$, since $J$ can be estimated in a very similar manner. Set $\eta=s\left[P, P^{\prime}\right]^{2} / 4$ and split $I$ into the following four terms:

$$
\begin{gathered}
I_{1}=\int_{\eta}^{t} \frac{d \tau}{2 \tau} \int\left[\frac{x}{x+2 \tau}-\frac{x^{\prime}}{x^{\prime}+2 \tau}\right] \varphi_{u u}(\widetilde{P})\left[g\left(\begin{array}{l}
\xi \\
\zeta
\end{array}\right)-g\left(\begin{array}{c}
x+2 \tau \\
y
\end{array}\right)\right] \frac{d \xi}{2 \tau \xi} d \zeta, \\
I_{2}=\int_{\eta}^{t} \frac{d \tau}{2 \tau} \int \frac{x^{\prime}}{x^{\prime}+2 \tau}\left[\varphi_{u u}(\widetilde{P})-\varphi_{u u}\left(\widetilde{P}^{\prime}\right)\right]\left[g\left(\begin{array}{c}
\xi \\
\zeta
\end{array}\right)-g\left(\begin{array}{c}
x+2 \tau \\
y
\end{array}\right)\right] \frac{d \xi}{2 \tau \xi} d \zeta, \\
I_{3}=\int_{0}^{\eta} \frac{d \tau}{2 \tau} \int \frac{x}{x+2 \tau} \varphi_{u u}(\widetilde{P})\left[g\left(\begin{array}{c}
\xi \\
\zeta
\end{array}\right)-g\left(\begin{array}{c}
x+2 \tau \\
y
\end{array}\right)\right] \frac{d \xi}{2 \tau \xi} d \zeta
\end{gathered}
$$

and

$$
I_{4}=\int_{0}^{\eta} \frac{d \tau}{2 \tau} \int \frac{x^{\prime}}{x^{\prime}+2 \tau} \varphi_{u u}\left(\widetilde{P}^{\prime}\right)\left[g\left(\begin{array}{l}
\xi \\
\zeta
\end{array}\right)-g\left(\begin{array}{c}
x^{\prime}+2 \tau \\
y
\end{array}\right)\right] \frac{d \xi}{2 \tau \xi} d \zeta .
$$

Here we made use of identities (I.11.5). We claim that

$$
I_{i} \leq C\|g\|_{C_{s}^{\alpha}} s\left[P, P^{\prime}\right]^{\alpha}, \quad i=1, \ldots, 4 .
$$

We begin by estimating $I_{1}$. Since

$$
\frac{x}{x+2 \tau}-\frac{x^{\prime}}{x^{\prime}+2 \tau}=\int_{x^{\prime}}^{x} \frac{2 \tau}{(\bar{x}+2 \tau)^{2}} d \bar{x},
$$

and (I.11.6) holds on the support of $\varphi_{u u}(\widetilde{P})$, while

we have

$$
\int\left|\varphi_{u u}\right|\left(M_{\tau}^{-1}(P)\right) \frac{d \xi}{2 \tau \xi} d \zeta \leq C
$$

$$
\left|I_{1}\right| \leq C|| g \|_{C_{s}^{\alpha}}\left|\int_{x^{\prime}}^{x} d \bar{x} \int_{\eta}^{t} \frac{\tau^{\alpha / 2}}{(\bar{x}+2 \tau)^{2}} d \tau\right| .
$$

Substituting in the last integral $\tau=\bar{x} s$, we easily conclude that

$$
\left|I_{1}\right| \leq C\|g\|_{C_{s}^{\alpha}}\left|\int_{x^{\prime}}^{x} \bar{x}^{-1+\alpha / 2} d \bar{x}\right| \leq C\|g\|_{C_{s}^{\alpha}}\left|\sqrt{x}-\sqrt{x^{\prime}}\right|^{\alpha}
$$


from which the desired estimate follows since $s\left[P, P^{\prime}\right]=\left|\sqrt{2 x}-\sqrt{2 x^{\prime}}\right|$. The estimate of $I_{2}$ is similar. We write this time

$$
\varphi_{u u}(\widetilde{P})-\varphi_{u u}\left(\widetilde{P}^{\prime}\right)=\int_{x^{\prime}}^{x} \varphi_{u u u}\left(M_{\tau}^{-1}(\bar{P})\right) \frac{1}{\sqrt{2 \tau(\bar{x}+2 \tau)}} d \bar{x},
$$

with $\bar{P}=\left(\begin{array}{c}\bar{x} \\ y \\ t\end{array}\right)$, and instead of (I.11.6) use the estimate

$$
\left|g\left(\begin{array}{l}
\xi \\
\zeta
\end{array}\right)-g\left(\begin{array}{c}
x+2 \tau \\
y
\end{array}\right)\right| \leq\|g\|_{C_{s}^{\alpha}}\left(\tau^{\alpha / 2}+s\left[P, P^{\prime}\right]^{\alpha}\right) \leq C\|g\|_{C_{s}^{\alpha}} \tau^{\alpha / 2}
$$

holding on the union of the supports of $\varphi_{u u}(\widetilde{P})$ and $\varphi_{u u}\left(\widetilde{P}^{\prime}\right)$ and for $\tau \geq \eta=$ $s\left[P, P^{\prime}\right]^{2} / 4$. Since

$$
\int\left|\varphi_{\text {uиu }}\right|\left(M_{\tau}^{-1}(\bar{P})\right) \frac{d \xi}{2 \tau \xi} d \zeta \leq C
$$

we have

$$
\left|I_{2}\right| \leq\left. C|| g\right|_{C_{s}^{\alpha}}\left|\int_{x^{\prime}}^{x} d \bar{x} \int_{\eta}^{t} \frac{\bar{x} \tau^{(\alpha-1) / 2}}{(\bar{x}+2 \tau)^{3 / 2}} d \tau\right|
$$

which, under the substitution $\tau=\bar{x} s$ in the second integral, gives us the desired estimate. It remains to estimate $I_{3}$, since the estimate for $I_{4}$ follows by symmetry. Because once more (I.11.6) holds on the support of $\varphi_{u}(\widetilde{P})$ we conclude

$$
\left|I_{3}\right| \leq C\|g\|_{C_{s}^{\alpha}} \int_{0}^{\eta} \frac{\tau^{\alpha / 2}}{\tau} d \tau \leq C\|g\|_{C_{s}^{\alpha}} \eta^{\alpha / 2}
$$

which immediately implies the desired estimate, since $\eta=s\left[P, P^{\prime}\right]^{2} / 4$. The rest of the calculations are similar.

We can extend the previous result to Hölder spaces of higher order derivatives.

I.11.7. Theorem. Assume for some nonnegative integer $k$ and some number $\alpha$ in $0<\alpha<1$, that $g \in \mathcal{C}_{s}^{k, \alpha}(\mathcal{S})$ and $f^{0} \in \mathcal{C}_{s}^{k, 2+\alpha}\left(\mathcal{S}_{0}\right)$. Then, there exists a function $h \in \mathcal{C}_{s}^{k, 2+\alpha}(\mathcal{S})$ such that

$$
h\left(\begin{array}{l}
x \\
y \\
0
\end{array}\right)=f^{0}\left(\begin{array}{l}
x \\
y
\end{array}\right) \quad \text { and } \quad \frac{\partial h}{\partial t}\left(\begin{array}{l}
x \\
y \\
0
\end{array}\right)=g\left(\begin{array}{l}
x \\
y \\
0
\end{array}\right)
$$

and

$$
\|h\|_{\mathcal{C}_{s}^{k, 2+\alpha}(\mathcal{S})} \leq C\left(\left\|f^{0}\right\|_{\mathcal{C}_{s}^{k, 2+\alpha}\left(\mathcal{S}_{0}\right)}+\|g\|_{\mathcal{C}_{s}^{k, \alpha}(\mathcal{S})}\right)
$$

for some constant $C$ depending only on $\alpha$ and $k$.

Proof. As before, the result can be easily reduced to the case that $f^{0} \equiv 0$. It can be shown now, via similar computations as in the proof of Theorem I.11.4, that if $g \in \mathcal{C}_{s}^{k, \alpha}(\mathcal{S})$, the function $h$ defined, as before, by

$$
h\left(\begin{array}{l}
x \\
y \\
t
\end{array}\right)=\int_{0}^{t} d \tau \int \varphi\left(\begin{array}{l}
u \\
v
\end{array}\right) g\left(M_{\tau}\left(\left(\begin{array}{l}
x \\
y
\end{array}\right) ;\left(\begin{array}{l}
u \\
v
\end{array}\right)\right)\right) d u d v
$$

belongs in the space $\mathcal{C}_{s}^{k, 2+\alpha}(\mathcal{S})$ and satisfies

$$
\|h\|_{\mathcal{C}_{s}^{k, 2+\alpha}(\mathcal{S})} \leq C\|g\|_{\mathcal{C}_{s}^{k, \alpha}(\mathcal{S})} .
$$


Before we finish this section we will introduce smoothing operators in space and time. Let $\widetilde{P}=\left(\begin{array}{l}x \\ y \\ t\end{array}\right)$ be a point in $\mathcal{S}=\mathcal{S}_{0} \times[0, \infty)$ and $\widetilde{Q}=\left(\begin{array}{l}u \\ v \\ s\end{array}\right)$ any point in the unit box $\widetilde{\mathcal{B}_{1}}=\{|u|<1,|v| \leq 1,|s| \leq 1\}$. For $\epsilon>0$ let $M_{\epsilon}$ denote the spatial regularizations introduced in the beginning of this section. Starting from the point $\widetilde{P}$, we define now the new point

$$
\widetilde{M}_{\epsilon}(\widetilde{P} ; \widetilde{Q})=\left(\begin{array}{l}
\xi \\
\zeta \\
\tau
\end{array}\right),
$$

having

$$
\left(\begin{array}{l}
\xi \\
\zeta
\end{array}\right)=M_{\epsilon}\left(\left(\begin{array}{l}
x \\
y
\end{array}\right) ;\left(\begin{array}{l}
u \\
v
\end{array}\right)\right)
$$

and

$$
\tau=t+2 \epsilon+\epsilon s
$$

The following Lemma is a direct consequence of the above definitions and Lemma I.11.2.

I.11.8. Lemma. For any two points $\widetilde{P}, \widetilde{P}^{\prime}$ in $\mathcal{S}$ and any point $\widetilde{Q}$ in the box $\widetilde{B}_{1}$, we have

$$
s\left[\widetilde{M}_{\epsilon}(\widetilde{P} ; \widetilde{Q}), \widetilde{M}_{\epsilon}\left(\widetilde{P}^{\prime} ; \widetilde{Q}\right)\right] \leq C s\left[\widetilde{P}, \widetilde{P}^{\prime}\right]
$$

and

$$
s\left[\widetilde{M}_{\epsilon}(\widetilde{P} ; \widetilde{Q}), \widetilde{P}\right] \leq C \sqrt{\epsilon} .
$$

Now let $g$ be a continuous function on $\mathcal{S}$ and let $\widetilde{\varphi}$ be a standard smooth, nonnegative bumb function, supported in $\widetilde{\mathcal{B}_{1}}$, and such that

$$
\int d s \int \widetilde{\varphi}\left(\begin{array}{l}
u \\
v \\
s
\end{array}\right) d u d v=1
$$

We define the regularizations $g_{\epsilon}$ of $g$ as

$$
g_{\epsilon}(\widetilde{P})=\int d s \int \widetilde{\varphi}(\widetilde{Q}) g\left(\widetilde{M}_{\epsilon}(\widetilde{P} ; \widetilde{Q})\right) d u d v=\int \frac{d \tau}{\epsilon} \int \varphi\left(\widetilde{M}_{\epsilon}^{-1}(\widetilde{P} ; \widetilde{R})\right) g(\widetilde{R}) \frac{d \xi}{2 \epsilon \xi} d \zeta
$$

where $\widetilde{R}=M_{\epsilon}(\widetilde{P} ; \widetilde{Q})$. As an immediate consequence of Theorem I.11.3, we obtain the following space-time regularizing result:

I.11.9. Theorem. For any function $g$ in $\mathcal{C}_{s}^{\alpha}(\mathcal{S})$ and any two points $\widetilde{P}$ and $\widetilde{P}^{\prime}$ in $\mathcal{S}$, we have

$$
\left\|g_{\epsilon}\right\|_{\mathcal{C}_{s}^{\alpha}(\mathcal{S})} \leq C\|g\|_{\mathcal{C}_{s}^{\alpha}(\mathcal{S})}
$$

and

$$
\left|g_{\epsilon}(\widetilde{P})-g\left(\widetilde{P}^{\prime}\right)\right| \leq C \epsilon^{\alpha / 2}\|g\|_{\mathcal{C}_{s}^{\alpha}(\mathcal{S})}
$$

with $C$ independent of $\epsilon$. 
I.12. Existence and uniqueness. In this last section of Part I we will give the proof of the existence and uniqueness Theorem I.1.1.

Proof of Theorem I.1.1. We begin with the existence question. Because of Theorem I.11.7, we can assume without loss of generality that $f^{0} \equiv 0$ and that $g$ is a function in $\mathcal{C}_{s}^{k, \alpha}(\mathcal{S})$ such that

$$
g\left(\begin{array}{l}
x \\
y \\
0
\end{array}\right)=0 \quad \forall\left(\begin{array}{l}
x \\
y
\end{array}\right) \in \mathcal{S}_{0} .
$$

Let $g_{\epsilon}$ be the space-time regularizations of the function $g$, as defined at the end of the previous section. Each $g_{\epsilon}$ is smooth, compactly supported in $\mathcal{S}=\mathcal{S}_{0} \times[0, \infty)$ and vanishes at $t=0$. In addition, it follows from Theorem I.11.9, that

$$
\left\|g_{\epsilon}\right\|_{\mathcal{C}_{s}^{k, \alpha}(\mathcal{S})} \leq C\|g\|_{\mathcal{C}_{s}^{k, \alpha}(\mathcal{S})}
$$

and

$$
g_{\epsilon} \rightarrow g, \quad \text { as } \quad \epsilon \rightarrow 0
$$

uniformly on $\mathcal{S}$. Let $f_{\epsilon}$ be the unique solution of the initial value problem

$$
\begin{cases}L_{0} f_{\epsilon}=g_{\epsilon} & \text { in } \mathcal{S}, \\ f_{\epsilon}(\cdot, 0)=0 & \text { on } \mathcal{S}_{0}\end{cases}
$$

satisfying

$$
\left\|f_{\epsilon}\right\|_{\mathcal{C}^{0}(\mathcal{S})} \leq C\left\|g_{\epsilon}\right\|_{\mathcal{C}^{0}(\mathcal{S})}
$$

as constructed in Theorem I.1.2. The Schauder estimate in Theorem I.1.3 implies that for every compact subset $\mathcal{K}$ of $\mathcal{S}$ we have

$$
\left\|f_{\epsilon}\right\|_{\mathcal{C}_{s}^{k, 2+\alpha}(\mathcal{K})} \leq C\left\|g_{\epsilon}\right\|_{\mathcal{C}_{s}^{k, \alpha}(\mathcal{S})}
$$

with $C$ independent of $\mathcal{K}$. Let $0<\beta<\alpha$. It follows from the previous estimate that there exists a subsequence $\left\{f_{\epsilon_{n}}\right\}$ of $\left\{f_{\epsilon}\right\}$ which converges in $\mathcal{C}^{k, 2+\beta}(\mathcal{K})$ to a function $f$, for all compact subsets $\mathcal{K}$ of $\mathcal{S}$. It is now easy to conclude that $f$ belongs to the space $\mathcal{C}^{k, 2+\alpha}(\mathcal{S})$ and satisfies

$$
\|f\|_{\mathcal{C}^{k, 2+\alpha}(\mathcal{S})} \leq C\|g\|_{\mathcal{C}_{s}^{k, \alpha}(\mathcal{S})}
$$

as desired.

The uniqueness of solutions follows from the classical maximum principle, as in Theorem I.3.1. After a moment of thought we come to the conclusion that the maximum of a solution $f$ of the equation

$$
f_{t}-x\left(f_{x x}+f_{y y}\right)-\nu f_{x}=0
$$

cannot occur at the boundary $x=0$ since

$$
x f_{x x}=x f_{y y}=0, \quad \text { at } x=0
$$

for all functions $f \in C_{s}^{k, 2+\alpha}(\mathcal{S})$ and $\nu>0$. The last identities obviously hold true when $k \geq 1$, in which case every function $f$ in $C_{s}^{k, 2+\alpha}(\mathcal{S})$ is at least twice differentiable up to the boundary $x=0$. When $f \in C_{s}^{2+\alpha}(\mathcal{S})$ we still have $x f_{x x}=$ $x f_{y y}=0$ at $x=0$, as shown next.

I.12.1. Proposition. Every function $f \in C_{s}^{2+\alpha}(\mathcal{S})$ satisfies

$$
x f_{x x}=x f_{y y}=0, \quad \text { at } x=0 .
$$


Proof. We will show that $x f_{x x}=0$ at $x=0$, as the rest of the identities follow via similar arguments. We will proceed by contradiction. Assume that for some point $-\infty<y_{0}<\infty$ we have

$$
\lim _{x \rightarrow 0} x f_{x x}\left(x, y_{0}\right)=a \neq 0
$$

and assume that $a>0$, otherwise replace $f$ by $-f$. Since the function $x f_{x x}$ is continuous up to the boundary $x=0$, there exists a number $\delta>0$ such that

$$
x f_{x x}(x, y) \geq \frac{a}{2}
$$

for all $0 \leq x \leq \delta, y_{0}-\delta \leq y \leq y_{0}+\delta$. But then

$$
f_{x}\left(\delta, y_{0}\right)-f_{x}\left(0, y_{0}\right)=\int_{0}^{\delta} f_{x x}\left(x, y_{0}\right) d x \geq \int_{0}^{\delta} \frac{a}{2 x} d x=\infty
$$

is impossible, since $f_{x}$ is continuous up to the boundary $x=0$. Therefore, $x f_{x x}=0$ at $x=0$, finishing the proof of the proposition.

We conclude this section with the following generalization of Theorem I.1.1 which will be used in Part II of the paper. For a number $T>0$ we denote, as before, by $\mathcal{S}_{T}$ the space $\mathcal{S}_{0} \times[0, T]$.

I.12.2. Theorem. Let $k$ be a nonnegative integer and $\alpha$ a number in $0<\alpha<1$. Assume that $g \in \mathcal{C}_{s}^{k, \alpha}(\mathcal{S})$ and $f^{0} \in \mathcal{C}_{s}^{k, 2+\alpha}\left(\mathcal{S}_{0}\right)$, with both $g$ and $f^{0}$ compactly supported in $\mathcal{S}$ and $\mathcal{S}_{0}$ respectively. Then, for any constant $c$ and any $\nu>0$ and $T>0$, the initial value problem

$$
\begin{cases}L_{0} f-c f=g & \text { in } \mathcal{S}_{T}, \\ f(\cdot, 0)=f^{0} & \text { on } S_{0}\end{cases}
$$

admits a unique solution $f \in \mathcal{C}_{s}^{k, 2+\alpha}\left(\mathcal{S}_{T}\right)$ which satisfies the estimate

$$
\|f\|_{\mathcal{C}_{s}^{k, 2+\alpha}\left(\mathcal{S}_{T}\right)} \leq C(T)\left(\left\|f^{0}\right\|_{\mathcal{C}_{s}^{k, 2+\alpha}\left(\mathcal{S}_{0}\right)}+\|g\|_{\mathcal{C}_{s}^{k, \alpha}\left(\mathcal{S}_{T}\right)}\right)
$$

for some constant $C(T)$ depending on $k, \alpha, \nu, c$ and $T$.

Proof. The uniqueness assertion of the Theorem follows, as in Theorem I.3.1, from a direct consequence of the classical maximum principle.

For existence, you just need to observe that $f$ is the desired solution if and only if $\tilde{f}=e^{-c t} f$ is a solution to the problem

$$
\begin{cases}L_{0} \tilde{f}=e^{-c t} g & \text { in } \mathcal{S}_{T}, \\ f(\cdot, 0)=f^{0} & \text { on } S_{0}\end{cases}
$$

which can be solved by Theorem I.1.1. It is clear that the constant $C(T)$ will also depend on $c$.

\section{Part II. Degenerate equations With Variable CoefFicients}

II.1. The linear case. In this section we will study linear degenerate equations of the form

$$
w_{t}=\left(\vartheta a^{i j} w_{i j}+b^{i} w_{i}+c w\right)+g
$$

on the cylinder $\Omega \times[0, \infty)$, where $\Omega$ is a compact domain in $\mathcal{R}^{2}$ with smooth boundary. The subindices $i, j \in\{x, y\}$ denote differentiation with respect to the 
space variables $x, y$ and the summation convention is used. We assume that the coefficient matrix $\left(a^{i j}\right)$ is strictly positive and all coefficients $a^{i j}, b^{i}$ and $c$ belong to appropriate Hölder spaces which will be defined later.

The degeneracy of the equation is carried through the function $\vartheta(x)$ which is assumed to be smooth on $\Omega$, strictly positive in its interior, with

$$
\vartheta(P)=\operatorname{dist}(P, \partial \Omega)
$$

for all $P \in \Omega$ sufficiently close to $\partial \Omega$. We also assume that

$$
b^{i} n_{i}>0 \quad \text { on } \partial \Omega
$$

where $n=\left(n_{1}, n_{2}\right)$ denotes the interior normal to the boundary of $\Omega$ and again the summation convention is used.

When the boundary is flat and the coefficients are constants, this equation (locally and under an appropriate change of variables) takes the form of the model equation studied in Part I

$$
f_{t}=x\left(f_{x x}+f_{y y}\right)+\nu f_{x}+g
$$

on the half-space $x \geq 0$. The condition $b^{i} n_{i}>0$ at $\partial \Omega$ is then equivalent to the condition $\nu>0$. Notice that, as in the constant coefficient model, we don't impose any conditions on $w$ along the boundary of $\Omega$, where the principal coefficients $\vartheta a^{i j}$ vanish. Because of the condition $b^{i} n_{i}>0$ at $\partial \Omega$, the lateral boundary of the cylinder $\Omega \times[0, \infty)$ behaves like a free boundary.

Imitating the model case where the operators are defined on the half-space $\{x \geq$ $0\}$, we define the cycloidal distance function $s$ in $\Omega$. In the interior of $\Omega$ the cycloidal distance will be equivalent to the standard Euclidean distance, while around any point $P \in \partial \Omega, s$ is defined as the pull back of the cycloidal distance on the halfspace $\mathcal{S}=\{x \geq 0\}$, as defined in Part I, via a map $\varphi: \mathcal{S} \rightarrow \Omega$ that straightens the boundary of $\Omega$ near $P$.

It can be easily shown that the cycloidal distance between two points $P_{1}=\left(\begin{array}{l}x_{1} \\ y_{1}\end{array}\right)$ and $P_{2}=\left(\begin{array}{l}x_{2} \\ y_{2}\end{array}\right)$ in $\Omega$ is equivalent to the function

$$
\bar{s}\left(P_{1}, P_{2}\right)=\frac{\left|P_{1}-P_{2}\right|}{\sqrt{\left|P_{1}-P_{2}\right|}+\sqrt{d\left(P_{1}\right)}+\sqrt{d\left(P_{2}\right)}}
$$

with $d=d(P)$ denoting the distance to the boundary of $\Omega$.

The parabolic distance in the cycloidal metric is equivalent to the function

$$
\bar{s}\left[\left(\begin{array}{c}
P_{1} \\
t_{1}
\end{array}\right),\left(\begin{array}{c}
P_{2} \\
t_{2}
\end{array}\right)\right]=s\left(P_{1}, P_{2}\right)+\sqrt{\left|t_{1}-t_{2}\right|} .
$$

Now suppose that $\mathcal{A}$ is a subset of the cylinder $\Omega \times[0, \infty)$ which is the closure of its interior. As in Part I, we denote by $\mathcal{C}_{s}^{\alpha}(\mathcal{A})$ the space of Hölder continuous functions on $\mathcal{A}$ with respect to the metric $s$ and by $\mathcal{C}_{s}^{2+\alpha}(\mathcal{A})$ the space of all functions $w$ on $\mathcal{A}$ such that $w, w_{t}, w_{i}$ and $d w_{i j}$, with $i, j \in\{x, y\}$ and with $d$ denoting the distance function to the boundary of $\Omega$, extend continuously up to the boundary of $\mathcal{A}$ and the extensions are Hölder continuous on $\mathcal{A}$ of class $C_{s}^{\alpha}(\mathcal{A})$. They are both Banach spaces under the norms $\|w\|_{\mathcal{C}_{s}^{\alpha}(\mathcal{A})}$, as defined in Part I, and

$$
\|w\|_{\mathcal{C}_{s}^{2+\alpha}(\mathcal{A})}=\|w\|_{\mathcal{C}_{s}^{\alpha}(\mathcal{A})}+\left\|w_{i}\right\|_{\mathcal{C}_{s}^{\alpha}(\mathcal{A})}+\left\|d w_{i j}\right\|_{\mathcal{C}_{s}^{\alpha}(\mathcal{A})}
$$

respectively, where once more the summation convention is used. Also, in analogy to the corresponding definitions in Part I, we denote by $C_{s}^{k, \alpha}(\mathcal{A})$ and $C_{s}^{k, 2+\alpha}(\mathcal{A})$ the spaces of all functions $w$ whose $k$-th order derivatives $D_{x}^{i} D_{y}^{j} D_{t}^{l} w$ with $i+j+l=k$ 
exist and belong to the spaces $C_{s}^{\alpha}(\mathcal{A})$ and $C_{s}^{2+\alpha}(\mathcal{A})$ respectively. Both spaces equipped with the norms

$$
\|w\|_{C_{s}^{k, \alpha}(\mathcal{A})}=\sum_{i+j+l \leq k}\left\|D_{x}^{i} D_{y}^{j} D_{t}^{l} w\right\|_{C_{s}^{\alpha}(\mathcal{A})}
$$

and

$$
\|w\|_{C_{s}^{k, 2+\alpha}(\mathcal{A})}=\sum_{i+j+l \leq k}\left\|D_{x}^{i} D_{y}^{j} D_{t}^{l} w\right\|_{C_{s}^{2+\alpha}(\mathcal{A})}
$$

respectively, are Banach spaces. We will denote by $C_{s}^{\alpha}(\mathcal{A})$ and $C_{s}^{2+\alpha}(\mathcal{A})$ the spaces $C_{s}^{0, \alpha}(\mathcal{A})$ and $C_{s}^{0,2+\alpha}(\mathcal{A})$ respectively.

Denoting by $L$ the operator

$$
L w=w_{t}-\left(\vartheta a^{i j} w_{i j}+b^{i} w_{i}+c w\right)
$$

by $\Omega_{\sigma}$, for $\sigma>0$, the set

$$
\Omega_{\sigma}=\{x \in \Omega: \operatorname{dist}(x, \partial \Omega) \geq \sigma\},
$$

by $Q_{T}$, for $T>0$, the cylinder $\Omega \times[0, T]$ and, as always, by $n=\left(n_{1}, n_{2}\right)$ the interior unit normal to $\partial \Omega$, we can now state the main result in Section II.1:

II.1.1. Theorem (Existence and Uniqueness). Let $\Omega$ be a compact domain in $\mathcal{R}^{2}$ with smooth boundary and let $k$ be a nonnegative integer, a a number in $0<a<1$ and $T$ a positive number. Assume that the coefficients $a^{i j}, b^{i}$ and $c$ of the operator $L$ belong to the space $C_{s}^{k, \alpha}\left(Q_{T}\right)$ and satisfy the ellipticity condition

$$
a^{i j} \xi_{i} \xi_{j} \geq \lambda|\xi|^{2}>0 \quad \forall \xi \in \mathcal{R}^{2} \backslash\{0\}
$$

and the bounds

$$
\left\|a^{i j}\right\|_{C_{s}^{k, \alpha}\left(Q_{T}\right)} \quad\left\|b^{i}\right\|_{C_{s}^{k, \alpha}\left(Q_{T}\right)} \quad\|c\|_{C_{s}^{k, \alpha}\left(Q_{T}\right)} \leq 1 / \lambda
$$

and

$$
b^{i} n_{i} \geq \nu>0 \quad \text { on } \quad \partial \Omega \times[0, T]
$$

for some positive constants $\lambda$ and $\nu$. In addition, assume that $\vartheta$ is a smooth function on $\Omega$, strictly positive in its interior, with $\|\vartheta\|_{C^{\infty}(\Omega)} \leq 1$ and such that

$$
\vartheta(P)=\operatorname{dist}(P, \partial \Omega) \quad \forall P \in \Omega \backslash \Omega_{\sigma}
$$

for some $\sigma>0$. Then, given any funtion $w^{0} \in C_{s}^{k, 2+\alpha}(\Omega)$ and any function $g \in C_{s}^{k, \alpha}\left(Q_{T}\right)$ there exists a unique solution $w \in C_{s}^{k, 2+\alpha}\left(Q_{T}\right)$ of the initial value problem

satisfying

$$
\left\{\begin{array}{lll}
L w=g & \text { in } & Q_{T} \\
w(\cdot, 0)=w^{0} & \text { on } & \Omega
\end{array}\right.
$$

$$
\|w\|_{\mathcal{C}_{s}^{k, 2+\alpha}\left(Q_{T}\right)} \leq C(T)\left(\left\|w^{0}\right\|_{\mathcal{C}_{s}^{k, 2+\alpha}(\Omega)}+\|g\|_{\mathcal{C}_{s}^{k, \alpha}\left(Q_{T}\right)}\right) .
$$

The constant $C(T)$ depends only on the domain $\Omega$ and the numbers $\alpha, k, \lambda, \nu, \sigma$ and $T$.

Proof. Because of the extension Theorem I.11.7 we can assume, without loss of generality, that $f \equiv 0$ and that $g$ is a function in $C_{s}^{k, \alpha}\left(Q_{T}\right)$, which vanishes at $t=0$. 
For $\delta>0$, set $Q_{\delta}=\Omega \times[0, \delta]$ and denote by $C_{s, 0}^{k, 2+\alpha}\left(Q_{\delta}\right)$ and $C_{s, 0}^{k, \alpha}\left(Q_{\delta}\right)$ the subspaces of $C_{s}^{k, 2+\alpha}\left(Q_{\delta}\right)$ and $C_{s}^{k, \alpha}\left(Q_{\delta}\right)$ respectively, consisting of all functions which vanish identically at $t=0$. Also, denote by $I$ the identity operator on $C_{s, 0}^{k, \alpha}\left(Q_{\delta}\right)$. We will show that, if $\delta$ is sufficiently small, there exists an operator $M: C_{s, 0}^{k, \alpha}\left(Q_{\delta}\right) \rightarrow$ $C_{s, 0}^{k, 2+\alpha}\left(Q_{\delta}\right)$ such that

$$
\|L M-I\| \leq \frac{1}{2}
$$

This will immediately imply that the operator $L M: C_{s, 0}^{k, \alpha}\left(Q_{\delta}\right) \rightarrow C_{s, 0}^{k, \alpha}\left(Q_{\delta}\right)$ is invertible and therefore $L: C_{s, 0}^{k, 2+\alpha}\left(Q_{\delta}\right) \rightarrow C_{s, 0}^{k, \alpha}\left(Q_{\delta}\right)$ will be onto, as desired.

We begin by expressing the compact domain $\Omega$ as the finite union

$$
\Omega=\Omega_{0} \cup\left(\bigcup_{l \geq 1} \Omega_{l}\right)
$$

of compact domains in such a way that

$$
\operatorname{dist}\left(\Omega_{0}, \partial \Omega\right) \geq \frac{\rho}{2}>0
$$

and for all $l \geq 1$

$$
\Omega_{l}=B_{\rho}\left(x_{l}\right) \cap \Omega
$$

with $B_{\rho}\left(x_{l}\right)$ denoting the ball centered at $x_{l} \in \partial \Omega$ of radius $\rho>0$. The number $\rho>0$ will be determined later.

The operator $L$, when restricted on the interior domain $\Omega_{0}$ is nondegenerate. Therefore, the classical Schauder theory for linear parabolic equations implies that $L$ is invertible when restricted on functions which vanish outside $\Omega_{0}$. Notice that our Hölder spaces with respect to the cycloidal metric $s$ on the interior domain $\Omega_{0}$ coincide with the standard Hölder spaces, where the classical Schauder theory holds true. Denote by $M_{0}: C_{s, 0}^{k, \alpha}\left(\Omega_{0} \times[0, \delta]\right) \rightarrow C_{s, 0}^{k, 2+\alpha}\left(\Omega_{0} \times[0, \delta]\right)$ the inverse of the operator $L$ restricted on $\Omega_{0}$.

Next, we concentrate our attention on the domains $\Omega_{l}, l \geq 1$, close to the boundary of $\Omega$, which can be chosen in such a way that the sets $B_{\rho / 4}\left(x_{l}\right) \cap \Omega$ are disjoint. Denoting by $\bar{B}$ the half unit ball

$$
\bar{B}=\left\{(x, y) \in B_{1}(0) ; x \geq 0\right\}
$$

and by $\bar{Q}_{\delta}$ the cylinder

$$
\bar{Q}_{\delta}=\bar{B} \times[0, \delta]
$$

we select smooth charts $\Upsilon_{l}: \bar{B} \rightarrow \Omega_{l}$, which flatten the boundary of $\Omega$, i.e., they map $\bar{B} \cap\{x=0\}$ onto $\Omega_{l} \cap \partial \Omega$ and have $\Upsilon_{l}(0)=x_{l}$. This is possible if the number $\rho$ is chosen sufficiently small. Under the change of coordinates induced by the charts $\Upsilon_{l}$, the operator $L$, restricted on each $\Omega_{l} \times[0, \delta]$, is transformed to an operator $\bar{L}_{l}$ of the form

$$
\bar{L}_{l} \bar{w}=\bar{w}_{t}-\left(x \bar{a}_{l}^{i j} \bar{w}_{i j}+\bar{b}_{l}^{i} \bar{w}_{i}+\bar{c}_{l} \bar{w}\right)
$$

defined on $\bar{B} \times[0, \delta]$. Moreover, the charts $\Upsilon_{l}$ can be chosen appropriately so that the coefficients of $\bar{L}_{l}$ satisfy

$$
\bar{a}_{l}^{i j} \xi_{i} \xi_{j} \geq \lambda|\xi|^{2}>0 \quad \forall \xi \in \mathcal{R}^{2} \backslash\{0\}
$$


and

$$
\left\|\bar{a}_{l}^{i j}\right\|_{C_{s}^{k, \alpha}\left(\bar{Q}_{\delta}\right)}, \quad\left\|\bar{b}_{l}^{i}\right\|_{C_{s}^{k, \alpha}\left(\bar{Q}_{\delta}\right)}, \quad\left\|\bar{c}_{l}\right\|_{C_{s}^{k, \alpha}\left(\bar{Q}_{\delta}\right)} \leq 1 / \bar{\lambda}
$$

and

$$
\bar{b}_{l}^{i} \geq \bar{\nu}>0, \quad \text { at } x=0
$$

for some positive constants $\bar{\lambda}$ and $\bar{\nu}$, while at the point $(0,0)$ we have

$$
\bar{a}_{l}^{11}(0,0)=\bar{a}_{l}^{22}(0,0), \quad \bar{a}_{l}^{i j}(0,0)=0, \quad \text { for } i \neq j
$$

and

$$
\bar{b}_{l}^{1}(0,0) \geq \bar{\nu}>0, \quad \bar{b}_{l}^{2}(0,0)=0 .
$$

Here the index $i=1$ corresponds to the variable $x$ and $j=2$ to the variable $y$. The continuity of the coefficients then implies that the constant coefficient operator

$$
\widetilde{L}_{l} \bar{w}=\bar{w}_{t}-\left[x \bar{a}_{l}\left(\bar{w}_{x x}+\bar{w}_{y y}\right)+\bar{\nu}_{l} \bar{w}_{x}+\bar{c}_{l} \bar{w}\right]
$$

having

$$
\bar{a}_{l}=a_{l}^{11}(0,0)=a_{l}^{22}(0,0), \quad \bar{\nu}_{l}=\bar{b}_{l}^{1}(0,0), \quad \bar{c}_{l}=\bar{c}_{l}(0,0)
$$

when defined on $\bar{Q}_{\delta}=\bar{B} \times[0, \delta]$ has coefficients sufficiently close to the coefficients of $\bar{L}_{l}$ in the space $C_{s}^{k, \alpha}\left(Q_{\delta}\right)$, if $\rho$ and $\delta$ are chosen sufficiently small, depending only on $\Omega$ and the constants $\sigma$ and $\lambda$ in the statement of the theorem. Notice that each of the operators $\widetilde{L}_{l}$ has the form of the model operators studied in Part I.

Denote, as in Part I, by $\mathcal{S}_{0}$ the half-space $x \geq 0$ in $\mathcal{R}^{2}$ and by $\mathcal{S}_{\delta}$ the space $\mathcal{S}_{0} \times[0, \delta]$. Also, consider the subspace $\bar{C}_{s, 0}^{k, \alpha}\left(\mathcal{S}_{\delta}\right)$ of $C_{s, 0}^{k, \alpha}\left(\mathcal{S}_{\delta}\right)$, consisting of functions which are compactly supported on $\mathcal{S}_{\delta}$. Then, Theorem I.12.2 implies that for every $l=1,2, \ldots$ there is an operator $\widetilde{M}_{l}: \bar{C}_{s, 0}^{k, \alpha}\left(\mathcal{S}_{\delta}\right) \rightarrow C_{s, 0}^{k, 2+\alpha}\left(\mathcal{S}_{\delta}\right)$ such that

$$
\widetilde{L}_{l} \widetilde{M}_{l}=I
$$

with $I$ denoting the identity operator on $\bar{C}_{s, 0}^{k, \alpha}\left(\mathcal{S}_{\delta}\right)$. Denote by $M_{l}$ the pull back of the operator $\widetilde{M}_{l}$ via the chart $\Upsilon_{l}$. Next, choose a nonnegative partition of unity $\phi_{l}, l=0,1, \ldots$, subordinated to the cover $\Omega_{l}, l=0,1, \ldots$, of $\Omega$ and also choose, for each $l \geq 0$, nonnegative, smooth bump functions $\psi_{l}, 0 \leq \psi_{l} \leq 1$, supported in $\Omega_{l}$ with $\psi_{l} \equiv 1$ on the support of $\phi_{l}$. Then $\sum_{l \geq 0} \phi_{l}=1$ and $\psi_{l} \phi_{l}=\phi_{l}$ for all $l$.

Our goal is to show that the operator $M: C_{s, 0}^{k, \alpha}\left(Q_{\delta}\right) \rightarrow C_{s, 0}^{k, 2+\alpha}\left(Q_{\delta}\right)$ defined as

$$
M g=\sum \psi_{l} M_{l} \phi_{l} g
$$

satisfies

$$
\|L M g-g\|_{C_{s}^{\alpha}\left(Q_{\delta}\right)}<\frac{1}{2}\|g\|_{C_{s}^{\alpha}\left(Q_{\delta}\right)} \quad \forall g \in C_{s, 0}^{k, \alpha}\left(Q_{\delta}\right)
$$

if the cover $\left\{\Omega_{l}\right\}$ and $\delta$ are chosen appropriately. Indeed, we can write

$$
L M g-g=\sum_{l} L \psi_{l} M_{l} \phi_{l} g-\sum_{l} \phi_{l} g=\sum_{l} \psi_{l}\left(L M_{l}-I\right) \phi_{l} g+\sum_{l}\left[L, \psi_{l}\right] M_{l} \phi_{l} g
$$


with $\left[L, \psi_{l}\right]$ denoting the commutator of $L$ and $\psi_{l}$. The commutator $\left[L, \psi_{l}\right]$ is only of first order and it can be estimated as

$$
\left\|\left[L, \psi_{l}\right] M_{l} \phi_{l} g\right\|_{C_{s}^{k, \alpha}\left(Q_{\delta}\right)} \leq C\left(\left\|\vartheta D\left(M_{l} \phi_{l} g\right)\right\|_{C_{s}^{k, \alpha}\left(Q_{\delta}\right)}+\left\|M_{l} \phi_{l} g\right\|_{C_{s}^{k, \alpha}\left(Q_{\delta}\right)}\right) .
$$

Let $\epsilon>0$. Since the function $\vartheta$ is proportional to the distance $d$ to the boundary of $\Omega$, it follows by the standard interpolation between Hölder spaces that

$$
\left\|\vartheta D\left(M_{l} \phi_{l} g\right)\right\|_{C_{s}^{k, \alpha}\left(Q_{\delta}\right)} \leq \epsilon\left\|M_{l} \phi_{l} g\right\|_{C_{s}^{k, 2+\alpha}\left(Q_{\delta}\right)}+C(\epsilon)\left\|M_{l} \phi_{l} g\right\|_{C^{k, 0}\left(Q_{\delta}\right)} .
$$

However, for each $k$ we have

$$
\left\|M_{l} \phi_{l} g\right\|_{C_{s}^{k, 2+\alpha}\left(Q_{\delta}\right)} \leq C\|g\|_{C_{s}^{k, \alpha}\left(Q_{\delta}\right)}
$$

and therefore, since $M_{l} \phi_{l} g \equiv 0$ at $t=0$,

$$
\left\|M_{l} \phi_{l} g\right\|_{C_{s}^{k, 0}\left(Q_{\delta}\right)} \leq C \delta\|g\|_{C_{s}^{k, \alpha}\left(Q_{\delta}\right)} .
$$

Therefore if we choose $\delta$ sufficiently small we can make

$$
\sum_{l}\left\|\left[L, \psi_{l}\right] M_{l} \phi_{l} g\right\|_{C_{s}^{k, \alpha}\left(Q_{\delta}\right)} \leq \frac{1}{4}\|g\|_{C_{s}^{k, \alpha}\left(Q_{\delta}\right)} .
$$

On the other hand we have $\left(L M_{0}-I\right) \varphi_{0} g=0$, while for $l \geq 1$, we can make the norm of each of the operators $L M_{l}-I$ arbitrarily close to zero by choosing the diameters of the domains $\Omega_{l}$ sufficiently small. Wherein

$$
\left\|\sum_{l} \psi_{l}\left(L M_{l}-I\right) \phi_{l} g\right\|_{C_{s}^{k, \alpha}\left(Q_{\delta}\right)}<\frac{1}{4}\|g\|_{C_{s}^{k, \alpha}\left(Q_{\delta}\right)}
$$

for all $g \in C_{s}^{k, \alpha}\left(Q_{\delta}\right)$, if $\rho$ and $\delta$ are both sufficiently small. Combining the above estimates we obtain that

$$
\|L M g-g\|_{C_{s}^{k, \alpha}\left(Q_{\delta}\right)} \leq \frac{1}{2}\|g\|_{C_{s}^{k, \alpha}\left(Q_{\delta}\right)}
$$

for all $g \in C_{s, 0}^{k, \alpha}\left(Q_{\delta}\right)$, as desired. We conclude that for every $g \in C_{s, 0}^{k, \alpha}\left(Q_{\delta}\right)$ there exists a function $w \in C_{s, 0}^{k, 2+\alpha}\left(Q_{\delta}\right)$ such that $L w=g$. In addition

$$
\|w\|_{C_{s}^{k, 2+\alpha}\left(Q_{\delta}\right)} \leq C\|g\|_{C^{k, \alpha}\left(Q_{\delta}\right)}
$$

with $C$ depending only on $\Omega$ and the constants $\nu, \lambda$ and $\sigma$. This proves short time existence. The long time existence follows immediately by the last estimate.

The uniqueness of solutions follows by applying the classical maximum principle as in Theorem I.3.1.

We give next the generalization of the local Schauder estimates in Theorem I.1.3 for variable coefficient equations. For simplicity we will assume that the operator $L$ has the form

$$
L w=w_{t}-\left(x a^{i j} w_{i j}+b^{i} w_{i}+c w\right)
$$

defined on the half-space $x \geq 0$. As at the beginning of Part I, we define the box of side $r$ around a point $P=\left(\begin{array}{l}x_{0} \\ y_{0} \\ t_{0}\end{array}\right)$ to be

$$
\mathcal{B}_{r}(P)=\left\{\left(\begin{array}{l}
x \\
y \\
t
\end{array}\right): \begin{array}{c}
x \geq 0,\left|x-x_{0}\right| \leq r \\
t_{0}-r \leq t \leq y_{0} \mid \leq r
\end{array}\right\}
$$

and let $\mathcal{B}_{r}$ be the box around the point $P=\left(\begin{array}{l}0 \\ 0 \\ 1\end{array}\right)$. We have the following Theorem: 
II.1.2. Theorem. Assume that the coefficients $a^{i j}, b^{i}$ and $c$ of the operator $L$ belong to the space $C_{s}^{\alpha}\left(\mathcal{B}_{1}\right)$, for some number $\alpha$ in $0<\alpha<1$ and satisfy

$$
a^{i j} \xi_{i} \xi_{j} \geq \lambda|\xi|^{2}>0 \quad \forall \xi \in \mathcal{R}^{2} \backslash\{0\}
$$

and

$$
\left\|a^{i j}\right\|_{C_{s}^{\alpha}\left(Q_{T}\right)}, \quad\left\|b^{i}\right\|_{C_{s}^{\alpha}\left(Q_{T}\right)}, \quad\|c\|_{C_{s}^{\alpha}\left(Q_{T}\right)} \leq 1 / \lambda
$$

and

$$
b^{1} \geq \nu>0 \quad \text { at } \quad x=0
$$

for some positive constants $\lambda$ and $\nu$. Then, there exists a constant $C$ depending only on $\alpha, \lambda$, $\nu$ such that

$$
\|f\|_{C_{s}^{2+\alpha}\left(\mathcal{B}_{1 / 2}\right)} \leq C\left(\|f\|_{C_{s}^{\circ}\left(\mathcal{B}_{1}\right)}+\|L f\|_{C_{s}^{\alpha}\left(\mathcal{B}_{1}\right)}\right)
$$

for all functions $f \in C_{s}^{2+\alpha}\left(\mathcal{B}_{1}\right)$.

Proof. We will assume that $f$ is a $C^{\infty}$ function on $\mathcal{B}_{1}$. The case $f \in C_{s}^{2+\alpha}\left(\mathcal{B}_{1}\right)$ will then follow via a standard approximation argument, using the smoothing operators introduced in Section I.11.

Choose a bump function $\varphi$ so that

$$
\left\{\begin{array}{lll}
\varphi=1 & \text { on } & \mathcal{B}_{1 / 2} \\
\varphi=0 & \text { off } & \mathcal{B}_{1}
\end{array}\right.
$$

Then, the smooth function $\varphi f$, which vanishes off $\mathcal{B}_{1}$, satisfies the equation

$$
L(\varphi f)=\varphi L f+[L, \varphi] f
$$

with $[L, \varphi]$ denoting the commutator of $L$ and $\varphi$. Therefore, according to Theorem II.1.1, $\varphi f$ satisfies the estimate

$$
\|\varphi f\|_{C_{s}^{2+\alpha}\left(\mathcal{B}_{1}\right)} \leq C\left(\|\varphi L f\|_{C_{s}^{\alpha}\left(\mathcal{B}_{1}\right)}+\|[L, \varphi] f\|_{C_{s}^{\alpha}\left(\mathcal{B}_{1}\right)}\right) .
$$

The commutator $[L, \varphi]$ is only of first order and can be estimated, as in the proof of Theorem II.1.1, by standard interpolation between Hölder spaces. Indeed, for any number $\epsilon>0$ we have

$$
\|[L, \varphi] f\|_{C_{s}^{\alpha}\left(\mathcal{B}_{1}\right)} \leq \epsilon\|f\|_{C_{s}^{2+\alpha}\left(\mathcal{B}_{1}\right)}+C(\epsilon)\|f\|_{C^{0}\left(\mathcal{B}_{1}\right)} .
$$

Therefore, choosing $\epsilon$ sufficiently small and remembering that $\varphi \equiv 1$ on $\mathcal{B}_{1 / 2}$, we obtain

$$
\|f\|_{C_{s}^{2+\alpha}\left(\mathcal{B}_{1 / 2}\right)} \leq C\left(\|L f\|_{C_{s}^{\alpha}\left(\mathcal{B}_{1}\right)}+\|f\|_{C^{\circ}\left(\mathcal{B}_{1}\right)}\right)
$$

as desired.

The next result follows from the Schauder estimate above via a standard rescaling argument.

II.1.3. Theorem. Under the same hypotheses as in Theorem II.1.2 and for any number $r \leq 1$ there exists a constant $C(r)$ so that

$$
\|f\|_{C_{s}^{2+\alpha}\left(\mathcal{B}_{r / 2}\right)} \leq C(r)\left(\|f\|_{C_{s}^{\circ}\left(\mathcal{B}_{r}\right)}+\|L f\|_{C_{s}^{\alpha}\left(\mathcal{B}_{r}\right)}\right) .
$$


II.2. The quasilinear case. In this section we will the study quasilinear degenerate equations of the form

$$
w_{t}=\vartheta F^{i j}(t, x, y, w, D w) w_{i j}+G(t, x, y, w, D w)
$$

on the cylinder $Q_{T}=\Omega \times[0, T], T>0$. Let us denote by $P$ the operator

$$
P w=\vartheta F^{i j}(t, x, y, w, D w) w_{i j}+G(t, x, y, w, D w)
$$

and by $M$ the operator

$$
M w=w_{t}-P w .
$$

Then, if $\bar{w}$ is a fixed point in $C_{s}^{2+\alpha}\left(Q_{T}\right)$, the linearization of the operator $M$ at the point $\bar{w}$ is the operator

$$
\widetilde{M}(\tilde{w})=D M(\bar{w})(\tilde{w})=\tilde{w}_{t}-D P(\bar{w})(\tilde{w})
$$

with

$$
\begin{aligned}
D P(\bar{w})(\tilde{w})= & \vartheta F^{i j}(t, x, y, \bar{w}, D \bar{w}) \tilde{w}_{i j} \\
& +\left[\vartheta F_{w_{l}}^{i j}(t, x, y, \bar{w}, D \bar{w}) \bar{w}_{i j}+G_{w_{l}}(t, x, y, \bar{w}, D \bar{w})\right] \tilde{w}_{l} \\
& +\left[\vartheta F_{w}^{i j}(t, x, y, \bar{w}, D \bar{w}) \bar{w}_{i j}+G_{w}(t, x, y, \bar{w}, D \bar{w})\right] \tilde{w} .
\end{aligned}
$$

Here $D w=\left(w_{1}, w_{2}\right), F_{w}^{i j}=\partial F^{i j} / \partial w, G_{w}=\partial G / \partial w$ and for $l=1,2, F_{w_{l}}^{i j}=$ $\partial F^{i j} / \partial w_{l}, G_{w_{l}}=\partial G / \partial w_{l}$. As always, the summation convention is used. We have the following Theorem:

II.2.1. Theorem. Assume that $\Omega$ is a compact domain in $\mathcal{R}^{2}$ with smooth boundary, let $k$ be a nonnegative integer, and let $0<\alpha<1, T>0$ be positive numbers. Also, let $w^{0}$ be a function in $C_{s}^{k, 2+\alpha}(\Omega)$. Assume that the linearization $D M(\bar{w})$ of the quasilinear operator

$$
M w=w_{t}-\vartheta F^{i j}(t, x, y, w, D w) w_{i j}-G(t, x, y, w, D w)
$$

defined on $Q_{T}=\Omega \times[0, T]$, satisfies the hypotheses of Theorem II.1.1 at all points $\bar{w} \in C_{s}^{k, 2+\alpha}\left(Q_{T}\right)$, such that $\left\|\bar{w}-w^{0}\right\|_{C_{s}^{k, 2+\alpha}\left(Q_{T}\right)} \leq \mu, \mu>0$. Then, there exists a number $\tau_{0}$ in $0<\tau_{0} \leq T$ depending on the constants $\alpha, k, \lambda, \nu$ and $\mu$, for which the initial value problem

$$
\begin{cases}w_{t}=\vartheta F^{i j}(t, x, y, w, D w) w_{i j}+G(t, x, y, w, D w) & \text { in } \Omega \times\left[0, \tau_{0}\right], \\ w(\cdot, 0)=w^{0} & \text { on } \Omega\end{cases}
$$

admits a solution $w$ in the space $C_{s}^{k, 2+\alpha}\left(\Omega \times\left[0, \tau_{0}\right]\right)$. Moreover,

$$
\|w\|_{C_{s}^{k, 2+\alpha}\left(\Omega \times\left[0, \tau_{0}\right]\right)} \leq C\left\|w^{0}\right\|_{C_{s}^{k, 2+\alpha}(\Omega)}
$$

for some positive constant $C$ which depends only on $\alpha, k, \lambda, \nu$ and $\sigma$.

Proof. For any number $0<\tau \leq T$, we define the operator

$$
\bar{M}: C_{s}^{k, 2+\alpha}(\Omega \times[0, \tau]) \rightarrow C_{s}^{k, \alpha}(\Omega \times[0, \tau]) \times C_{s}^{k, 2+\alpha}(\Omega)
$$

by

$$
\bar{M} w=\left(M w, w_{t=0}\right) .
$$


Our assumptions imply that $\bar{M}$ is a well defined bounded operator. We will show that if $\tau$ is sufficiently small, there exists a function $w \in C_{s}^{k, 2+\alpha}(\Omega \times[0, \tau])$ and a function $g \in C_{s}^{k, \alpha}(\Omega \times[0, \tau])$ such that

$$
\bar{M}(w)=\left(g, w^{0}\right) \quad \text { and } \quad g(\cdot, t) \equiv 0, \quad \forall t \in\left[0, \tau_{0}\right]
$$

for some number $\tau_{0} \in(0, \tau]$, depending only on absolute constants. This will imply that our initial value problem is solvable on $\Omega \times\left[0, \tau_{0}\right]$, finishing the proof of the Theorem.

As before, we denote by $P(w)$ the operator

$$
P(w)=\vartheta F^{i j}(t, x, y, w, D w) w_{i j}+G(t, x, y, w, D w) .
$$

Since $P\left(w^{0}\right)$ belongs to the space $C_{s}^{k, \alpha}(\Omega)$, by Theorem I.11.7, there exists a function $\bar{w}$ in the space $C_{s}^{k, 2+\alpha}(\Omega \times[0, \tau])$ such that

$$
\bar{w}=w^{0} \quad \text { and } \quad \bar{w}_{t}=P\left(w^{0}\right) \quad \text { at } \quad t=0 .
$$

It follows that the function

$$
\bar{g}=\bar{w}_{t}-P(\bar{w})
$$

belongs to the space $C_{s}^{k, \alpha}(\Omega \times[0, \tau])$ and satisfies

$$
g(\cdot, t) \equiv 0 \quad \text { at } t=0 .
$$

We will prove, using the Inverse Function Theorem, that the operator $\bar{M}$ is invertible in a small neighborhood

$$
\|g-\bar{g}\|_{C_{s}^{k, \alpha}(\Omega \times[0, \tau])} \leq \epsilon, \quad\left\|w_{t=0}-w^{0}\right\|_{C_{s}^{k, \alpha}(\Omega)} \leq \epsilon
$$

of the point $\left(\bar{g}, w^{0}\right)$. For this purpose it is enough to show that the derivative of the operator $M$ at $\bar{w}$,

$$
D \bar{M}(\bar{w}): C_{s}^{k, 2+\alpha}(\Omega \times[0, \tau]) \rightarrow C_{s}^{k, \alpha}(\Omega \times[0, \tau]) \times C_{s}^{k, 2+\alpha}(\Omega)
$$

defined by

$$
D \bar{M}(\bar{w})(\tilde{w})=\left(D M(\bar{w})(\tilde{w}), \tilde{w}_{t=0}\right),
$$

is an invertible linear map. But this follows immediately from Theorem II.1.1, since the linear operator $D M(\bar{w})$ satisfies the required hypotheses. The same Theorem shows that

$$
\|\tilde{w}\|_{C_{s}^{k, 2+\alpha}(\Omega \times[0, \tau])} \leq C\left(\|D M(\bar{w}) \tilde{w}\|_{C_{s}^{k, \alpha}(\Omega \times[0, \tau])}+\left\|\tilde{w}_{t=0}\right\|_{C_{s}^{k, 2+\alpha}(\Omega)}\right)
$$

with $C$ a constant depending only on $\alpha, \lambda$ and $\nu$, if $\tau \leq 1$. Notice that the constant $C(T)$ in Theorem II.1.1 can be taken uniform in $T$, if $T \leq 1$.

On the other hand, since $\bar{g}(\cdot, t)=0$ at $t=0$, we can always find a function $g \in C_{s}^{k, \alpha}(\Omega \times[0, \tau])$ with $\|g-\bar{g}\|_{C_{s}^{k, \alpha}(\Omega \times[0, \tau])}<\epsilon$ and

$$
g(\cdot, t) \equiv 0, \quad \forall t \in\left[0, \tau_{0}\right]
$$

provided that $\tau_{0} \in(0, \tau]$ is sufficiently small, depending on $\epsilon$. Therefore there exists a function $w \in C^{k, 2+\alpha}\left(\Omega \times\left[0, \tau_{0}\right]\right)$ such that $M w=0$ in $\Omega \times\left[0, \tau_{0}\right]$ and $w(\cdot, 0)=w^{0}$. Moreover $w$ will satisfy the estimate

$$
\|w\|_{C_{s}^{k, 2+\alpha}\left(\Omega \times\left[0, \tau_{0}\right]\right)} \leq C\left\|w^{0}\right\|_{C_{s}^{k, 2+\alpha}(\Omega)}
$$

with $C$ a constant depending only $\alpha, k, \mu, \lambda, \nu$. 
PART III. The FREe BOUNDARY FOR THE POROUS MEDIUM EQUATION

III.1. The result. In this last part of the paper we will apply the results of Part II to study the regularity of the free boundary for the porous medium equation

$$
u_{t}=\Delta u^{m}
$$

with exponent $m>1$. Introducing the pressure

$$
f=m u^{m-1}
$$

we can express the porous medium equation in terms of $f$, namely

$$
f_{t}=f \Delta f+r(m)|D f|^{2}
$$

with

$$
r(m)=1 /(m-1)>0 .
$$

Let $\Omega$ be a compact domain in $\mathcal{R}^{2}$ and $f^{0}$ a function on $\Omega$ with $f^{0}=0$ at $\partial \Omega$ and $f^{0}>0$ in the interior of $\Omega$. We will study the free-boundary problem

$$
\begin{cases}f_{t}=f \Delta f+r|D f|^{2}, & (x, y, t) \in \Omega_{t} \times[0, T], \\ f(x, y, 0)=f^{0}, & (x, y) \in \Omega,\end{cases}
$$

for some $T>0$, where $\Omega_{t}$ is the closure of the set

$$
\left\{(x, y) \in \mathcal{R}^{2}: f(x, y, t)>0\right\}
$$

and $r$ is any positive number. Here and throughout the rest of the paper we will denote by $\Omega_{t} \times[0, T]$ the set

$$
\Omega_{t} \times[0, T]=\bigcup_{0 \leq t \leq T}\left(\Omega_{t} \times\{t\}\right) .
$$

Our main result is the following regularity theorem.

III.1.1. Theorem. Let $\Omega$ be a compact domain in $\mathcal{R}^{2}$ and let $f^{0}$ be a function in the space $C_{s}^{2+\alpha}(\Omega)$, for some $0<\alpha<1$, with $f^{0}=0$ at $\partial \Omega$ and $f^{0}>0$ in the interior of $\Omega$. Moreover, assume that

$$
D f^{0}(x, y) \neq 0, \quad \forall(x, y) \in \Omega .
$$

Then, there exists a number $T>0$, for which the free-boundary problem

$$
\begin{cases}f_{t}=f \Delta f+r|D f|^{2}, & (x, y, t) \in \Omega_{t} \times[0, T], \\ f(x, y, 0)=f^{0}, & (x, y) \in \Omega,\end{cases}
$$

with $r>0$, admits a solution $f$ which is smooth up to the free boundary $\partial \Omega_{t} \times(0, T]$. In particular, the interface $\partial \Omega_{t} \times(0, T]$ is smooth.

The Theorem stated in the Introduction is an immediate consequence of Theorem III.1.1, since $C^{\alpha}(\Omega) \subset C_{s}^{\alpha}(\Omega)$, for all $\alpha>0$. Notice that the solution of the free boundary problem in Theorem III.1.1, extended to be equal to zero on $\left(\mathcal{R}^{2} \backslash \Omega_{t}\right) \times$ $[0, T]$, is nothing but the unique weak solution of the Cauchy problem

$$
\begin{cases}f_{t}=f \Delta f+r|D f|^{2}, & (x, y, t) \in \mathcal{R}^{2} \times[0, T], \\ f(x, y, 0)=f^{0}, & (x, y) \in \mathcal{R}^{2} .\end{cases}
$$


III.2. Local coordinate change. To motivate the proof of the regularity Theorem III.1.1 we will first compute the transformation of the equation of the pressure

$$
f_{t}=f \Delta f+r|D f|^{2}
$$

when one exchanges dependent and independent variables near the boundary. This change of coordinates converts the free boundary into a fixed boundary. More precisely, assume for the moment that the function $f$ belongs to the space $C_{s}^{2+\alpha}\left(\Omega_{t} \times[0, T]\right)$. Pick a point $P_{0}=\left(x_{0}, y_{0}, t_{0}\right)$ at the free boundary $\partial \Omega_{t} \times(0, T]$. We can assume (by rotating the coordinates) that

$$
f_{x}\left(P_{0}\right)>0, \quad f_{y}\left(P_{0}\right)=0, \quad f_{t}\left(P_{0}\right)=r f_{x}^{2}\left(P_{0}\right)>0 .
$$

For a positive number $\delta$ sufficiently small, we denote by $Q_{\delta}$ the cube

$$
\left|x-x_{0}\right| \leq \delta, \quad\left|y-y_{0}\right| \leq \delta, \quad-\delta \leq t-t_{0} \leq 0,
$$

and by $\Lambda_{\delta}$, the intersection

$$
\Lambda_{\delta}=\left(\Omega_{t} \times[0, T]\right) \cap Q_{\delta} .
$$

Since the first derivatives of $f$ are Hölder continuous up to the free boundary, there exists a number $\delta>0$ such that

$$
f_{x}(x, y, t)>0, \quad f_{y}(x, y, t) \sim 0, \quad f_{t}(x, y, t)>0, \quad \forall(x, y, t) \in \Lambda_{\delta} .
$$

It follows from the Implicit Function Theorem that if the number $\delta$ is sufficiently small, we can solve the equation $z=f(x, y, t)$, for $(x, y, t) \in \Lambda_{\delta}$ with respect to $x$, yielding to a map

$$
x=h(z, y, t)
$$

defined for all $(z, y, t)$ sufficiently close to the point $\left(0, y_{0}, t_{0}\right)$. Also, after a moment of thought we can see that there exists a number $\eta>0$ sufficiently small, so that $h$ is defined on the cube

$$
\mathcal{B}_{\eta}=\left\{0 \leq z \leq \eta,\left|y-y_{0}\right| \leq \eta,-\eta \leq t-t_{0} \leq 0\right\} .
$$

We wish to find the differential equation satisfied by the function $h$ in $\mathcal{B}_{\eta}$. Notice that the free boundary $f=0$ has now been transformed into the fixed boundary $z=0$. We can easily compute the transformation of the first order derivatives by the equations

$$
h_{z} f_{x}=1, \quad h_{z} f_{t}+h_{t}=0, \quad h_{z} f_{y}+h_{y}=0
$$

which yield to

$$
f_{x}=\frac{1}{h_{z}}, \quad f_{t}=-\frac{h_{t}}{h_{z}}, \quad f_{y}=-\frac{h_{y}}{h_{z}} .
$$

We can differentiate once more to compute the transformation of the second order derivatives. We have

$$
h_{z z} f_{x}^{2}+h_{z} f_{x x}=0
$$

and

$$
h_{z z} f_{y}^{2}+2 h_{z y} f_{y}+h_{y y}+h_{z} f_{y y}=0 .
$$

Hence

$$
f_{x x}=-\frac{f_{x}^{2}}{h_{z}} h_{z z}=-\frac{1}{h_{z}^{3}} h_{z z}
$$


and

$$
f_{y y}=-\frac{1}{h_{z}}\left(f_{y}^{2} h_{z z}+2 f_{y} h_{z y}+h_{y y}\right)=-\frac{1}{h_{z}}\left(\frac{h_{y}^{2}}{h_{z}^{2}} h_{z z}-2 \frac{h_{y}}{h_{z}} h_{z y}+h_{y y}\right) .
$$

Notice that the function $h$ belongs to the space $C_{s}^{2+\alpha}\left(\mathcal{B}_{\eta}\right)$, since we have assumed that $f \in C_{s}^{2+\alpha}\left(\Omega_{t} \times[0, T]\right)$. This follows immediately from the above computations. Via this change of coordinates, the equation

$$
f_{t}-f\left(f_{x x}+f_{y y}\right)-r\left(f_{x}^{2}+f_{y}^{2}\right)=0
$$

transforms into the equation

$$
M(h)=h_{t}-z\left(\frac{1+h_{y}^{2}}{h_{z}^{2}} h_{z z}-\frac{2 h_{y}}{h_{z}} h_{z y}+h_{y y}\right)+r \frac{1+h_{y}^{2}}{h_{z}}=0 .
$$

The operator $M$ defined above is a quasilinear operator which becomes degenerate when $z=0$. We can easily compute its linearization $D M(h)$ at the point $h$ :

$$
\begin{aligned}
D M(h)(\tilde{h})= & \tilde{h}_{t}-z\left(\frac{1+h_{y}^{2}}{h_{z}^{2}} \tilde{h}_{z z}-\frac{2 h_{y}}{h_{z}} \tilde{h}_{z y}+\tilde{h}_{y y}\right) \\
& -\left(r \frac{1+h_{y}^{2}}{h_{z}^{2}}-2 z \frac{1+h_{y}^{2}}{h_{z}^{3}} h_{z z}+2 \frac{z h_{y} h_{z y}}{h_{z}^{2}}\right) \tilde{h}_{z} \\
& +\left(2 r \frac{h_{y}}{h_{z}}-2 z \frac{h_{y}}{h_{z}^{2}} h_{z z}+\frac{2 z h_{z y}}{h_{z}}\right) \tilde{h}_{y} .
\end{aligned}
$$

Since $h \in C_{s}^{2+\alpha}\left(\mathcal{B}_{\eta}\right)$ and on the set $\mathcal{B}_{\eta}$ we have

$$
h_{z}(z, y, t)>0, \quad h_{y}(x, y, t) \sim 0
$$

the linearized operator $D M(h)$ on $\mathcal{B}_{\eta}$ belongs to the class of the degenerate operators studied in Part II.

Our goal is to use the results in Part II for the proof of Theorem III.1.1. However, the change of coordinates presented here is only local and can't be used directly for the proof of Theorem III.1.1. In the next section we will introduce a more subtle, global change of coordinates which is based on similar ideas.

III.3. Partial regularity. Let $\Omega$ be a compact domain in $\mathcal{R}^{2}$ and $f^{0}$ a function on $\Omega$ with $f^{0}=0$ at $\partial \Omega$ and $f^{0}>0$ in the interior of $\Omega$. Assume that $f^{0} \in$ $C_{s}^{k, 2+\alpha}(\Omega)$, for some nonnegative integer $k$ and that

$$
\left|D f^{0}(x, y)\right|+f^{0}(x, y) \geq c>0 \quad \forall(x, y) \in \Omega
$$

for some fixed positive number $c$. Denote by $\mathcal{D}$ the unit disk

$$
\mathcal{D}=\left\{(u, v) \in \mathcal{R}^{2}: u^{2}+v^{2} \leq 1\right\}
$$

and pick a smooth surface $\mathcal{S}$, sufficiently close to the surface $z=f^{0}(x, y)$. Let $S: \mathcal{D} \rightarrow \mathcal{R}^{3}$ be a smooth parametrization for the surface $\mathcal{S}$ which maps $\partial \mathcal{D}$ onto $\mathcal{S} \cap\{z=0\}$. Also, let

$$
\mathcal{T}=\left(\begin{array}{c}
\mathcal{T}_{1} \\
\mathcal{T}_{2} \\
\mathcal{T}_{3}
\end{array}\right)
$$

be a smooth vector field, transverse to the surface $\mathcal{S}$. Since $\left|D f^{0}\right| \geq c$ along $\partial \Omega$ and $\mathcal{S}$ is sufficiently close to the surface $z=f^{0}(x, y)$, we can choose $\mathcal{T}$ to be parallel 
to the plane $z=0$ in a small neighborhood of $\partial \mathcal{D}$. In other words, there exists a number $\delta>0$ depending on $k$ such that

$$
\mathcal{T}_{3}\left(\begin{array}{l}
u \\
v
\end{array}\right)=0 \quad \text { on } \quad \mathcal{D} \backslash \mathcal{D}_{1-\delta}
$$

with $\mathcal{D}_{1-\delta}=\left\{(u, v) \in \mathcal{R}^{2}: u^{2}+v^{2} \leq 1-\delta\right\}$.

For $\eta>0$ sufficiently small, we define the change of spatial coordinates $\Phi$ : $\mathcal{D} \times[-\eta, \eta] \rightarrow \mathcal{R}^{3}$ by

$$
\left(\begin{array}{l}
x \\
y \\
z
\end{array}\right)=\Phi\left(\begin{array}{l}
u \\
v \\
w
\end{array}\right)=S\left(\begin{array}{l}
u \\
v
\end{array}\right)+w \mathcal{T}\left(\begin{array}{l}
u \\
v
\end{array}\right) .
$$

The map $\Phi$ defines $x, y$ and $z$ as functions of the new coordinates $u, v$ and $w$.

Assume that $z=f(x, y, t)$ satisfies the initial free-boundary problem

$$
\begin{cases}f_{t}=f \Delta f+r|D f|^{2}, & (x, y, t) \in \Omega_{t} \times[0, T], \\ f(x, y, 0)=f^{0}, & (x, y) \in \Omega,\end{cases}
$$

for some $r>0$, where $\Omega_{t}$ is the closure of the set $\left\{(x, y) \in \mathcal{R}^{2}: f(x, y, t)>0\right\}$. Under the coordinate change $\Phi$, the initial data $f^{0}(x, y)$ transforms to a function $w^{0}(u, v)$ which can be made arbitrarily small, by choosing the smooth surface $\mathcal{S}$ sufficiently close to the surface $z=f^{0}(x, y)$. We will see that $w^{0} \in C_{s}^{k, 2+\alpha}(\mathcal{D})$, since $f \in C_{s}^{k, 2+\alpha}(\Omega)$.

When $z$ evolves as a function of $(x, y, t)$, then, through this coordinate change $w$ evolves as a function of $(u, v, t)$ with $(u, v) \in \mathcal{D}$. Notice that by our choice of the parametrization $S$ we have $(u, v) \in \partial \mathcal{D}$ iff $z=0$. Hence the free-boundary where $z=f(x, y, t)=0$ is mapped onto the fixed lateral boundary of the cylinder $\mathcal{D} \times[0, T]$.

The evolution of $w$ is described in the following Theorem, where to simplify the notation we use subscripts $i, j, k \in\{u, v\}$ to denote differentiation with respect to the variables $u, v$ :

III.3.2. Theorem. Let $\Omega$ be a compact domain in $\mathcal{R}^{2}, k$ a nonnegative integer and let $f^{0}$ be a function in the space $C_{s}^{k, 2+\alpha}(\Omega)$, for some number $\alpha$ in $0<\alpha<1$. Assume that $f^{0}>0$ in the interior of $\Omega$ and $f^{0}=0$ at $\partial \Omega$ with

$$
\left|D f^{0}(x, y)\right|+f^{0}(x, y) \geq c \quad \forall(x, y) \in \Omega .
$$

Then, under the coordinate change (III.3.1) the initial free boundary problem

$$
\begin{cases}f_{t}=f \Delta f+r|D f|^{2}, & (x, y, t) \in \Omega_{t} \times[0, T], \\ f(x, y, 0)=f^{0}, & (x, y) \in \Omega,\end{cases}
$$

with $r>0$, converts into the initial value problem

$$
\begin{cases}M w=0, & (u, v, t) \in \mathcal{D} \times[0, T], \\ w(u, v, 0)=w^{0}, & (u, v) \in \mathcal{D},\end{cases}
$$

with

$$
M w=w_{t}-\left(\vartheta F^{i j}(t, u, v, w, D w) w_{i j}+G(t, u, v, w, D w)\right)
$$

and $w^{0} \in C_{s}^{k, 2+\alpha}(\mathcal{D})$. Moreover, if $T \leq \tau_{k}$, with $\tau_{k}$ sufficiently small depending on $c$ and $k$, the operator $M$ satisfies all the hypotheses of Theorem II.2.1. 
Proof. We begin by selecting a sufficiently small number $\delta>0$, such that

$$
\mathcal{T}_{3}\left(\begin{array}{l}
u \\
v
\end{array}\right)=0 \quad \text { on } \quad \mathcal{D} \backslash \mathcal{D}_{1-\delta}
$$

with $\mathcal{T}=\left(\begin{array}{c}\mathcal{T}_{1} \\ \mathcal{T}_{2} \\ \mathcal{T}_{3}\end{array}\right)$ denoting, as above, the transverse vector field to the surface $\mathcal{S}$. Notice that by choosing the smooth surface sufficiently close to the surface $z=f^{0}(x, y)$, we can make $\delta$ depend only on the constant $c$.

To show that $w$ satisfies an equation of the desired form in the interior cylinder $\mathcal{D}_{1-\delta} \times[0, T]$ is straightforward. Hence, we will restrict our attention to $\mathcal{D} \backslash \mathcal{D}_{1-\delta}$. We start by expressing the first and second derivatives of $z$ with respect to $x, y, t$ in terms of the first and second derivatives of $w$ with respect to $u, v, t$.

We begin with the first order derivatives. Since $x, y$ and $z$ are functions of $u, v$ and $w$, while $w$ is a function of $u, v$ and $t$ we have

$$
\left(\begin{array}{ll}
\frac{\partial x}{\partial u} & \frac{\partial y}{\partial u} \\
\frac{\partial x}{\partial v} & \frac{\partial y}{\partial v}
\end{array}\right)=\left(\begin{array}{ll}
x_{u}+x_{w} \frac{\partial w}{\partial u} & y_{u}+y_{w} \frac{\partial w}{\partial u} \\
x_{v}+x_{w} \frac{\partial w}{\partial v} & y_{v}+y_{w} \frac{\partial w}{\partial v}
\end{array}\right)
$$

where $x_{u}, y_{u}, z_{u}, x_{v}, y_{v}, z_{v}$ and $x_{w}, y_{w}, z_{w}$, denote the partial derivatives of the functions $x=x(u, v, w), y=y(u, v, w)$ and $z=z(u, v, w)$ with respect to $u, v, w$ respectively.

Therefore we can compute the partial derivatives of the functions $u=u(x, y, t)$ and $v=v(x, y, t)$ by

$$
\left(\begin{array}{ll}
\frac{\partial u}{\partial x} & \frac{\partial v}{\partial x} \\
\frac{\partial u}{\partial y} & \frac{\partial v}{\partial y}
\end{array}\right)=\left(\begin{array}{ll}
\frac{\partial x}{\partial u} & \frac{\partial y}{\partial u} \\
\frac{\partial x}{\partial v} & \frac{\partial y}{\partial v}
\end{array}\right)^{-1}=\frac{1}{D}\left(\begin{array}{cc}
\frac{\partial y}{\partial v} & -\frac{\partial y}{\partial u} \\
-\frac{\partial x}{\partial v} & \frac{\partial x}{\partial u}
\end{array}\right)
$$

with

$$
D=\frac{\partial x}{\partial u} \frac{\partial y}{\partial v}-\frac{\partial x}{\partial v} \frac{\partial y}{\partial u}
$$

Since for $u^{2}+v^{2} \geq 1-\delta$ the coordinate $z$ is independent of $w$ because $T_{3}=0$, we have

$$
\left(\begin{array}{l}
\frac{\partial z}{\partial x} \\
\frac{\partial z}{\partial y}
\end{array}\right)=\left(\begin{array}{c}
z_{u} \frac{\partial u}{\partial x}+z_{v} \frac{\partial v}{\partial x} \\
z_{u} \frac{\partial u}{\partial y}+z_{v} \frac{\partial v}{\partial y}
\end{array}\right)
$$

and hence from (III.3.4)

$$
\left(\begin{array}{l}
\frac{\partial z}{\partial x} \\
\frac{\partial z}{\partial y}
\end{array}\right)=\frac{1}{D}\left(\begin{array}{cc}
\frac{\partial y}{\partial v} & -\frac{\partial y}{\partial u} \\
-\frac{\partial x}{\partial v} & \frac{\partial x}{\partial u}
\end{array}\right)\left(\begin{array}{c}
z_{u} \\
z_{v}
\end{array}\right) .
$$

We compute next the transformation of the second order derivatives. Differentiating the function $z$ in (III.3.5) once more with respect to $x$ and $y$ and summing 
up we obtain

$$
\begin{aligned}
\frac{\partial^{2} z}{\partial x^{2}}+\frac{\partial^{2} z}{\partial y^{2}} & =z_{u}\left(\frac{\partial^{2} u}{\partial x^{2}}+\frac{\partial^{2} u}{\partial y^{2}}\right)+z_{v}\left(\frac{\partial^{2} v}{\partial x^{2}}+\frac{\partial^{2} v}{\partial y^{2}}\right) \\
+\left\{z_{u u}\left[\left(\frac{\partial u}{\partial x}\right)^{2}+\left(\frac{\partial u}{\partial y}\right)^{2}\right]+2 z_{u v}\left[\frac{\partial u}{\partial x} \frac{\partial v}{\partial x}+\frac{\partial u}{\partial y} \frac{\partial v}{\partial y}\right]\right. & \left.+z_{v v}\left[\left(\frac{\partial v}{\partial x}\right)^{2}+\left(\frac{\partial v}{\partial y}\right)^{2}\right]\right\}
\end{aligned}
$$

The second derivatives $\partial^{2} u / \partial x^{2}$ and $\partial^{2} v / \partial x^{2}$ satisfy the linear system

$$
\left(\begin{array}{cc}
\frac{\partial x}{\partial u} & \frac{\partial x}{\partial v} \\
\frac{\partial y}{\partial u} & \frac{\partial y}{\partial v}
\end{array}\right)\left(\begin{array}{c}
\frac{\partial^{2} u}{\partial x^{2}} \\
\frac{\partial^{2} v}{\partial x^{2}}
\end{array}\right)+\left(\begin{array}{l}
\frac{\partial^{2} x}{\partial u^{2}}\left(\frac{\partial u}{\partial x}\right)^{2}+2 \frac{\partial^{2} x}{\partial u \partial v} \frac{\partial u}{\partial x} \frac{\partial v}{\partial x}+\frac{\partial^{2} x}{\partial v^{2}}\left(\frac{\partial v}{\partial x}\right)^{2} \\
\frac{\partial^{2} y}{\partial u^{2}}\left(\frac{\partial u}{\partial x}\right)^{2}+2 \frac{\partial^{2} y}{\partial u \partial v} \frac{\partial u}{\partial x} \frac{\partial v}{\partial x}+\frac{\partial^{2} y}{\partial v^{2}}\left(\frac{\partial v}{\partial x}\right)^{2}
\end{array}\right)=0
$$

while the derivatives $\partial^{2} u / \partial y^{2}$ and $\partial^{2} v / \partial y^{2}$ satisfy a similar system. After several simple calculations we conclude that

$$
\begin{aligned}
\frac{\partial^{2} z}{\partial x^{2}}+\frac{\partial^{2} z}{\partial y^{2}} & =\frac{1}{D^{3}}\left\{\left(z_{v} \frac{\partial y}{\partial u}-z_{u} \frac{\partial y}{\partial v}\right)\left(A \frac{\partial^{2} x}{\partial u^{2}}-2 B \frac{\partial^{2} x}{\partial u \partial v}+C \frac{\partial^{2} x}{\partial v^{2}}\right)\right. \\
& \left.+\left(z_{u} \frac{\partial x}{\partial v}-z_{v} \frac{\partial x}{\partial u}\right)\left(A \frac{\partial^{2} y}{\partial u^{2}}-2 B \frac{\partial^{2} y}{\partial u \partial v}+C \frac{\partial^{2} y}{\partial v^{2}}\right)\right\} \\
& +\frac{1}{D^{2}}\left(A z_{u u}-2 B z_{u v}+C z_{v v}\right)
\end{aligned}
$$

where, to simplify the notation, we have set

$$
A=\left(\frac{\partial y}{\partial v}\right)^{2}+\left(\frac{\partial x}{\partial v}\right)^{2}, \quad B=\frac{\partial y}{\partial v} \frac{\partial y}{\partial u}+\frac{\partial x}{\partial v} \frac{\partial x}{\partial u}, \quad C=\left(\frac{\partial y}{\partial u}\right)^{2}+\left(\frac{\partial x}{\partial u}\right)^{2}
$$

On the other hand, differentiating (III.3.3) we obtain

$$
\frac{\partial^{2} x}{\partial u^{2}}=x_{u u}+2 x_{u w} \frac{\partial w}{\partial u}+x_{w} \frac{\partial^{2} w}{\partial u^{2}}, \quad \frac{\partial^{2} x}{\partial v^{2}}=x_{v v}+2 x_{v w} \frac{\partial w}{\partial v}+x_{w} \frac{\partial^{2} w}{\partial v^{2}}
$$

and

$$
\frac{\partial^{2} x}{\partial u \partial v}=x_{u v}+x_{u w} \frac{\partial w}{\partial v}+x_{v w} \frac{\partial w}{\partial u}+x_{w} \frac{\partial^{2} w}{\partial u \partial v}
$$

while we have similar expressions for the derivatives $\partial^{2} y / \partial u^{2}, \partial^{2} y / \partial v^{2}$ and $\partial^{2} y / \partial u \partial v$.

Using the above computations we now find that

$$
\begin{aligned}
\frac{\partial^{2} z}{\partial x^{2}}+\frac{\partial^{2} z}{\partial y^{2}}= & -\frac{1}{D^{3}}\left(x_{w} K+y_{w} M\right)\left(A \frac{\partial^{2} w}{\partial u^{2}}-2 B \frac{\partial^{2} w}{\partial u \partial v}+C \frac{\partial^{2} w}{\partial v^{2}}\right) \\
& -\frac{2}{D^{3}}\left\{\left[K\left(A x_{u w}-B x_{v w}\right)+M\left(A y_{u w}-B y_{v w}\right)\right] \frac{\partial w}{\partial u}\right. \\
& \left.-\left[K\left(C x_{v w}-B x_{u w}\right)+M\left(C y_{v w}-B y_{u w}\right)\right] \frac{\partial w}{\partial v}\right\} \\
& -\frac{1}{D^{3}}\left[K\left(A x_{u u}-2 B x_{u v}+C x_{v v}\right)+M\left(A y_{u u}-2 B y_{u v}+C y_{v v}\right)\right]
\end{aligned}
$$


where, to simplify the notation, we have set

$$
K=z_{u} \frac{\partial y}{\partial v}-z_{v} \frac{\partial y}{\partial u}, \quad M=z_{v} \frac{\partial x}{\partial u}-z_{u} \frac{\partial x}{\partial v} .
$$

Notice that under this notation we have

$$
\frac{\partial z}{\partial x}=\frac{K}{D} \quad \text { and } \quad \frac{\partial z}{\partial y}=\frac{M}{D} .
$$

Finally, to compute the transformation of the time derivative $\partial z / \partial t$, we differentiate $z=f(x, y, t)$ with respect to time $t$. We find

$$
\frac{\partial z}{\partial t}+\left(x_{w} \frac{\partial z}{\partial x}+y_{w} \frac{\partial z}{\partial y}\right) \frac{\partial w}{\partial t}=0
$$

which gives

$$
\frac{\partial w}{\partial t}=-\frac{D}{x_{w} K+y_{w} M} \frac{\partial z}{\partial t} .
$$

We are now ready to compute the evolution equation of $w$ on $\left(D \backslash D_{\delta}\right) \times[0, T]$. Since $z=f(x, y, t)$ evolves as

$$
f_{t}=f\left(f_{x x}+f_{y y}\right)+r\left(f_{x}^{2}+f_{y}^{2}\right)
$$

from the above computations we conclude that $w$ evolves as

$$
\begin{aligned}
\frac{\partial w}{\partial t} & =\frac{z}{D^{2}}\left(A \frac{\partial^{2} w}{\partial u^{2}}-2 B \frac{\partial^{2} w}{\partial u \partial v}+C \frac{\partial^{2} w}{\partial v^{2}}\right) \\
& +\frac{2 z}{D^{2}\left(x_{w} K+y_{w} M\right)}\left(\left[K\left(A x_{u w}-B x_{v w}\right)+M\left(A y_{u w}-B y_{v w}\right)\right] \frac{\partial w}{\partial u}\right. \\
& \left.+\left[K\left(C x_{v w}-B x_{u w}\right)+M\left(C y_{v w}-B y_{u w}\right)\right] \frac{\partial w}{\partial v}\right) \\
& +\frac{z}{D^{2}\left(x_{w} K+y_{w} M\right)}\left\{K\left(A x_{u u}-2 B x_{u v}+C x_{v v}\right)+M\left(A y_{u u}-2 B y_{u v}+C y_{v v}\right)\right\} \\
& -\frac{r}{D\left(x_{w} K+y_{w} M\right)}\left(K^{2}+M^{2}\right) .
\end{aligned}
$$

Next notice that on $\mathcal{D} \backslash \mathcal{D}_{\delta}$ the $z$ coordinate in (III.3.1) is independent of $w\left(\mathcal{T}_{3}=0\right)$. In other words $z=S_{3}$, with $S_{3}$ denoting the $z$ coordinate of the parametrization $S$. Therefore, since $S_{3}$ maps $\partial \mathcal{D}$ onto $z=0$, we can write $z=\vartheta(u, v)$ on $\mathcal{D} \backslash \mathcal{D}_{\delta}$, where $\vartheta$ is a smooth function, strictly positive in the interior of $\mathcal{D}$ and such that

$$
\vartheta(u, v) \sim d(u, v) \quad \text { on } \quad \mathcal{D} \backslash \mathcal{D}_{1-\delta}
$$

with $d(u, v)$ denoting the distance of the point $(u, v)$ to the boundary of $\mathcal{D}$.

We can rewrite the equation of $w$ on $\left(\mathcal{D} \backslash \mathcal{D}_{1-\delta}\right) \times[0, T]$ as

$$
\begin{aligned}
\frac{\partial w}{\partial t}= & \frac{\vartheta}{D^{2}}\left(A \frac{\partial^{2} w}{\partial u^{2}}-2 B \frac{\partial^{2} w}{\partial u \partial v}+C \frac{\partial^{2} w}{\partial v^{2}}\right) \\
& -\frac{r\left(K^{2}+M^{2}\right)}{D\left(x_{w} K+y_{w} M\right)}+\vartheta H(t, u, v, w, D w) .
\end{aligned}
$$

It is then easy to see that the equation takes the form

$$
w_{t}-\left(\vartheta F^{i j}(t, u, v, w, D w) w_{i j}+G(t, u, v, w, D w)\right)=0 .
$$


The subindices $i, j$ denote differentiation with respect to the variables $u, v$ respectively. As always the summation convention is used. Under this notation

$$
F^{i i}=\frac{A}{D^{2}}, \quad F^{i j}=F^{j i}=-\frac{B}{D^{2}}, \quad F^{j j}=\frac{C}{D^{2}}
$$

and

$$
G(t, u, v, w, D w)=-\frac{r\left(K^{2}+M^{2}\right)}{D\left(x_{w} K+y_{w} M\right)}+\vartheta H(t, u, v, w, D w) .
$$

The coordinate change $\Phi$ in (III.3.1) transforms the initial data $f^{0}(x, y)$ into a function $w^{0}(u, v)$. The reader can verify that if $f^{0} \in C_{s}^{k, 2+\alpha}(\Omega)$, then $w^{0} \in$ $C_{s}^{k, 2+\alpha}(\mathcal{D})$. Moreover, the norm $\left\|w^{0}\right\|_{C_{s}^{k, 2+\alpha}(\mathcal{D})}$ can be made arbitrarily small, by choosing the smooth surface $\mathcal{S}$ in (III.3.1) sufficiently close to the surface $z=$ $f^{0}(x, y)$.

Since the change of variables $\Phi$ in (III.3.1) is smooth, the functions $F^{i j}$ and $G$ will depend smoothly on the variables $(t, u, v, w, D w)$ as long as $|D|>0$ and $\left|x_{w} K+y_{w} M\right|>0$ for all $(u, v) \in \mathcal{D} \backslash \mathcal{D}_{\delta}$. Therefore, in order to prove that the operator

$$
M w=w_{t}-\left(\vartheta F^{i j}(t, u, v, w, D w) w_{i j}+G(t, u, v, w, D w)\right)
$$

satisfies the hypotheses of Theorem II.2.1 on $\left(\mathcal{D} \backslash \mathcal{D}_{1-\delta}\right) \times[0, T]$, for $T$ sufficiently small, it will be enough to show that at $t=0$ and for $\delta$ small,

$$
|D|>0 \quad \text { and } \quad\left|x_{w} K+y_{w} M\right|>0 \quad \text { on } \mathcal{D} \backslash \mathcal{D}_{1-\delta}
$$

the matrix

$$
F^{i j}\left(t, u, v, w^{0}, D w^{0}\right)_{t=0}, \quad(u, v) \in \mathcal{D} \backslash \mathcal{D}_{1-\delta},
$$

is strictly positive, and also

$$
n_{l} \frac{\partial}{\partial w_{l}}\left(\frac{-r\left(K^{2}+M^{2}\right)}{D\left(x_{w} K+y_{w} M\right)}\right)_{t=0}\left(t, u, v, w^{0}, D w^{0}\right)>0 \quad \text { at } \partial \mathcal{D}
$$

and

$$
\vartheta\left(\frac{\partial H}{\partial w_{l}}\right)_{t=0}\left(t, u, v, w^{0}, D w^{0}\right)=0 \quad \text { at } \partial \mathcal{D} .
$$

Here $D w=\left(w_{u}, w_{v}\right), l \in\{u, v\}$ and $n=\left(n_{1}, n_{2}\right)$ denotes the interior normal to $\partial \mathcal{D}$. Once more the summation convention is used.

Choose a point $\left(u_{0}, v_{0}\right) \in \partial \mathcal{D}$ which is mapped, through our coordinate change $\Phi$, to the point $\left(x_{0}, y_{0}\right) \in \mathcal{S} \cap\{z=0\}$. We can assume, by rotating the $(u, v)$-axes, that $\left(u_{0}, v_{0}\right)$ is the point $(1,0)$. Moreover, we can rotate the $(x, y)$-axes so that the exterior normal to $\mathcal{S} \cap\{z=0\}$ at $\left(x_{0}, y_{0}\right)$ is parallel to the vector $(1,0)$. Therefore, at $\left(u_{0}, v_{0}\right)=(1,0)$ we have

$$
x_{u} \sim 1, \quad x_{v} \sim 0, \quad y_{u} \sim 0, \quad y_{v} \sim \pm 1
$$

in the coordinate change (III.3.1). Hence, for $w^{0} \sim 0, D w^{0} \sim 0$ (which holds true since $\left\|w^{0}\right\|_{C^{2+\alpha}(\mathcal{D})}$ can be made arbitrarily small) we compute

$$
\left(\begin{array}{ll}
\frac{\partial x}{\partial u} & \frac{\partial x}{\partial v} \\
\frac{\partial y}{\partial u} & \frac{\partial y}{\partial v}
\end{array}\right) \sim\left(\begin{array}{cc}
1 & 0 \\
0 & \pm 1
\end{array}\right)
$$


It is now easy to see that

$$
\left(\begin{array}{ll}
A & B \\
B & C
\end{array}\right) \sim\left(\begin{array}{ll}
1 & 0 \\
0 & 1
\end{array}\right)
$$

and

$$
D \sim y_{v} \sim \pm 1
$$

while

$$
K \sim z_{u} y_{v} \quad \text { and } \quad M \sim z_{v} .
$$

Since the exterior normal to $\mathcal{S} \cap\{z=0\}$ at $\left(x_{0}, y_{0}\right)$ is parallel to the vector $(1,0)$ and $\mathcal{S}$ can be chosen arbitrarily close to $z=f^{0}(x, y)$ we have

$$
\frac{\partial f^{0}}{\partial x}<0 \quad \text { and } \quad \frac{\partial f^{0}}{\partial y} \sim 0
$$

at the point $\left(x_{0}, y_{0}\right)$. Hence

$$
\frac{K}{D}=\frac{\partial z}{\partial x} \sim \frac{\partial f^{0}}{\partial x}<0 \quad \text { and } \quad \frac{M}{D}=\frac{\partial z}{\partial y} \sim \frac{\partial f^{0}}{\partial y} \sim 0
$$

at the point $(1,0)$. Therefore

$$
z_{u} \sim \frac{K}{D} \sim-1 \quad \text { and } \quad z_{v} \sim M \sim 0
$$

at the point $(1,0)$. We conclude that

$$
x_{w} K+y_{w} M \sim-1
$$

at the point $(1,0)$, since $x_{w}=T_{1} \sim 1$. Hence

$$
|D| \sim 1 \quad \text { and } \quad\left|x_{w} K+y_{w} M\right| \sim 1
$$

at $(1,0)$, as desired. Moreover, the matrix

$$
\left(F^{i j}\right)=\frac{1}{D^{2}}(\underset{B}{A} \underset{C}{B})
$$

is strictly positive at $t=0$ at the point $(1,0)$.

We will show next that at $t=0$ and at the point $(1,0)$ we have

$$
\frac{\partial}{\partial w_{u}}\left(\frac{K^{2}+M^{2}}{D\left(x_{w} K+y_{w} M\right)}\right)>0 .
$$

To simplify the notation we denote by $K_{u}, M_{u}, D_{u}$ the partial derivatives $\partial K / \partial w_{u}$, $\partial M / \partial w_{u}$ and $\partial D / \partial w_{u}$ respectively. The reader can easlily check that $x_{w} K+y_{w} M$ is independent of $w_{u}$. Hence

$$
\frac{\partial}{\partial w_{u}}\left(\frac{K^{2}+M^{2}}{D\left(x_{w} K+y_{w} M\right)}\right)=\frac{-\left(K^{2}+M^{2}\right) D_{u}}{D^{2}\left(x_{w} K+y_{w} M\right)}+\frac{2\left(K K_{u}+M M_{u}\right)}{D\left(x_{w} K+y_{w} M\right)}
$$

with

$$
D_{u}=x_{w}\left(y_{v}+y_{w} \frac{\partial w}{\partial v}\right)-y_{w}\left(x_{v}+x_{w} \frac{\partial w}{\partial v}\right)=x_{w} y_{v}-y_{w} x_{v} \sim x_{w} y_{v}
$$

and

$$
K_{u}=-z_{v} y_{w} \sim 0 \quad \text { and } \quad M_{u}=z_{v} x_{w} \sim 0,
$$


since $x_{v} \sim 0$ and $z_{v} \sim 0$ at the point $(1,0)$. Finally we conclude that

$$
\frac{\partial}{\partial w_{u}}\left(\frac{K^{2}+M^{2}}{D\left(x_{w} K+y_{w} M\right)}\right) \sim-\frac{K^{2}}{D^{2}} \frac{1}{x_{w} z_{u}} \sim 1
$$

since $K / D \sim-1, M \sim 0, z_{u} \sim-1$ and $x_{w}=T_{1} \sim 1$ at $(1,0)$. We conclude that

$$
n_{l} \frac{\partial}{\partial w_{l}}\left(\frac{-r\left(K^{2}+M^{2}\right)}{D\left(x_{w} K+y_{w} M\right)}\right) \sim 1
$$

at the point $(1,0)$, since the exterior normal $n=\left(n_{1}, n_{2}\right)$ at $(1,0)$ is $(1,0)$.

It remains to be shown that $\vartheta\left(\partial H / \partial w_{l}\right)=0$ at $\left(t, u, v, w^{0}, D w^{0}\right)$ for $t=0$ and $(u, v) \in \partial \mathcal{D}$. Since the coordinate change $\Phi$ is smooth and the function $w^{0}$ belongs to the space $C_{s}^{k, 2+\alpha}(\mathcal{D})$ while

$$
\vartheta(u, v) \sim d(u, v) \quad \text { as }(u, v) \rightarrow \partial \mathcal{D}
$$

with $d(u, v)$ denoting the distance to the boundary of $\mathcal{D}$, this follows as an immediate consequence from the next simple lemma.

III.3.6. Lemma. Let $\xi$ be a function in $C_{s}^{k, 2+\alpha}(\mathcal{D})$, for some nonnegative integer $k$ and some number $\alpha$ in $0<\alpha<1$. Then

$$
d D^{2} \xi=0 \quad \text { at } \partial \mathcal{D}
$$

with $d$ denoting the distance function to the boundary of $\mathcal{D}$.

Proof of Lemma. The lemma is obvious when $k>1$. Hence we can assume that $\xi \in C_{s}^{2+\alpha}(\mathcal{D})$. Pick a point $\left(u_{0}, v_{0}\right) \in \partial \mathcal{D}$, wich can be taken without loss of generality to be the point $(1,0)$. We will show that $d \xi_{u u}, d \xi_{u v}$ and $d \xi_{v v}$ are all equal to zero at $(1,0)$. We proceed by contradiction. Assume that $d \xi_{u u}=\alpha>0$ at $(1,0)$ (if $\alpha<0$ we replace $\xi$ by $-\xi$ ). Then by continuity

$$
\xi_{u u}(u, 0) \geq \frac{\alpha}{2(1-u)}>0
$$

for all $1-\epsilon \leq u \leq 1$, for some $\epsilon>0$. Hence, for $1-\epsilon<u_{1}<u_{2}<1$, we have

$$
\xi_{u}\left(u_{2}, 0\right)-\xi_{u}\left(u_{1}, 0\right)=\int_{u_{1}}^{u_{2}} \xi_{u u}(u, 0) d u \geq \int_{u_{1}}^{u_{2}} \frac{\alpha}{2(1-u)} d u \geq \frac{\alpha}{2} \ln \frac{1-u_{1}}{1-u_{2}} .
$$

Letting $u_{2} \rightarrow 1$ we derive a contradiction, since $\xi_{u}$ is bounded on $\mathcal{D}$. Assume next that $d \xi_{v v}=\alpha>0$ at $(0,1)$. Then, by continuity

$$
\xi_{v v}(u, v) \geq \frac{\alpha}{2 d}>0
$$

for all $(u, v) \in \mathcal{D}$ with $\sqrt{1-\epsilon^{2}} \leq u \leq 1$ and $-\epsilon \leq v \leq \epsilon$, for some $\epsilon>0$. Hence

$$
\xi_{v}\left(\sqrt{1-\epsilon^{2}}, \epsilon / 2\right)-\xi_{v}\left(\sqrt{1-\epsilon^{2}},-\epsilon / 2\right)=\int_{-\epsilon / 2}^{\epsilon / 2} \xi_{v v}\left(\sqrt{1-\epsilon^{2}}, v\right) d v \geq \frac{\alpha}{\epsilon} .
$$

Letting $\epsilon \rightarrow 0$ we derive a contradiction. At last the equality $d \xi_{u v}=0$ at $(1,0)$ can be proved in a similar manner. This finishes the proof of the lemma and also the proof of Theorem III.3.2.

As a consequence of Theorem III.3.2 and Theorem II.2.1, we obtain the following main result. 
III.3.7. Theorem. Let $\Omega$ be a compact domain in $\mathcal{R}^{2}$ and let $f^{0}$ be a function in the space $C_{s}^{k, 2+\alpha}(\Omega)$, for some nonnegative integer $k$ and some number $\alpha$ in $0<\alpha<1$. Assume that $f^{0}=0$ at $\partial \Omega, f^{0}>0$ in the interior of $\Omega$ so that

$$
\left|D f^{0}(x, y)\right|+f^{0}(x, y) \geq c>0 \quad \forall(x, y) \in \Omega
$$

Then, there exists a number $\tau_{k}$, for which the initial free-boundary problem

$$
\begin{cases}f_{t}=f \Delta f+r|D f|^{2}, & (x, y, t) \in \Omega_{t} \times\left[0, \tau_{k}\right], \\ f(x, y, 0)=f^{0}, & (x, y) \in \Omega\end{cases}
$$

with $r>0$, admits a solution $f$ in $C_{s}^{k, 2+\alpha}\left(\Omega_{t} \times\left[0, \tau_{k}\right]\right)$.

Proof. As we have seen in Theorem III.3.2 the coordinate change $\Phi$ in (III.3.1) converts the given initial free-boundary problem to the initial value problem with fixed boundary

$$
\begin{cases}M w=0, & (u, v, t) \in \mathcal{D} \times[0, T] \\ w(u, v, 0)=w^{0}, & (u, v) \in \mathcal{D}\end{cases}
$$

with

$$
M w=w_{t}-\vartheta F^{i j}(t, u, v, w, D w) w_{i j}-G(t, u, v, w, D w)
$$

and $w^{0} \in C_{s}^{k, 2+\alpha}(\mathcal{D})$. However we have shown that if $T \leq \tau_{k}$, with $\tau_{k}$ sufficiently small depending on $k$ and $c$, the operator $M$ satisfies all the hypotheses of Theorem 2.1 in Part II. Therefore, by this theorem the initial value problem for the equation $M w=0$ admits a solution $w \in C_{s}^{k, 2+\alpha}\left(\mathcal{D} \times\left[0, \tau_{k}\right]\right)$. If we express the function $w$ in the old coordinates we obtain a solution $z=f(x, y, t)$ of the given initial free-boundary problem which belongs to the space $C_{s}^{k, 2+\alpha}\left(\Omega \times\left[0, \tau_{k}\right]\right)$. The computations are the same as in the proof of Theorem III.3.2.

III.4. $C^{\infty}$-regularity. In this final section we will give the proof of Theorem III.1.1. It will follow from the next regularity result.

III.4.1. Theorem. Assume that for some $T>0$ and some number $\alpha$ in $0<\alpha<$ $1, f \in C_{s}^{2+\alpha}\left(\Omega_{t} \times[0, T]\right)$ is a solution of the free-boundary problem

$$
\begin{cases}f_{t}=f \Delta f+r|D f|^{2}, & (x, y, t) \in \Omega_{t} \times[0, T] \\ f(x, y, 0)=f^{0}, & (x, y) \in \Omega\end{cases}
$$

so that

$$
|D f(x, y, t)|+f(x, y, t) \geq c>0 \quad \forall(x, y, t) \in \Omega_{t} \times[0, T]
$$

Then, for any positive integer $k, f \in C_{s}^{k, 2+\alpha}\left(\Omega_{t} \times(0, T]\right)$ and for any $\tau$ in $0<\tau<T$ we have

$$
\|f\|_{C_{s}^{k, 2+\alpha}\left(\Omega_{t} \times[\tau, T]\right)} \leq C_{k}\left(\tau,\left\|f^{0}\right\|_{C_{s}^{2+\alpha}(\Omega)}\right) .
$$

Proof. We begin with the proof of the theorem for $k=1$. Choose a family of smooth regularizations $f_{\epsilon}^{0}$ of the initial data $f^{0}$, approximating $f^{0}$, so that each $f_{\epsilon}^{0}$ is strictly positive in the interior of a compact domain $\Omega_{\epsilon}$, with $f_{\epsilon}=0$ at $\partial \Omega_{\epsilon}$ and

$$
\left|D f_{\epsilon}^{0}(x, y)\right|+f_{\epsilon}^{0}(x, y, t) \geq c>0 \quad \forall(x, y) \in \Omega_{\epsilon} .
$$


It follows from the results in Section 11, Part I, that we can choose the functions $f_{\epsilon}^{0}$ so that

$$
\left\|f_{\epsilon}^{0}\right\|_{C_{s}^{2+\alpha}\left(\Omega_{\epsilon}\right)} \leq C\left\|f^{0}\right\|_{C_{s}^{2+\alpha}(\Omega)}
$$

with the constant $C$ independent of $\epsilon$ and $f_{\epsilon}^{0} \rightarrow f^{0}$ as $\epsilon \rightarrow 0$ in the sense that

$$
D_{x}^{i} D_{y}^{j} D_{t}^{k} f_{\epsilon} \rightarrow D_{x}^{i} D_{y}^{j} D_{t}^{k} f
$$

if $j+j+k \leq 1$ and

$$
d_{\epsilon} D_{x}^{i} D_{y}^{j} f_{\epsilon} \rightarrow d_{\epsilon} D_{x}^{i} D_{y}^{j} f
$$

if $j+j=2$, with $d_{\epsilon}$ denoting the distance to the boundary of $\Omega_{\epsilon}$.

Denote by $f_{\epsilon}$ the solution to the free boundary problem

$$
\begin{cases}f_{t}=f \Delta f+r|D f|^{2}, & (x, y, t) \in \Omega_{\epsilon, t} \times\left[0, \tau_{1}\right], \\ f(x, y, 0)=f_{\epsilon}^{0}, & (x, y) \in \Omega_{\epsilon},\end{cases}
$$

as constructed in Theorem III.3.7. It follows from the same theorem that there exists a number $\tau_{1}$ in $0<\tau_{1} \leq T$ which is independent of $\epsilon$ such that $f_{\epsilon} \in$ $C_{s}^{1,2+\alpha}\left(\Omega_{\epsilon, t} \times\left[0, \tau_{1}\right]\right)$. Moreover,

$$
\left\|f_{\epsilon}\right\|_{C_{s}^{1,2+\alpha}\left(\Omega_{\epsilon, t} \times\left[0, \tau_{1}\right]\right)} \leq C\left(\left\|f_{\epsilon}^{0}\right\|_{C_{s}^{1,2+\alpha}\left(\Omega_{\epsilon}\right)}\right) .
$$

On the other hand, since $\left\|f_{\epsilon}^{0}\right\|_{C_{s}^{2+\alpha}\left(\Omega_{\epsilon}\right)} \leq C\left\|f^{0}\right\|_{C_{s}^{2+\alpha}(\Omega)}$ the result in Theorem III.3.7 implies that

$$
\left\|f_{\epsilon}\right\|_{C_{s}^{2+\alpha}\left(\Omega_{\epsilon, t} \times[0, T]\right)} \leq C\left(\left\|f^{0}\right\|_{C_{s}^{2+\alpha}(\Omega)}\right)
$$

which is a bound that is independent of $\epsilon$. Therefore, it is easy to see that passing to a subsequence (still denoted by $f_{\epsilon}$ ) the sequence $f_{\epsilon}$ will converge to $f$, being the unique weak solution of the equation

$$
f_{t}=f \Delta f+r|D f|^{2} \quad \text { on } \mathcal{R}^{2} \times[0, T]
$$

with initial data $f^{0}$.

Our first goal is to show that for $\tau$ in $0<\tau<\tau_{1}$ we have

$$
\left\|f_{\epsilon}\right\|_{C_{s}^{1,2+\alpha}\left(\Omega_{\epsilon, t} \times\left[\tau, \tau_{1}\right]\right)} \leq C\left(\tau,\left\|f^{0}\right\|_{C_{s}^{2+\alpha}(\Omega)}\right)
$$

with $C=C\left(\left\|f^{0}\right\|_{C_{s}^{2+\alpha}(\Omega)}\right)$ independent of $\epsilon$. For this purpose we will use the local change of variables introduced in Section 2 of Part III.

For $\epsilon>0$ and $\tau$ in $0<\tau<\tau_{1}$ fixed, we pick a point $P_{0}=\left(x_{0}, y_{0}, t_{0}\right)$ at the free boundary $\partial \Omega_{\epsilon, t} \times\left(\tau, \tau_{1}\right)$. We can assume with no loss of generality (by rotating the coordinates) that

$$
\left(f_{\epsilon}\right)_{x}\left(P_{0}\right)>0, \quad\left(f_{\epsilon}\right)_{y}\left(P_{0}\right)=0, \quad\left(f_{\epsilon}\right)_{t}\left(P_{0}\right)=r\left(f_{\epsilon}\right)_{x}^{2}\left(P_{0}\right)>0 .
$$

Then, as we showed in Section 2, locally around the point $P_{0}$, we can solve the equation $z=f_{\epsilon}(x, y, t)$ with respect to $x$ yielding to a function $x=h_{\epsilon}(z, y, t)$ defined on a small box

$$
\mathcal{B}_{\eta}=\left\{0 \leq z \leq \eta,\left|y-y_{0}\right| \leq \eta,-\eta \leq t-t_{0} \leq 0\right\} .
$$

After a moment of thought we realize that the number $\eta$ can be taken to be independent of the particular point $P_{0}$ on the free boundary $\Omega_{\epsilon, t} \times[0, T]$ and independent $\epsilon$. This is because the norms $\left\|f_{\epsilon}\right\|_{C_{s}^{2+\alpha}\left(\Omega_{\epsilon} \times[0, T]\right)}$ are uniformly bounded. 
To simplify the notation we will denote for the moment the function $h_{\epsilon}$ by $h$. We have computed in Section 2 that on the set $\mathcal{B}_{\eta}$ we have

$$
h_{z}=\frac{1}{f_{x}}, \quad h_{y}=-\frac{f_{y}}{h_{z}}
$$

and therefore

$$
h_{z}(z, y, t)>0, \quad h_{y}(x, y, t) \sim 0
$$

if $\eta$ is sufficiently small. Moreover, each $h_{\epsilon}$ satisfies the equation

$$
h_{t}-\left\{z\left(\frac{1+h_{y}^{2}}{h_{z}^{2}} h_{z z}-\frac{2 h_{y}}{h_{z}} h_{z y}+h_{y y}\right)-r \frac{1+h_{y}^{2}}{h_{z}}\right\}=0
$$

for all $(z, y, t) \in \mathcal{B}_{\eta}$.

Our goal is to establish a $C_{s}^{1,2+\alpha}$ bound for $h=h_{\epsilon}$ in the box $\mathcal{B}_{\eta}$ which is independent of $\epsilon$. To do so we will compute, by differentiating the above equation, the evolution of the derivatives $h_{z}, h_{y}$ and $h_{t}$ on $\mathcal{B}_{\eta}$ and then we will apply the local Shauder estimates in Theorem II.1.2.

We will first establish this bound for $h_{y}$. The reader can easily chack that $w=h_{y}$, when restricted on the box $\mathcal{B}_{\eta}$, satisfies the equation $M_{\epsilon, y}(w)=0$ where

$$
\begin{aligned}
M_{\epsilon, y}(w)=w_{t}- & \left\{z\left(\frac{1+h_{y}^{2}}{h_{z}^{2}} w_{z z}-\frac{2 h_{y}}{h_{z}} w_{z y}+w_{y y}\right)\right. \\
+ & \left(\frac{r\left(1+h_{y}^{2}\right)}{h_{z}^{2}}-\frac{2 z\left(1+h_{y}^{2}\right) h_{z z}}{h_{z}^{3}}+\frac{2 z h_{y} h_{z y}}{h_{z}^{2}}\right) w_{z} \\
& \left.+\left(\frac{-2 r h_{y}}{h_{z}}+\frac{2 z h_{y} h_{z z}}{h_{z}^{2}}-\frac{2 z h_{z y}}{h_{z}}\right) w_{y}\right\} .
\end{aligned}
$$

After a moment of thought we see that all the coefficients of the operator $M_{\epsilon, y}$ belong to the space $C_{s}^{\alpha}\left(\mathcal{B}_{\eta}\right)$ with norms uniformly bounded by a constant which depends only on $\left\|f^{0}\right\|_{C_{s}^{2+\alpha}(\Omega)}$. Moreover,

$$
\frac{r\left(1+h_{y}^{2}\right)}{h_{z}^{2}}-\frac{2 z\left(1+h_{y}^{2}\right) h_{z z}}{h_{z}^{3}}+\frac{2 z h_{y} h_{z y}}{h_{z}^{2}} \geq \frac{r}{2}>0
$$

if $\eta$ is sufficiently small, independently of $\epsilon$. Therefore, the operator $M_{\epsilon, y}$ satisfies all the hypotheses of Theorem 1.3 in Part II and in conclusion the following estimate holds (for $h=h_{\epsilon}$ ):

$$
\left\|h_{y}\right\|_{C_{s}^{2+\alpha}\left(\mathcal{B}_{\eta / 2}\right)} \leq C\left(\left\|f^{0}\right\|_{C_{s}^{2+\alpha}(\Omega)}\right) .
$$

The same estimate can be shown to hold true for the time derivative $w=h_{t}$ which satisfies the same equation, namely $M_{\epsilon, t}(w)=0$, with

$$
\begin{aligned}
M_{\epsilon, t}(w)=w_{t}- & \left\{z\left(\frac{1+h_{y}^{2}}{h_{z}^{2}} w_{z z}-\frac{2 h_{y}}{h_{z}} w_{z y}+w_{y y}\right)\right. \\
+ & +\left(\frac{r\left(1+h_{y}^{2}\right)}{h_{z}^{2}}-\frac{2 z\left(1+h_{y}^{2}\right) h_{z z}}{h_{z}^{3}}+\frac{2 z h_{y} h_{z y}}{h_{z}^{2}}\right) w_{z} \\
& \left.+\left(\frac{-2 r h_{y}}{h_{z}}+\frac{2 z h_{y} h_{z z}}{h_{z}^{2}}-\frac{2 z h_{z y}}{h_{z}}\right) w_{y}\right\} .
\end{aligned}
$$


Finally, the derivative $w=h_{z}$ satisfies the equation $M_{\epsilon, z}=0$ where $M_{\epsilon, z}$ is the operator

$$
\begin{aligned}
M_{\epsilon, z}(w)=w_{t}-\{ & \left(\frac{1+h_{y}^{2}}{h_{z}^{2}} w_{z z}-\frac{2 h_{y}}{h_{z}} w_{z y}+w_{y y}\right) \\
+ & \left(\frac{(r+1)\left(1+h_{y}^{2}\right)}{h_{z}^{2}}-\frac{2 z\left(1+h_{y}^{2}\right) h_{z z}}{h_{z}^{3}}\right) w_{z} \\
& \left.\quad+\left(-\frac{2(r+1) h_{y}}{h_{z}}+\frac{4 z h_{y} h_{z z}}{h_{z}^{2}}-\frac{2 z h_{z y}}{h_{z}}\right) w_{y}+h_{y y}\right\} .
\end{aligned}
$$

Since $h \in C_{s}^{1,2+\alpha}\left(\mathcal{B}_{\eta / 2}\right)$, the function $h_{y y}$ belongs in $C_{s}^{1,2+\alpha}\left(\mathcal{B}_{\eta / 2}\right)$. Hence, the desired estimate follows as above from Theorem 1.3 in Part II.

We conclude from the above that $h=h_{\epsilon}$ satisfies

$$
\left\|h_{\epsilon}\right\|_{C_{s}^{1,2+\alpha}\left(\mathcal{B}_{\eta / 2}\right)} \leq C\left(\left\|f^{0}\right\|_{C_{s}^{2+\alpha}(\Omega)}\right) .
$$

We can now go back to the original coordinates. The transformation of first and second derivatives via the coordinate change, as computed in Section 2, and our estimates above show that if for some number $\tau$ in $0<\tau<\tau_{1}, \mathcal{A}_{\eta, \epsilon}^{\tau}$ denotes the set

$$
\mathcal{A}_{\eta, \epsilon}^{\tau}=\left\{(x, y, t) \in \Omega_{\epsilon} \times\left[\tau, \tau_{1}\right]: f_{\epsilon}(x, y, t) \leq \eta\right\},
$$

then we have

$$
\left\|f_{\epsilon}\right\|_{C_{s}^{1,2+\alpha}\left(\mathcal{A}_{\eta, \epsilon}^{\tau}\right)} \leq C\left(\tau,\left\|f^{0}\right\|_{C_{s}^{2+\alpha}(\Omega)}\right) .
$$

The same estimate holds true on the interior set

$$
\Gamma_{\eta, \epsilon}^{\tau}=\left(\Omega_{\epsilon, t} \times\left[\tau, \tau_{1}\right]\right) \backslash \mathcal{A}_{\eta, \epsilon}^{\tau}
$$

as it follows from the standard regularity theory of nondegenerate parabolic equations. Therefore we conclude that

$$
\left\|f_{\epsilon}\right\|_{C_{s}^{1,2+\alpha}\left(\Omega_{\epsilon} \times\left[\tau, \tau_{1}\right]\right)} \leq C\left(\tau,\left\|f^{0}\right\|_{C_{s}^{2+\alpha}(\Omega)}\right)
$$

for all $\tau$ in $0<\tau<\tau_{1}$, as desired.

Next we extend each solution $f_{\epsilon}$ so that $f_{\epsilon}(x, t)=0$ for all $x \in \mathcal{R}^{2} \backslash \Omega_{\epsilon, t}$, $0 \leq t \leq \tau_{1}$, so that each $f_{\epsilon}$ becomes a weak solution of the Cauchy problem

$$
\begin{cases}f_{t}=f \Delta f+r|D f|^{2}, & (x, y, t) \in \mathcal{R}^{2} \times\left[0, \tau_{1}\right], \\ f(x, y, 0)=f_{\epsilon}^{0}, & (x, y) \in \mathcal{R}^{2} .\end{cases}
$$

The sequence of solutions $\left\{f_{\epsilon}\right\}$ is equicontinuous and uniformly bounded on $\mathcal{R}^{2} \times$ $\left[0, \tau_{1}\right]$, since

$$
\left\|f_{\epsilon}\right\|_{C_{s}^{2+\alpha}\left(\Omega_{\epsilon} \times\left[0, \tau_{1}\right]\right)} \leq C\left(\left\|f^{0}\right\|_{C_{s}^{2+\alpha}(\Omega)}\right) .
$$

Therefore there exists a subsequence, still denoted by $f_{\epsilon}$ which converges, as $\epsilon \rightarrow 0$, to the given solution $f$ (extended to be equal to zero outside $\Omega_{t} \times\left[0, \tau_{1}\right]$ ). The convergence is uniform on compact subsets of $\mathcal{R}^{2} \times\left[0, \tau_{1}\right]$. In particular

$$
\operatorname{dist}\left(\partial \Omega_{\epsilon, t}, \partial \Omega_{t}\right) \rightarrow 0 \quad \text { as } \epsilon \rightarrow 0
$$

uniformly on $t$ in the interval $0 \leq t \leq \tau_{1}$. However, when restricted on $\Omega_{t} \times\left[\tau, \tau_{1}\right]$, for any $\tau$ in $0<\tau<\tau_{1}$ the convergence is much stronger. Indeed, it follows from the uniform bound

$$
\left\|f_{\epsilon}\right\|_{C_{s}^{1,2+\alpha}\left(\Omega_{\epsilon} \times\left[\tau, \tau_{1}\right]\right)} \leq C\left(\tau,\left\|f^{0}\right\|_{C_{s}^{2+\alpha}(\Omega)}\right)
$$


that there exists a subsequence of $\left\{f_{\epsilon}\right\}$, still denoted by $f_{\epsilon}$, such that for any $(x, t) \in \Omega \times\left[\tau, \tau_{1}\right]$ we have

$$
D_{x}^{i} D_{y}^{j} D_{t}^{k} f_{\epsilon}(x, y, t) \rightarrow D_{x}^{i} D_{y}^{j} D_{t}^{k} f(x, y, t)
$$

if $i+j+k \leq 2$ and

$$
d^{\epsilon} D_{x}^{i} D_{y}^{j} f_{\epsilon}(x, y, t) \rightarrow d D_{x}^{i} D_{y}^{j} f(x, y, t)
$$

for $i+j=3$, as $\epsilon$ tends to zero, where $d^{\epsilon}$ denotes the distance to the free boundary $\Omega_{\epsilon} \times\left[\tau, \tau_{1}\right]$. It is now easy to check that the solution $f$ belongs to the space $C_{s}^{1,2+\alpha}\left(\Omega_{\epsilon} \times\left(0, \tau_{1}\right]\right)$ and for all $\tau$ in $0<\tau<\tau_{1}$ satisfies the estimate

$$
\|f\|_{C_{s}^{1,2+\alpha}\left(\Omega_{t} \times\left[\tau, \tau_{1}\right]\right)} \leq C\left(\tau,\left\|f^{0}\right\|_{C_{s}^{2+\alpha}(\Omega)}\right) .
$$

Finally, assume that $N \tau_{1}<T \leq(N+1) \tau_{1}$, for some nonnegative integer $N$. Since

$$
\|f\|_{C_{s}^{2+\alpha}\left(\Omega_{t} \times[0, T]\right)} \leq C\left(\left\|f^{0}\right\|_{C_{s}^{2+\alpha}(\Omega)}\right)
$$

we can repeat the above estimate $N+1$ times to finally conclude that

$$
\|f\|_{C_{s}^{1,2+\alpha}\left(\Omega_{t} \times[\tau, T]\right)} \leq C\left(\tau,\left\|f^{0}\right\|_{C_{s}^{2+\alpha}(\Omega)}\right)
$$

for all $0<\tau<T$, as desired.

This proves the Theorem in the case of $k=1$. The case of a general $k$ can be shown via induction by differentiating the local equation

$$
h_{t}-z\left(\frac{1+h_{y}^{2}}{h_{z}^{2}} h_{z z}-\frac{2 h_{y}}{h_{z}} h_{z y}+h_{y y}\right)+r \frac{1+h_{y}^{2}}{h_{z}}=0
$$

$k$ times and using once more the local Schauder estimate, Theorem II.1.3.

We finish with the proof of Theorem III.1.1.

Proof of Theorem III.1.1. From Theorem III.3.7, there exists a solution $f \in$ $C_{s}^{2+\alpha}\left(\Omega_{t} \times[0, T]\right)$ of the free boundary problem

$$
\begin{cases}f_{t}=f \Delta f+r|D f|^{2}, & (x, y, t) \in \Omega_{t} \times[0, T] \\ f(x, y, 0)=f^{0}, & (x, y) \in \Omega\end{cases}
$$

for some number $T>0$. Moreover, since $D f^{0} \neq 0$ at $\partial \Omega$, we can choose the number $T$ so that

$$
|D f(x, y, t)|+f(x, y, t) \geq c \quad \forall(x, y, t) \in \Omega_{t} \times[0, T]
$$

for some $c>0$. But then, it follows from Theorem III.4.1 that

$$
f \in C_{s}^{k, 2+\alpha}\left(\Omega_{t} \times[0, T]\right)
$$

for all positive integers $k$. We conclude that $f \in C^{\infty}\left(\Omega_{t} \times[0, T]\right)$, as desired. In particular the free-boundary is smooth. 


\section{REFERENCES}

[A] S. Angenent, Analyticity of the interface of the porous media equation after the waiting time, Proc. Amer. Math. Soc. 102 (1988), N2, 329-336 MR 89f:35103

[A1] D.G. Aronson, Regularity properties of flows through porous media, SIAM J. Appl. Math. 17 (1969), 461-467. MR 40:571

[A2] D.G. Aronson, Regularity properties of flows through porous media: A counterexample, SIAM J. Appl. Math. 19 (1970), 299-307. MR 42:683

[A3] D.G. Aronson, Regularity properties of flows through porous media: The interface, Arch. Rational Mech. Anal. 37 (1970), 1-10. MR 41:656

[ACV] D.G. Aronson, L.A. Caffarelli, J.L. Vázquez, Interfaces with a corner-point in onedimensional porous medium flow, Comm. Pure and Appl. Math. 38 (1985), 375-404. MR 86h:35070

[AV] D. Aronson, J.L. Vázquez, Eventual regularity and concavity for flows in one dimensional porous media, Arch. Rational Mech. Anal. 99 (1987), 329-348. MR 89d:35081

[C] L. Caffarelli, Interior a priori estimates for solutions of fully non-linear equations, Ann. of Math. 130 (1989), 189-213. MR 90i:35046

[CF1] L.A. Caffarelli and A. Friedman, Regularity of the free boundary for the one-dimensional flow of gas in a porous medium, Amer. J. Math. 101 (1979), 1193-1218. MR 80k:76072

[CF2] L.A. Caffarelli and A. Friedman, Regularity of the free boundary of a gas flow in an n-dimensional porous medium, Ind. Univ. Math. J. 29 (1980), 361-391. MR 82a:35096

[CVW] L.A. Caffarelli, J.L. Vázquez, N.I. Wolanski, Lipschitz continuity of solutions and interfaces of the n-dimensional porous medium equation, Ind. Univ. Math. J. 36 (1987), 373-401. MR 88k:35221

[CW] L.A. Caffarelli, N.I. Wolanski, $C^{1, \alpha}$ regularity of the free boundary for the $n$-dimensional porous media equation, Comm. Pure and Appl. Math. 43 (1990), 885-902. MR 91h:35332

[HK] K. Höllig, H.O. Kreiss, $C^{\infty}$ regularity for the porous medium equation, Univ. of Winsconsin Madison, Computer Scienced Dept., Technical report \# 600.

$[\mathrm{K}] \quad$ B. Knerr, The porous medium equation in one dimension, Trans. Amer. Math. Soc. 234 (1977), 381-415. MR 58:11917

[KN] J.J. Kohn, L. Nirenberg, Degenerate elliptic-parabolic equations of second order, Comm. Pure and Appl. Math. 20 (1967), 797-872. MR 38:2437

[S1] M. Safonov On the classical solution of Bellman's elliptic equations, Dokl. Akad. Nauk SSSR 278 (1984), N4, 810-813 = Soviet Math. Dokl. 30 (1984), N2, 482-485 MR 86f: 35081

[S2] M. Safonov On the classical solution of nonlinear elliptic equations of second order, Izv. Akad. Nauk SSSR. Ser. Mat. 52 (1988), N6, 1272-1287 = Math. USSR Izvestiya, 33 (1989), N3, 597-612 MR 90d:35104

[W1] L. Wang On the regularity theory of fully nonlinear parabolic equations I, Comm. Pure and Appl. Math., 45, 1992, N1, 27-76. MR 92m:35126

[W2] L.Wang On the regularity theory of fully nonlinear parabolic equations II, Comm. Pure and Appl. Math. 45, 1992, N2, 141-178. MR 92m:35127

Department of Mathematics, University of California, Irvine, California 92697-3875

E-mail address: pdaskalo@math.uci.edu

Department of Mathematics, University of California at San Diego, La Jolla, CalIFORNIA 92093-0001 